\title{
Scale-Dependent Priors for Variance Parameters in Structured Additive Distributional Regression Supplement
}

\author{
Nadja Klein, Thomas Kneib \\ Chair of Statistics \\ Georg-August-University Göttingen
}

\begin{abstract}
This is a supplement to the paper 'Scale-Dependent Priors for Variance Parameters in Structured Additive Distributional Regression' in which

- the proofs of Theorems 1 to 3 as well as further technical details are documented in Section $\mathrm{A}$,

- empirical experiments are presented in more detail in Sections B and G.

- further theoretical and implementational details, as well as computations on the approximate uniform prior, priors on the distance scale and IWLS proposals are presented in Sections C to $\mathrm{F}$, and

- additional figures to the case studies of Sections 6.1 and 6.2 of the main paper are contained in Sections $\mathrm{H}$ and $\mathrm{I}$.
\end{abstract}




\section{A Proofs and Technical Details}

\section{A.1 Proof of Theorem 1}

Proof. The strategy is to start with $\tau_{b}^{2}>0$ small and to derive required quantities before finally proofing the theorem by considering the limit $\tau_{b}^{2} \rightarrow 0$.

The KLD as defined in (5) of the $\kappa=\operatorname{rk}(\boldsymbol{K})$-dimensional stochastic part between the base model and the flexible one is given by

$$
\mathrm{KLD}=\frac{\kappa}{2} \frac{\tau^{2}}{\tau_{b}^{2}}\left(1+\frac{\tau_{b}^{2}}{\tau^{2}} \log \left(\frac{\tau_{b}^{2}}{\tau^{2}}\right)-\frac{\tau_{b}^{2}}{\tau^{2}}\right) .
$$

From the assumption of constant rate penalisation, we therefore obtain the prior on the original space as

$$
\begin{aligned}
& p\left(\tau^{2}\right)=\lambda \exp \left(-\lambda d\left(\tau^{2}\right)\right)\left|\frac{\partial d\left(\tau^{2}\right)}{\partial \tau^{2}}\right| \text { with } d\left(\tau^{2}\right)=\sqrt{2 \mathrm{KLD}} \text { and } \\
& -\lambda d\left(\tau^{2}\right)=-\lambda \sqrt{\frac{\kappa}{\tau_{b}^{2}}}\left(\tau^{2}\right)^{1 / 2} \sqrt{1+\frac{\tau_{b}^{2}}{\tau^{2}} \log \left(\frac{\tau_{b}^{2}}{\tau^{2}}\right)-\frac{\tau_{b}^{2}}{\tau^{2}}} \\
& \lambda\left|\frac{\partial d\left(\tau^{2}\right)}{\partial \tau^{2}}\right|=\frac{1}{2} \lambda \sqrt{\frac{\kappa}{\tau_{b}^{2}}}\left(\frac{1}{\tau^{2}}-\frac{\tau_{b}^{2}}{\tau^{3}}\right)\left(1+\frac{\tau_{b}^{2}}{\tau^{2}} \log \left(\frac{\tau_{b}^{2}}{\tau^{2}}\right)-\frac{\tau_{b}^{2}}{\tau^{2}}\right)^{-1 / 2} .
\end{aligned}
$$

Set $\theta^{-1 / 2}=\lambda \sqrt{\frac{\kappa}{\tau_{b}^{2}}}$ and choose $\lambda$ such that $\theta^{-1 / 2}$ is kept constant for $\tau_{b}^{2} \rightarrow 0$. Then, in the limit, i.e. $\tau_{b}^{2} \rightarrow 0$ we obtain

$$
\begin{gathered}
-\lambda d\left(\tau^{2}\right) \stackrel{\tau_{b}^{2} \rightarrow 0}{\rightarrow}-\left(\frac{\tau^{2}}{\theta}\right)^{1 / 2} \\
\lambda\left|\frac{\partial d\left(\tau^{2}\right)}{\partial \tau^{2}}\right| \stackrel{\tau_{b}^{2} \rightarrow 0}{\rightarrow} \frac{1}{2}\left(\frac{1}{\theta}\right)^{1 / 2}\left(\tau^{2}\right)^{-1 / 2}
\end{gathered}
$$

due to $\frac{\tau_{b}^{2}}{\tau^{2}} \log \left(\frac{\tau_{b}^{2}}{\tau^{2}}\right) \stackrel{\tau_{b}^{2} \rightarrow 0}{\rightarrow} 0$ and $\frac{\tau_{b}^{2}}{\tau^{2}} \stackrel{\tau_{b}^{2} \rightarrow 0}{\rightarrow} 0$ and hence

$$
p\left(\tau^{2}\right)=\lambda \exp \left(-\lambda d\left(\tau^{2}\right)\right)\left|\frac{\partial d\left(\tau^{2}\right)}{\partial \tau^{2}}\right|=\frac{1}{2}\left(\frac{1}{\theta}\right)^{1 / 2}\left(\tau^{2}\right)^{-1 / 2} \exp \left(-\left(\frac{\tau^{2}}{\theta}\right)^{1 / 2}\right)
$$

which completes the proof.

\section{A.2 Preliminary Results For Proofs of Theorem 2 and 3}

Proposition 1. Let $\operatorname{rk}(\boldsymbol{U})=r$ and let the remaining definitions be as in Section 3.2. Then the following statements are equivalent:

(i) $\operatorname{rk}\left(\boldsymbol{V}^{\prime} \boldsymbol{R}_{1} \boldsymbol{V}\right)=t$.

(ii) $\operatorname{rk}\left(\begin{array}{ll}\boldsymbol{U}^{\prime} \boldsymbol{U} & \boldsymbol{U}^{\prime} \boldsymbol{V} \\ \boldsymbol{V}^{\prime} \boldsymbol{U} & \boldsymbol{V}^{\prime} \boldsymbol{V}\end{array}\right)=r+t$

(iii) $\operatorname{rk}(\boldsymbol{U}, \boldsymbol{V})=r+t$. 
Proof.

(ii) $\Leftrightarrow($ iii $)$ Due to $\operatorname{rk}(\boldsymbol{M})=\operatorname{rk}\left(\boldsymbol{M}^{\prime} \boldsymbol{M}\right)$ for a real matrix $\boldsymbol{M}$, the claim follows directly by setting $\boldsymbol{M}=(\boldsymbol{U}, \boldsymbol{V})$ and thus

$$
M^{\prime} M=\left(\begin{array}{ll}
U^{\prime} U & U^{\prime} \boldsymbol{V} \\
\boldsymbol{V}^{\prime} \boldsymbol{U} & \boldsymbol{V}^{\prime} \boldsymbol{V}
\end{array}\right)
$$

(i) $\Leftrightarrow($ iii) Let $\boldsymbol{W}=\mathcal{M}(\boldsymbol{U}, \boldsymbol{V})$ be the vector space generated from $(\boldsymbol{U}, \boldsymbol{V})$ with subspace $\tilde{\boldsymbol{W}} \subset \boldsymbol{W}$. Then we have $\boldsymbol{W}=\tilde{\boldsymbol{W}}+\tilde{\boldsymbol{W}}^{\perp}$ with $\tilde{\boldsymbol{W}}^{\perp}$ being the orthogonal complement of $\tilde{\boldsymbol{W}}$. Let $\tilde{\boldsymbol{W}}=\mathcal{M}(\boldsymbol{U})$ (and hence $\operatorname{dim}(\tilde{\boldsymbol{W}})=r$ ), then $\boldsymbol{P}=\boldsymbol{U}\left(\boldsymbol{U}^{\prime} \boldsymbol{U}\right)^{-1} \boldsymbol{U}^{\prime}$ is the projection onto $\tilde{\boldsymbol{W}}$ and $\boldsymbol{R}_{1}=\boldsymbol{I}_{n}-\boldsymbol{P}$ is the projection onto $\tilde{\boldsymbol{W}}^{\perp}$. Consequently,

$$
\begin{aligned}
\mathcal{M}(\boldsymbol{U}, \boldsymbol{V}) & =\mathcal{M}(\boldsymbol{P}(\boldsymbol{U}, \boldsymbol{V}))+\mathcal{M}\left(\boldsymbol{R}_{1}(\boldsymbol{U}, \boldsymbol{V})\right) \\
& =\mathcal{M}(\boldsymbol{P} \boldsymbol{U}, \boldsymbol{P} \boldsymbol{V})+\mathcal{M}\left(\boldsymbol{R}_{1} \boldsymbol{U}, \boldsymbol{R}_{1} \boldsymbol{V}\right) \\
& =\mathcal{M}(\boldsymbol{U}, \boldsymbol{P} \boldsymbol{V})+\mathcal{M}\left(\mathbf{0}, \boldsymbol{R}_{1} \boldsymbol{V}\right) \\
& =\mathcal{M}(\boldsymbol{U})+\mathcal{M}\left(\boldsymbol{R}_{1} \boldsymbol{V}\right)
\end{aligned}
$$

and hence

$$
\operatorname{dim}(\mathcal{M}(\boldsymbol{U}, \boldsymbol{V}))=\operatorname{dim}(\mathcal{M}(\boldsymbol{U}))+\operatorname{dim}\left(\mathcal{M}\left(\boldsymbol{R}_{1} \boldsymbol{V}\right)\right) .
$$

Finally, the claim follows, since

$$
\begin{gathered}
" \Leftarrow " \operatorname{rk}(\boldsymbol{U}, \boldsymbol{V})=r+t \text { and } \operatorname{rk}(\boldsymbol{U})=r \\
\Rightarrow \operatorname{rk}\left(\boldsymbol{R}_{1} \boldsymbol{V}\right)=t . \\
" \Rightarrow " \operatorname{rk}\left(\boldsymbol{R}_{1}, \boldsymbol{V}\right)=t \text { and } \operatorname{rk}(\boldsymbol{U})=r \\
\Rightarrow \operatorname{rk}(\boldsymbol{U}, \boldsymbol{V})=r+t .
\end{gathered}
$$

Lemma 2. Assume that $\beta>0$ is known and define

$$
\mathcal{I} \equiv \int_{0}^{\infty} \exp \left(-\sqrt{\frac{u}{\beta}}\right) \frac{1}{\sqrt{u}}\left(\frac{1}{1+u}\right)^{\gamma} \mathrm{d} u .
$$

2.1 Let $\mathrm{B}(x, y)$ be the beta function of $x, y>0$. If $\gamma>\frac{1}{2}$ an upper bound for $\mathcal{I}$ is given by

$$
\mathrm{B}(1 / 2, \gamma-1 / 2)
$$

2.2 For all $\gamma \in \mathbb{R}$ a lower bound for $\mathcal{I}$ is given by

$$
\mathcal{I} \geq \begin{cases}\exp \left(-\beta^{-1 / 2}\right) \log (2) & \text { if } \gamma=\frac{1}{2} \\ \exp \left(-\beta^{-1 / 2}\right)\left(-\gamma+\frac{1}{2}\right)^{-1}\left[2^{-\gamma+1 / 2}-1\right] & \text { else. }\end{cases}
$$

Proof.

2.1 Assume that $\gamma>\frac{1}{2}$ holds. Then,

$$
\begin{aligned}
\mathcal{I} & \leq \int_{0}^{\infty} \frac{1}{\sqrt{u}}\left(\frac{1}{u+1}\right)^{\gamma} \mathrm{d} u \\
& =\mathrm{B}(1 / 2, \gamma-1 / 2) .
\end{aligned}
$$


2.2 2

$$
\begin{aligned}
\mathcal{I} & \geq \int_{0}^{1} \exp \left(-\sqrt{\frac{u}{\beta}}\right) \frac{1}{\sqrt{u}}\left(\frac{1}{u+1}\right)^{\gamma} \mathrm{d} u \\
& \geq \int_{0}^{1} \exp \left(-\beta^{-1 / 2}\right)\left(\frac{1}{u+1}\right)^{\gamma+1 / 2} \mathrm{~d} u \\
& = \begin{cases}\exp \left(-\beta^{-1 / 2}\right) \log (2) & \text { if } \gamma=\frac{1}{2} \\
\exp \left(-\beta^{-1 / 2}\right)\left(-\gamma+\frac{1}{2}\right)^{-1}\left[2^{-\gamma+1 / 2}-1\right] & \text { else. }\end{cases}
\end{aligned}
$$

\section{A.3 Proof of Theorem 2}

Define in analogy to Sun et al. (2001)

$$
\begin{aligned}
\boldsymbol{R}_{2} & =\frac{1}{\tau_{\varepsilon}^{2}} \boldsymbol{V}^{\prime} \boldsymbol{R}_{1} \boldsymbol{V}+\boldsymbol{T}^{-1}, \text { with } \boldsymbol{T}=\operatorname{diag}\left(\tau_{1}^{2} \boldsymbol{I}_{\kappa_{1}}, \ldots, \tau_{J}^{2} \boldsymbol{I}_{\kappa_{J}}\right) \\
\boldsymbol{R}_{3} & =\boldsymbol{V}^{\prime} \boldsymbol{R}_{1} \boldsymbol{V}-\frac{1}{\tau_{\varepsilon}^{2}} \boldsymbol{V}^{\prime} \boldsymbol{R}_{1} \boldsymbol{V} \boldsymbol{R}_{2}^{-1} \boldsymbol{V}^{\prime} \boldsymbol{R}_{1} \boldsymbol{V} \\
\boldsymbol{L}_{1} & =\left(\boldsymbol{U}^{\prime} \boldsymbol{U}\right)^{-1} \boldsymbol{U}^{\prime} \boldsymbol{V}\left(\hat{\boldsymbol{\beta}}_{\text {pen }}-\boldsymbol{\beta}_{\text {pen }}\right), \boldsymbol{L}_{2}=\frac{1}{\tau_{\varepsilon}^{2}} \boldsymbol{R}_{2}^{-1} \boldsymbol{V}^{\prime} \boldsymbol{R}_{1} \boldsymbol{V} \hat{\boldsymbol{\beta}}_{p e n} \\
\mathrm{SSR} & =(\boldsymbol{\xi}-\hat{\boldsymbol{\xi}})^{\prime} \boldsymbol{X}^{\prime} \boldsymbol{X}(\boldsymbol{\xi}-\hat{\boldsymbol{\xi}})=\left(\boldsymbol{\xi}-\left(\boldsymbol{X}^{\prime} \boldsymbol{X}\right)^{-} \boldsymbol{X} \boldsymbol{y}\right)^{\prime} \boldsymbol{X}^{\prime} \boldsymbol{X}\left(\boldsymbol{\xi}-\left(\boldsymbol{X}^{\prime} \boldsymbol{X}\right)^{-} \boldsymbol{X} \boldsymbol{y}\right) \\
& =\left(\boldsymbol{\beta}_{\text {unpen }}-\hat{\boldsymbol{\beta}}_{\text {unpen }}-\boldsymbol{L}_{1}\right)^{\prime} \boldsymbol{U}^{\prime} \boldsymbol{U}\left(\boldsymbol{\beta}_{\text {unpen }}-\hat{\boldsymbol{\beta}}_{\text {unpen }}-\boldsymbol{L}_{1}\right)+\left(\boldsymbol{\beta}_{\text {pen }}-\hat{\boldsymbol{\beta}}_{\text {pen }}\right)^{\prime} \boldsymbol{V}^{\prime} \boldsymbol{R}_{1} \boldsymbol{V}\left(\boldsymbol{\beta}_{\text {pen }}-\hat{\boldsymbol{\beta}}_{\text {pen }}\right)
\end{aligned}
$$

and note that since $\boldsymbol{R}_{1}$ in (14) is non-negative definite this holds also for $\boldsymbol{V}^{\prime} \boldsymbol{R}_{1} \boldsymbol{V}$. Furthermore, $\boldsymbol{R}_{2}$ is positive definite since $\boldsymbol{T}$ is positive definite and hence $\boldsymbol{R}_{2}^{-1}$ exists. Finally, $\boldsymbol{R}_{3}$ is non-negative definite.

Under the conditional independence assumptions in Section 3.1 the posterior distribution $p\left(\boldsymbol{\xi}, \tau_{1}^{2}, \ldots, \tau_{J}^{2}, \tau_{\varepsilon}^{2} \mid \boldsymbol{y}\right)$ is proportional to

$$
\begin{aligned}
& \left(\tau_{\varepsilon}^{2}\right)^{-\frac{n}{2}} \exp \left(\frac{1}{2 \tau_{\varepsilon}^{2}}(\boldsymbol{y}-\boldsymbol{X} \boldsymbol{\xi})^{\prime}(\boldsymbol{y}-\boldsymbol{X} \boldsymbol{\xi})\right)\left(\tau_{\varepsilon}^{2}\right)^{-\left(a_{\varepsilon}+1\right)} \exp \left(-\frac{\theta_{\varepsilon}}{\tau_{\varepsilon}^{2}}\right) \\
& \cdot \prod_{j=1}^{J}\left[\left(\tau_{j}^{2}\right)^{-\frac{\kappa_{j}}{2}} \exp \left(\frac{1}{2 \tau_{j}^{2}} \boldsymbol{\beta}_{p e n, j}^{\prime} \boldsymbol{I}_{\kappa_{j}} \boldsymbol{\beta}_{p e n, j}\right)\left(\tau_{j}^{2}\right)^{-\frac{1}{2}} \exp \left(-\left(\tau_{j}^{2} / \theta_{j}\right)^{1 / 2}\right)\right]
\end{aligned}
$$

From Theorem 1 of Sun et al. (2001), integrating over $\boldsymbol{\xi}$ we obtain that the posterior is proper if and only if $\int_{0}^{\infty} G \mathrm{~d} \tau_{\varepsilon}^{2}<\infty$ holds with

$$
G \propto \frac{\left|\boldsymbol{U}^{\prime} \boldsymbol{U}\right|^{-1 / 2}}{\left(\tau_{\varepsilon}^{2}\right)^{(n-r) / 2+a_{\varepsilon}+1}} \exp \left(-\frac{\mathrm{SSE}+2 \theta_{\varepsilon}+\hat{\boldsymbol{\beta}}_{p e n}^{\prime} \boldsymbol{R}_{3} \hat{\boldsymbol{\beta}}_{p e n}}{2 \tau_{\varepsilon}^{2}}\right) G_{2}
$$

where

$$
G_{2} \propto \int_{0}^{\infty} \ldots \int_{0}^{\infty} G_{1} \mathrm{~d} \tau_{1}^{2} \ldots \mathrm{d} \tau_{J}^{2}
$$

and

$$
G_{1} \propto \frac{1}{\left|\boldsymbol{R}_{2}\right|^{1 / 2}|\boldsymbol{T}|^{1 / 2}} \prod_{j=1}^{J}\left(\tau_{j}^{2}\right)^{-\frac{1}{2}} \exp \left(-\left(\tau_{j}^{2} / \theta_{j}\right)^{1 / 2}\right)
$$


Case 1: $t=\kappa=\sum_{j=1}^{J} \kappa_{j}=\operatorname{dim}\left(\boldsymbol{\beta}_{\text {pen }}\right)$. Then, $\boldsymbol{V}^{\prime} \boldsymbol{R}_{1} \boldsymbol{V}$ is positive definite and has real positive eigenvalues $0<\lambda_{\min }<\ldots<\lambda_{\max }$. Using Lemma 2 of Sun et al. (2001) we obtain

$$
\begin{aligned}
\left|\boldsymbol{R}_{2}\right| & =\left|\frac{1}{\tau_{\varepsilon}^{2}} \boldsymbol{V}^{\prime} \boldsymbol{R}_{1} \boldsymbol{V}+\operatorname{diag}\left(\tau_{1}^{2} \boldsymbol{I}_{\kappa_{1}}, \ldots, \tau_{J}^{2} \boldsymbol{I}_{\kappa_{J}}\right)\right| \\
& \leq \prod_{j=1}^{J}\left[\left(\tau_{j}^{2}\right)^{-\kappa_{j}} \prod_{l=1}^{\kappa_{j}}\left(\tau_{j}^{2} \frac{\lambda_{\max }}{\tau_{\varepsilon}^{2}}+1\right)\right] \\
& =\prod_{j=1}^{J}\left[\left(\tau_{j}^{2}\right)^{-\kappa_{j}}\left(\tau_{j}^{2} \frac{\lambda_{\max }}{\tau_{\varepsilon}^{2}}+1\right)^{\kappa_{j}}\right] .
\end{aligned}
$$

With $|\boldsymbol{T}|^{-1}=\prod_{j=1}^{J}\left(\tau_{j}^{2}\right)^{-\kappa_{j}}$ it hence follows

$$
\begin{aligned}
\frac{1}{\left|\boldsymbol{R}_{2}\right|^{1 / 2}|\boldsymbol{T}|^{1 / 2}} & \geq \prod_{j=1}^{J}\left[\left(\tau_{j}^{2}\right)^{-\kappa_{j} / 2}\right] \prod_{j=1}^{J}\left[\left(\tau_{j}^{2}\right)^{\kappa_{j} / 2}\left(\tau_{j}^{2} \frac{\lambda_{\max }}{\tau_{\varepsilon}^{2}}+1\right)^{-\kappa_{j} / 2}\right] \\
& =\prod_{j=1}^{J}\left(\tau_{j}^{2} \frac{\lambda_{\max }}{\tau_{\varepsilon}^{2}}+1\right)^{-\kappa_{j} / 2}
\end{aligned}
$$

and

$$
G_{2} \geq \prod_{j=1}^{J} \int_{0}^{\infty}\left(\tau_{j}^{2}\right)^{-1 / 2} \exp \left(-\left(\tau_{j}^{2} / \theta_{j}\right)^{1 / 2}\right)\left(\tau_{j}^{2} \frac{\lambda_{\max }}{\tau_{\varepsilon}^{2}}+1\right)^{-\kappa_{j} / 2} \mathrm{~d} \tau_{j}^{2} .
$$

We then substitute $s=\frac{\tau_{j}^{2} \lambda_{\max }}{\tau_{\varepsilon}^{2}}$ and define $\theta_{j}^{*}=\frac{\theta_{j} \lambda \max }{\tau_{\varepsilon}^{2}}$ to arrive at

$$
G_{2} \geq\left(\frac{\tau_{\varepsilon}^{2}}{\lambda_{\max }}\right)^{J / 2} \prod_{j=1}^{J} \int_{0}^{\infty} s^{-1 / 2} \exp \left(-\left(s / \theta_{j}^{*}\right)^{1 / 2}\right)\left(\frac{1}{s+1}\right)^{\kappa_{j} / 2} \mathrm{~d} s .
$$

With Lemma 2,2 it follows that a lower bound for $G_{2}$ for all $\kappa_{j}$ and $\theta_{j}>0$ is given by

$$
G_{2} \geq\left(\frac{\tau_{\varepsilon}^{2}}{\lambda_{\max }}\right)^{J / 2} \prod_{j=1}^{J} \begin{cases}\frac{2 \exp \left(-\left(\theta_{j}^{*}\right)^{-1 / 2}\right)}{1-\kappa_{j}}\left((1 / 2)^{\left(\kappa_{j}-1\right) / 2}-1\right) & \kappa_{j} \neq 1 \\ \exp \left(-\left(\theta_{j}^{*}\right)^{-1 / 2}\right) \log (2) & \kappa_{j}=1 .\end{cases}
$$

Using the lower bound of Lemma 2 of Sun et al. (2001) and defining $\tilde{\theta}_{j}=\frac{\theta_{j} \lambda \min }{\tau_{\varepsilon}^{2}}$ gives the lower bound

$$
G_{2} \leq\left(\frac{\tau_{\varepsilon}^{2}}{\lambda_{\min }}\right)^{J / 2} \prod_{j=1}^{J} \int_{0}^{\infty} s^{-1 / 2}\left(\frac{1}{s+1}\right)^{\kappa_{j} / 2} \mathrm{~d} s
$$

which due to Lemma 2,2 is finite if Condition b.1 holds and in this case is bounded by

$$
G_{2} \leq\left(\frac{\tau_{\varepsilon}^{2}}{\lambda_{\min }}\right)^{J / 2} \prod_{j=1}^{J} \mathrm{~B}\left(\frac{1}{2}, \frac{\kappa_{j}-1}{2}\right)
$$

For some values $C_{1}, C_{2}$ not depending on $\tau_{\varepsilon}^{2}$, we thus obtain

$$
G \geq C_{1} \exp \left(-\frac{\mathrm{SSE}+2 \theta_{\varepsilon}+\hat{\boldsymbol{\beta}}_{p e n}^{\prime} \boldsymbol{R}_{3} \hat{\boldsymbol{\beta}}_{p e n}}{2 \tau_{\varepsilon}^{2}}\right)\left(\tau_{\varepsilon}^{2}\right)^{-\frac{n-r-J}{2}-a_{\varepsilon}-1} \exp \left(-\sum_{j=1}^{J}\left(\frac{\tau_{\varepsilon}^{2}}{\theta_{j} \lambda_{\max }}\right)^{\frac{1}{2}}\right) .
$$


Integrating over $\tau_{\varepsilon}^{2}$ gives

$$
\begin{aligned}
& \int_{0}^{\infty} G \mathrm{~d} \tau_{\varepsilon}^{2} \\
& \geq \int_{0}^{\infty} C_{1} \exp \left(-\frac{\mathrm{SSE}+2 \theta_{\varepsilon}+\hat{\boldsymbol{\beta}}_{p e n}^{\prime} \boldsymbol{R}_{3} \hat{\boldsymbol{\beta}}_{\text {pen }}}{2 \tau_{\varepsilon}^{2}}\right)\left(\tau_{\varepsilon}^{2}\right)^{-\frac{n-r-J}{2}-a_{\varepsilon}-1} \exp \left(-\sum_{j=1}^{J}\left(\frac{\tau_{\varepsilon}^{2}}{\theta_{j} \lambda_{\max }}\right)^{\frac{1}{2}}\right) \mathrm{d} \tau_{\varepsilon}^{2} \\
& \geq \int_{0}^{\infty} C_{1} \exp \left(-\frac{\mathrm{SSE}+2 \theta_{\varepsilon}+\hat{\boldsymbol{\beta}}_{\text {pen }}^{\prime} \boldsymbol{R}_{3} \hat{\boldsymbol{\beta}}_{\text {pen }}}{2 \tau_{\varepsilon}^{2}}\right)\left(\tau_{\varepsilon}^{2}\right)^{-\frac{n-r-J}{2}-a_{\varepsilon}-1} \exp \left(-\tau_{\varepsilon}^{2} \sum_{j=1}^{J}\left(\theta_{j} \lambda_{\max }\right)^{-\frac{1}{2}}\right) \mathrm{d} \tau_{\varepsilon}^{2}
\end{aligned}
$$

which is the integral over the density of a generalised inverse Gaussian distribution, i.e. $f(x) \propto x^{p-1} \exp (-(a x+b / x) / 2)$. Hence, the integral is finite if $a=$ $\sum_{j=1}^{J}\left(\theta_{j} \lambda_{\text {max }}\right)^{-1 / 2}>0, b=\mathrm{SSE}+2 \theta_{\varepsilon}+\hat{\boldsymbol{\beta}}_{p e n}^{\prime} \boldsymbol{R}_{3} \hat{\boldsymbol{\beta}}_{\text {pen }}>0$ and $p=(J-n-r) / 2-a_{\varepsilon} \in \mathbb{R}$ giving the necessary Condition (b.3) .

The upper bound for $G$ can finally be expressed as

$$
G \leq C_{2} \exp \left(-\frac{\mathrm{SSE}+2 \theta_{\varepsilon}}{2 \tau_{\varepsilon}^{2}}\right)\left(\tau_{\varepsilon}^{2}\right)^{-\frac{n-r-J}{2}-a_{\varepsilon}-1}
$$

which is the density of an inverse gamma distribution, i.e. $f(x) \propto x^{-a-1} \exp (-b / x)$, if $a=(n-r-J) / 2+a_{\varepsilon}>0$ and $b=\left(\mathrm{SSE}+2 \theta_{\varepsilon}\right) / 2>0$ and which gives the sufficient Conditions (b.2) and (b.3).

Case 2: $t<\kappa=\sum_{j=1}^{J} \kappa_{j}, J=1$. In particular this means $\kappa=\kappa_{1}$. Since $\operatorname{rk}\left(\boldsymbol{V}^{\prime} \boldsymbol{R}_{1} \boldsymbol{V}\right)=t$ holds, let $\lambda_{s p}$ and $\lambda_{\max }$ be the minimal positive and maximal positive eigenvalue out of the $t$ positive eigenvalues of $\boldsymbol{V}^{\prime} \boldsymbol{R}_{1} \boldsymbol{V}$. Using the same arguments as for Case 1, $G_{1}$ in (A.3) can thus be bounded by

$$
\frac{\tilde{C}_{1}}{\sqrt{\tau_{1}^{2}}} \exp \left(-\left(\frac{\tau_{1}^{2}}{\theta_{1}}\right)^{\frac{1}{2}}\right)\left(\frac{\tau_{1}^{2} \lambda_{\max }}{\tau_{\varepsilon}^{2}}+1\right)^{-\frac{t}{2}} \leq G_{1} \leq \frac{\tilde{C}_{2}}{\sqrt{\tau_{1}^{2}}} \exp \left(-\left(\frac{\tau_{1}^{2}}{\theta_{1}}\right)^{\frac{1}{2}}\right)\left(\frac{\tau_{1}^{2} \lambda_{s p}}{\tau_{\varepsilon}^{2}}+1\right)^{-\frac{t}{2}}
$$

for some values $\tilde{C}_{1}, \tilde{C}_{2}$ independent of $\tau_{\varepsilon}^{2}, \tau_{1}^{2}, \ldots, \tau_{J}^{2}$ which yields the same conditions as for Case 1.

Case 3: $t<\kappa=\sum_{j=1}^{J} \kappa_{j}, J>1$. Due to $\lambda_{\max }>0$, the necessary condition still holds. For sufficiency note that $\boldsymbol{V}^{\prime} \boldsymbol{R}_{1} \boldsymbol{V}$ has $\kappa-t$ zero eigenvalues. From Lemma 2 of Sun et al. (2001) it hence follows

$$
\begin{aligned}
\frac{1}{\left|\boldsymbol{R}_{2}\right|^{1 / 2}|\boldsymbol{T}|^{1 / 2}} & \leq \prod_{j=1}^{J}\left[\left(\tau_{j}^{2}\right)^{-\kappa_{j} / 2}\right] \prod_{j=1}^{J}\left[\left(\tau_{j}^{2}\right)^{\kappa_{j} / 2}\left(\tau_{j}^{2} \frac{\lambda_{s p}}{\tau_{\varepsilon}^{2}}+1\right)^{-\frac{t-1-\kappa+\kappa_{j}}{2}}\right] \\
& =\prod_{j=1}^{J}\left(\tau_{j}^{2} \frac{\lambda_{s p}}{\tau_{\varepsilon}^{2}}+1\right)^{-\frac{t-1-\kappa+\kappa_{j}}{2}} .
\end{aligned}
$$

From Lemma $2 \gamma=\frac{\kappa_{j}-\kappa+t}{2}>\frac{1}{2}$ is required which is equivalent to $\kappa_{j}>1+\kappa-t$. 


\section{A.4 Proof of Theorem 3}

For the proof we modify the proof of Theorem 1 of Klein et al. (2015) by using the results of Theorem [2. Define first of all for $k=1, \ldots, K$ the vectors $\tau_{k}^{2}=$ $\left(\tau_{1, k}^{2}, \ldots, \tau_{J_{k}^{*}, k}^{2}\right)^{\prime}$. Then, it is clear that

$p\left(\boldsymbol{\beta}_{\text {unpen }, 1}, \ldots, \boldsymbol{\beta}_{\text {unpen }, K}, \boldsymbol{b}_{1}, \ldots, \boldsymbol{b}_{K}, \boldsymbol{b}_{\varepsilon, 1}, \ldots, \boldsymbol{b}_{\varepsilon, K}, \boldsymbol{\tau}_{1}^{2}, \ldots, \boldsymbol{\tau}_{K}^{2}, \tau_{\varepsilon, 1}^{2}, \ldots, \tau_{\varepsilon, K}^{2} \mid \boldsymbol{y}\right)$ proper

$\Leftrightarrow$

$p\left(\boldsymbol{\beta}_{\text {unpen }, 1}, \ldots, \boldsymbol{\beta}_{\text {unpen }, K}, \boldsymbol{b}_{1}, \ldots, \boldsymbol{b}_{K}, \tilde{\boldsymbol{\eta}}_{1, s}, \ldots, \tilde{\boldsymbol{\eta}}_{K, s}, \boldsymbol{\tau}_{1}^{2}, \ldots, \boldsymbol{\tau}_{K}^{2}, \tau_{\varepsilon, 1}^{2}, \ldots, \tau_{\varepsilon, K}^{2} \mid \boldsymbol{y}\right)$ proper.

From the properties of the mixed model representation and the conditional independence assumptions, the posterior is proportional to

$$
\begin{aligned}
& \prod_{i=1}^{n} p\left(y_{i} \mid \eta_{i 1}, \ldots, \eta_{i K}\right) \prod_{k=1}^{K}\left[p\left(\tilde{\boldsymbol{\eta}}_{k, s} \mid \boldsymbol{\beta}_{\text {unpen }, k}, \boldsymbol{b}_{k}, \tau_{\varepsilon, k}^{2}\right) p\left(\tau_{\varepsilon, k}^{2}\right) \prod_{j=1}^{J_{k}^{*}}\left[p\left(\boldsymbol{b}_{j, k} \mid \tau_{j, k}^{2}\right) p\left(\tau_{j, k}^{2}\right)\right]\right] \\
\propto & \prod_{i=1}^{n} p\left(y_{i} \mid \eta_{i 1}, \ldots, \eta_{i K}\right) \tilde{G}
\end{aligned}
$$

with

$$
\begin{aligned}
\tilde{G}= & \prod_{k=1}^{K}\left[\left(\frac{1}{\tau_{\varepsilon, k}^{2}}\right)^{\frac{\tilde{k}_{\varepsilon}}{2}} \exp \left(-\frac{1}{\tau_{\varepsilon, k}^{2}}\left(\tilde{\boldsymbol{\eta}}_{k, s}-\tilde{\boldsymbol{U}}_{k, s} \boldsymbol{\beta}_{\text {unpen }, k}-\tilde{\boldsymbol{V}}_{k, s} \boldsymbol{b}_{k}\right)^{\prime}\left(\tilde{\boldsymbol{\eta}}_{k, s}-\tilde{\boldsymbol{U}}_{k, s} \boldsymbol{\beta}_{\text {unpen }, k}-\tilde{\boldsymbol{V}}_{k, s} \boldsymbol{b}_{k}\right)\right)\right] \\
& \cdot \prod_{k=1}^{K}\left[\left(\frac{1}{\left|\boldsymbol{Q}_{k}\right|}\right)^{1 / 2} \exp \left(-\frac{\boldsymbol{b}_{k}^{\prime} \boldsymbol{Q}_{k} \boldsymbol{b}_{k}}{2}\right) p\left(\boldsymbol{\tau}_{k}^{2}\right) p\left(\tau_{\varepsilon, k}^{2}\right)\right]
\end{aligned}
$$

and $\boldsymbol{Q}_{k}=\operatorname{Cov}\left(\boldsymbol{b}_{k}\right)$. Define $M^{*}=M^{n-n^{*}}$ and use Condition (c.2) to get

$$
\begin{aligned}
& p\left(\boldsymbol{\beta}_{\text {unpen }, 1}, \ldots, \boldsymbol{\beta}_{\text {unpen }, K}, \boldsymbol{b}_{1}, \ldots, \boldsymbol{b}_{K}, \tilde{\boldsymbol{\eta}}_{1, s}, \ldots, \tilde{\boldsymbol{\eta}}_{K, s}, \boldsymbol{\tau}_{1}^{2}, \ldots, \boldsymbol{\tau}_{K}^{2}, \tau_{\varepsilon, 1}^{2}, \ldots, \tau_{\varepsilon, K}^{2} \mid \boldsymbol{y}\right) \\
& \leq M^{*} \prod_{i=1}^{n^{*}} p\left(y_{i} \mid \eta_{i 1}, \ldots, \eta_{i K}\right) \tilde{G} .
\end{aligned}
$$

where further normalising constants are omitted here for convenience. Integrating over $\boldsymbol{\beta}_{\text {unpen }, 1}, \ldots, \boldsymbol{\beta}_{\text {unpen }, K}, \boldsymbol{b}_{1}, \ldots, \boldsymbol{b}_{K}, \boldsymbol{\tau}_{1}^{2}, \ldots, \boldsymbol{\tau}_{K}^{2}$ implies

$$
\begin{aligned}
& p\left(\tilde{\boldsymbol{\eta}}_{1, s}, \ldots, \tilde{\boldsymbol{\eta}}_{K, s}, \boldsymbol{\tau}_{1}^{2}, \ldots, \boldsymbol{\tau}_{K}^{2}, \tau_{\varepsilon, 1}^{2}, \ldots, \tau_{\varepsilon, K}^{2} \mid \boldsymbol{y}\right) \\
& \leq M^{*} \prod_{i=1}^{n} p\left(y_{i} \mid \eta_{i}^{\vartheta_{1}}, \ldots, \eta_{i}^{\vartheta_{m}}\right) \int \ldots \int \tilde{G} \mathrm{~d} \boldsymbol{\beta}_{\text {unpen }, 1} \ldots \mathrm{d} \boldsymbol{\beta}_{\text {unpen }, K} \mathrm{~d} \boldsymbol{b}_{1} \ldots \mathrm{d} \boldsymbol{b}_{K} \mathrm{~d} \boldsymbol{\tau}_{1}^{2} \ldots \mathrm{d} \boldsymbol{\tau}_{K}^{2} .
\end{aligned}
$$

The integral over $\tilde{G}$ corresponds to $G_{2}$ in (A.1) which can be bounded similarly to (A.6) under Condition (c.6) leading to the inequality

$$
\begin{aligned}
& p\left(\tilde{\boldsymbol{\eta}}_{1, s}, \ldots, \tilde{\boldsymbol{\eta}}_{K, s}, \boldsymbol{\tau}_{1}^{2}, \ldots, \boldsymbol{\tau}_{K}^{2}, \tau_{\varepsilon, 1}^{2}, \ldots, \tau_{\varepsilon, K}^{2} \mid \boldsymbol{y}\right) \\
& \leq M^{*} \prod_{i=1}^{n} p\left(y_{i} \mid \eta_{i}^{\vartheta_{1}}, \ldots, \eta_{i}^{\vartheta_{m}}\right) \int \ldots \int \tilde{g}\left(\tau_{\varepsilon, 1}^{2}, \ldots, \tau_{\varepsilon, K}^{2}\right) \mathrm{d} \tau_{\varepsilon, 1}^{2}, \ldots, \mathrm{d} \tau_{\varepsilon, K}^{2}
\end{aligned}
$$


with

$$
\begin{aligned}
\tilde{g}\left(\tau_{\varepsilon, 1}^{2}, \ldots, \tau_{\varepsilon, K}^{2}\right)= & \tilde{C}_{3} \prod_{k=1}^{K}\left(\tau_{\varepsilon, k}^{2}\right)^{-\frac{\tilde{k}_{\varepsilon}-r_{k}-J_{k}^{*}+1}{2}} \exp \left(-\frac{\mathrm{SSE}_{k, s}}{2 \tau_{\varepsilon, k}^{2}}\right) \\
& \cdot \prod_{k=1}^{K} \exp \left(-\tau_{\varepsilon, k}^{2}\left[\theta_{\varepsilon}^{-1}+\sum_{j=1}^{J_{k}^{*}} \tilde{\theta}_{j}^{-0.5}\right]\right)
\end{aligned}
$$

and $\mathrm{SSE}_{k, s}$ defined in (19). Condition (c.7) is hence sufficient for $\tilde{g}$ being the density of a generalised inverse Gaussian distribution implying

$$
\int \ldots \int \tilde{g}\left(\tau_{\varepsilon, 1}^{2}, \ldots, \tau_{\varepsilon, K}^{2}\right) \mathrm{d} \tau_{\varepsilon, 1}^{2} \ldots \mathrm{d} \tau_{\varepsilon, K}^{2}<\infty
$$

and thus that for some constant $\tilde{C}_{4}$

$$
p\left(\tilde{\boldsymbol{\eta}}_{1, s}, \ldots, \tilde{\boldsymbol{\eta}}_{K, s} \mid \boldsymbol{y}\right) \leq \tilde{C}_{4} \prod_{i=1}^{n^{*}} p\left(y_{i} \mid \eta_{i 1}, \ldots, \eta_{i K}\right)
$$

holds. Finally, it remains to show

$$
\int \ldots \int p\left(\tilde{\boldsymbol{\eta}}_{1, s}, \ldots, \tilde{\boldsymbol{\eta}}_{K, s} \mid \boldsymbol{y}\right) \mathrm{d} \tilde{\boldsymbol{\eta}}_{1, s} \ldots \mathrm{d} \tilde{\boldsymbol{\eta}}_{K, s}<\infty .
$$

This follows directly from Condition (c.1) applying the change of variable theorem to $\tilde{\boldsymbol{\eta}}_{k, s}=\boldsymbol{M}_{k} \boldsymbol{\eta}_{k, s}$ :

$$
\begin{aligned}
& \int \ldots \int p\left(\tilde{\boldsymbol{\eta}}_{1, s}, \ldots, \tilde{\boldsymbol{\eta}}_{K, s} \mid \boldsymbol{y}\right)\left|\boldsymbol{M}_{1}^{-1}\right| \mathrm{d} \boldsymbol{\eta}_{1, s} \ldots\left|\boldsymbol{M}_{K}^{-1}\right| \mathrm{d} \boldsymbol{\eta}_{K, s} \\
& \leq \tilde{K} \int \ldots \int \prod_{i=1}^{n^{*}} p\left(y_{i} \mid \eta_{i 1}, \ldots, \eta_{i K}\right) \mathrm{d} \eta_{i 1} \ldots \mathrm{d} \eta_{i K}<\infty
\end{aligned}
$$

for some constant $\tilde{K}<\infty$.

\section{B Scale Parameters for Bayesian P-Splines}

While computation of the optimal scale parameter $\theta$ for given design matrix $\boldsymbol{Z}$ and (possibly partially improper) precision matrix $\boldsymbol{K}$ with respect to the integral (10) is provided within the R-package sdPrior, our aim in this section is to propose default values for cubic Bayesian P-splines with second order random walk prior which can be used by applied researchers in practice. Since the structure of $\boldsymbol{K}$ will only depend on the number of basis functions in this case, there are mainly the following parameters that may influence the value of $\theta$ : The design matrix $\boldsymbol{Z}$ and the Bonferroni inequality. In addition, we assume throughout this section that $c=3$ from (8) is a reasonable choice. The latter is motivated from the fact that with the most common link functions such as the log link, the probit link or the logistic link, there is no more variability in the desired parameter if the predictor exceeds the range from -3 to 3 . 
The rest of this section is structured as follows: Section B.1 investigates the loss of efficiency due to the Bonferroni inequality whereas Sections B.2 and B.3 empirically check the impact of the values for $x$.

If not stated otherwise, we fix parameters as follows:

- Let in the following $P$ denote the number of realisations of $x$ such that $\mathcal{X}_{P}=$ $\left\{x_{1}, \ldots, x_{P}\right\}$ is the set of covariates the function $f$ is evaluated at.

- The probability statements under consideration are $\alpha \in\{0.01,0.05,0.1,0.2,0.5$, $0.7\}$.

- Let $r=1, \ldots, R=1000$ be the number of simulation replications.

\section{B.1 Bonferroni Inequality}

In this setting, $\mathcal{X}_{P}$ is a deterministic equidistant grid within the interval $[-3,3]$ of sizes $P \in\{1,2,5,10,15,30,40,50,100\}$. First we note that the marginal coverage probability can be rewritten as

$$
\begin{aligned}
\mathbb{P}\left(-3 \leq \boldsymbol{z}_{p}^{\prime} \boldsymbol{\beta} \leq 3\right) & =\mathbb{E}_{\boldsymbol{z}_{p}^{\prime} \boldsymbol{\beta}}\left(\mathbb{1}_{(-3,3)}\left(\boldsymbol{z}_{p}^{\prime} \boldsymbol{\beta}\right)\right) \\
& =\mathbb{E}_{\tau^{2}}\left(\mathbb{E}_{\boldsymbol{z}_{p}^{\prime} \boldsymbol{\beta} \mid \tau^{2}}\left(\mathbb{1}_{(-3,3)}\right)\left(\boldsymbol{z}_{p}^{\prime} \boldsymbol{\beta}\right)\right) .
\end{aligned}
$$

The Monte Carlo results in Figure B1 have the been obtained as follows: Given $\boldsymbol{Z}$, we first compute $\theta$. Then, we draw 1000 realisations of $\tau^{2} \sim \operatorname{Weibull}(\theta)$ and for each $\tau^{2}$ another 1000 realisations of $\boldsymbol{\beta} \mid \tau^{2}$. Empirical means of (B.1) should then approximately be equal to $1-\alpha$ and $\geq 1-\alpha$ when this probability is desired for all $x \in \mathcal{X}_{P}$ simultaneously. The means are indicated by the black crosses in Figure B1, We conclude the following:

(1) As expected, the desired level is maintained pointwise for all $P$ while for the simultaneous statement, independently from $\alpha$, larger values of $P$ induce more conservative statements.

(2) As already mentioned in the paper, in general the usage of the Bonferroni inequality only makes the statement in (9) more conservative which is not of very high relevance for our purposes. Furthermore, in simulations of the next Section $\mathrm{G}$, the results for $\theta$ (and the resulting functional estimates for $f$ ) proved to be very robust across different values for $\alpha$. Consequently, we expect that the estimation results for the functional effects $f$ will change only marginally in practice.

(3) Figures B2 to B5 show the equivalent results of the Monte Carlo experiment (B.1) but with the alternative priors introduced in Section 2.3. In general, these plots give some empirical evidence of our optimisation routines to be implemented correctly. One specific issue arises however with the half-Cauchy prior and the coverages of Figure B4 for $\alpha$ small and $P$ large. The reason are numerical instabilities for such constellations of $\alpha$ and $P$ that forced us to set $\theta$ to a minimal value that approximates the desired marginal probabilities best. We expect this problem to be arising from the heavy tails of the half-Cauchy distribution. Note on the other hand, that later in our simulations and empirical evaluations we used $P=1$ in which case the desired coverage is maintained and not underestimated. 


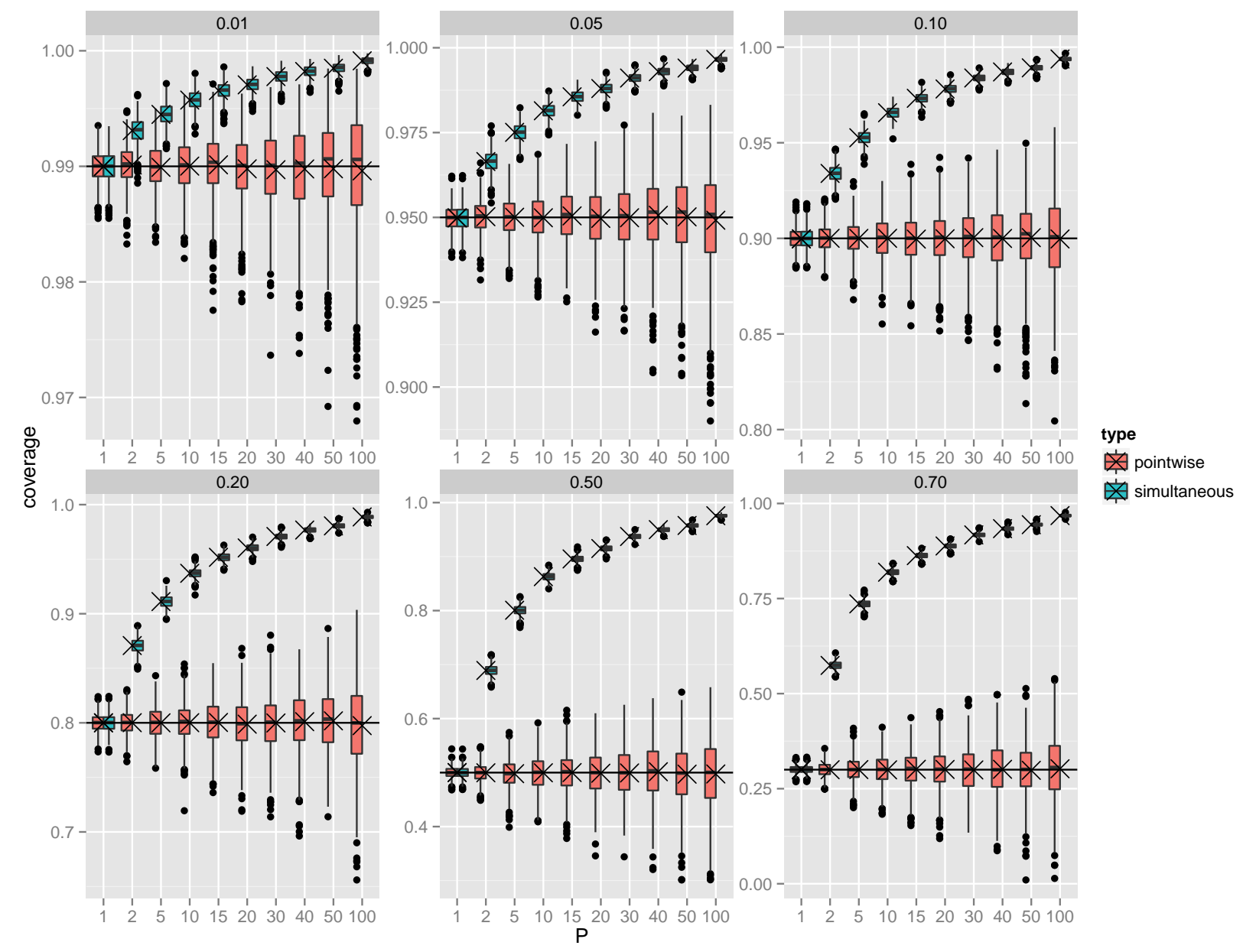

Figure B1: Scale-dependent prior for $\tau^{2}$. Default scale parameters for Bayesian P-splines. Impact of the Bonferroni inequality. Shown are pointwise (red) and simultaneous (blue) coverage probability statements, $\mathbb{P}\left(\left|f\left(\boldsymbol{x}_{p}\right)\right| \leq c, \boldsymbol{x} \in \mathcal{X}_{P}\right)$ and $1-\sum_{p=1}^{P} \mathbb{P}\left(\left|f\left(\boldsymbol{x}_{p}\right)\right| \geq c\right)$, respectively. Results are obtained from averages over 1000 draws for $\tau^{2}$ and 1000 draws for $\boldsymbol{\beta} \mid \tau^{2}$ each.

\section{B.2 Optimal Scale Parameter Based on Observed Covariate Values}

The distribution of $x$ varies between a uniform, normal and gamma distribution and the distribution parameters are chosen such that they have equal quantile ranges $d x \in$ $\{20,10,6,4,2,1\}$. The level $\alpha$ is fixed to 0.01 . Figure B6 shows the resulting optimal scale parameters $\theta$ in all situations. Results are averages over the $R$ replications. Important results are:

(1) As expected, the larger $P \in\{1,2,3,4,5,10,15,20\}$ the smaller $\theta$.

(2) For $P \geq 2$ all absolute values lie in the interval [0.002,0.005] such that the differences are relatively small in absolute terms. For $P=1$ we obtain values around 0.008 .

(3) The interval range $d x$ does not seem to influence $\theta$ even though some variation within the interval range is visible. 


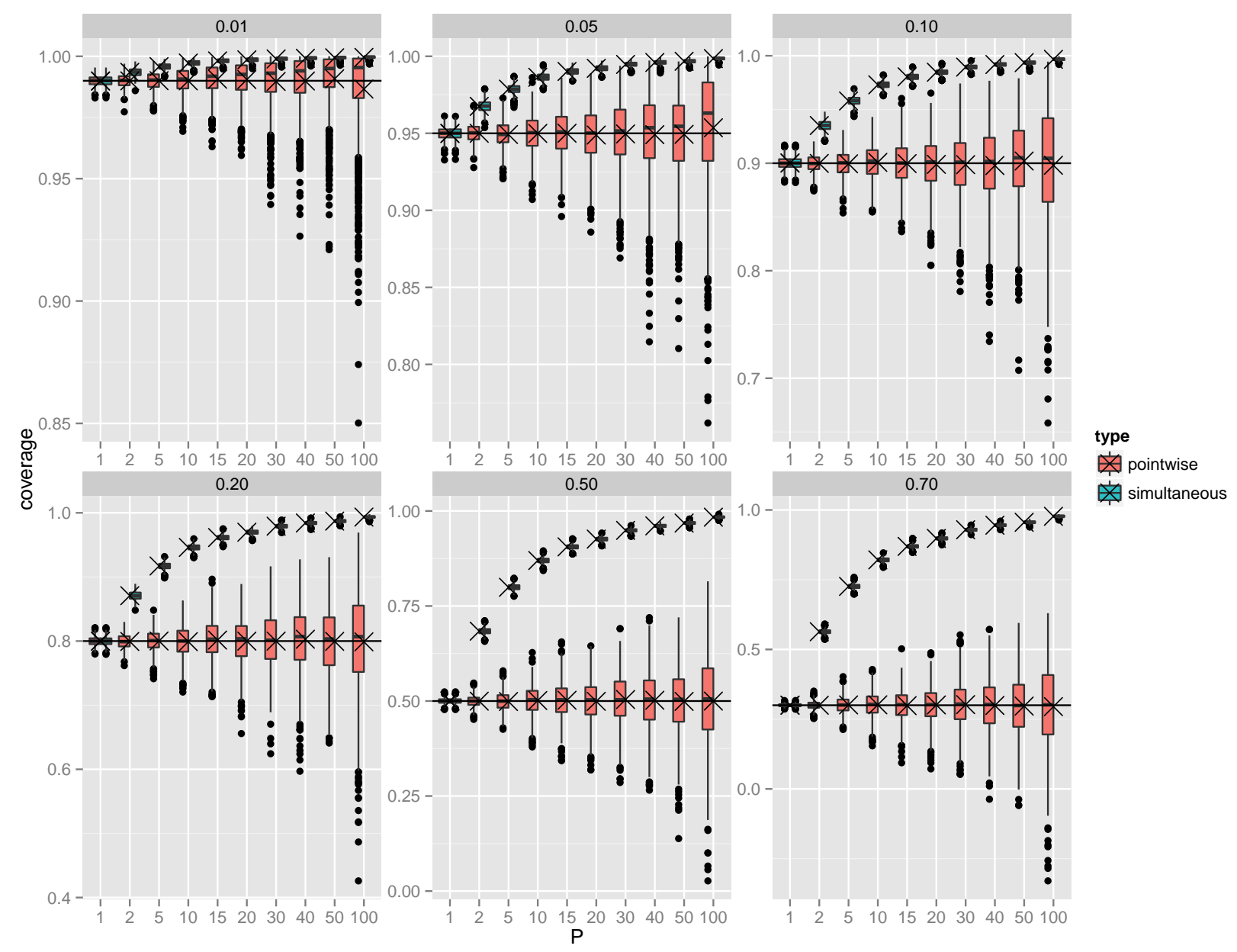

Figure B2: Inverse gamma prior for $\tau^{2}$. Default scale parameters for Bayesian P-splines. Impact of the Bonferroni inequality. Shown are pointwise (red) and simultaneous (blue) coverage probability statements, $\mathbb{P}\left(\left|f\left(\boldsymbol{x}_{p}\right)\right| \leq c, \boldsymbol{x} \in \mathcal{X}_{P}\right)$ and $1-\sum_{p=1}^{P} \mathbb{P}\left(\left|f\left(\boldsymbol{x}_{p}\right)\right| \geq c\right)$, respectively. Results are obtained from averages over 1000 draws for $\tau^{2}$ and 1000 draws for $\boldsymbol{\beta} \mid \tau^{2}$ each.

\section{B.3 Optimal Scale Parameter Based on Knots of the Design Matrix}

Let here $x_{1} \leq \ldots \leq x_{P}$ be the sequence of $P=22$ knots corresponding to the design matrices $\boldsymbol{Z}$ of cubic B-splines and seconder order random walk obtained for univariate, normal or gamma distributed covariates $x$ in samples of size $n=50,10,1000, d x$ as above and $\alpha=0.01$ as before. Figure B7 shows the resulting values for $\theta$. In line with the results from the previous section, for all three distributions, ranges $d x$ and sample sizes $n$, the optimal $\theta$ is approximately the same $(\theta \approx 0.003)$.

Based on the results so far, we decided for a pointwise selection of $\theta(P=1)$ in our simulations and applications with Bayesian P-splines (depending on the chosen values of $\alpha$ and $c$ ). Exemplary values for $c=3$ are listed in Table B1, The resulting marginal densities of $\boldsymbol{z}_{p}^{\prime} \boldsymbol{\beta}$ with $c=3$ and $\alpha=0.01$ are shown in Figure B8 and its computation has been integrated into the R-package sdPrior as well. 


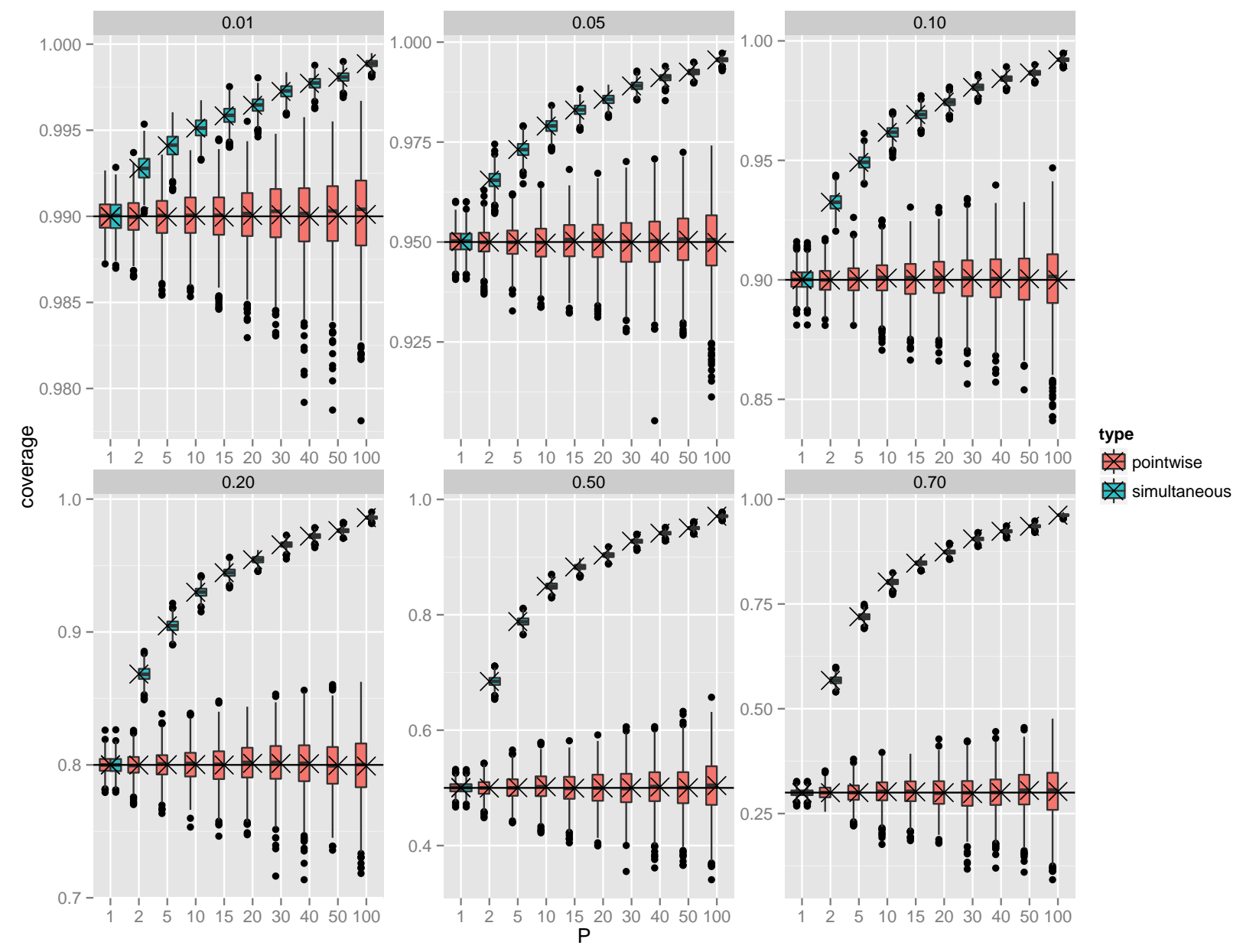

Figure B3: Half-normal prior for $\tau$. Default scale parameters for Bayesian P-splines. Impact of the Bonferroni inequality. Shown are pointwise (red) and simultaneous (blue) coverage probability statements, $\mathbb{P}\left(\left|f\left(\boldsymbol{x}_{p}\right)\right| \leq c, \boldsymbol{x} \in \mathcal{X}_{P}\right)$ and $1-\sum_{p=1}^{P} \mathbb{P}\left(\left|f\left(\boldsymbol{x}_{p}\right)\right| \geq c\right)$, respectively. Results are obtained from averages over 1000 draws for $\tau^{2}$ and 1000 draws for $\boldsymbol{\beta} \mid \tau^{2}$ each.

\begin{tabular}{l|cccccc}
\hline \hline$\alpha$ & 0.01 & 0.05 & 0.10 & 0.20 & 0.50 & 0.70 \\
\hline$\theta$ for $\mathrm{SD}(\alpha)$ & 0.0088 & 0.0307 & 0.0660 & 0.1826 & 1.9251 & 11.6246 \\
$\theta$ for $\mathrm{IG}(\alpha)$ & 0.0028 & 0.0149 & 0.0323 & 0.0776 & 0.4140 & 1.3952 \\
$\theta$ for $\mathrm{HN}(\alpha)$ & 0.1458 & 0.2407 & 0.3293 & 0.5078 & 1.4387 & 3.3006 \\
$\theta$ for $\mathrm{HC}(\alpha)$ & 0.0103 & 0.0520 & 0.1061 & 0.2277 & 0.8953 & 2.2565 \\
$\theta$ for $\mathrm{U}(\alpha)$ & 0.2724 & 0.4075 & 0.5297 & 0.7718 & 2.0089 & 4.4540 \\
\hline \hline
\end{tabular}

Table B1: Bayesian P-splines. Exemplary values for $\theta$ with $c=3$ and $P=1$. 


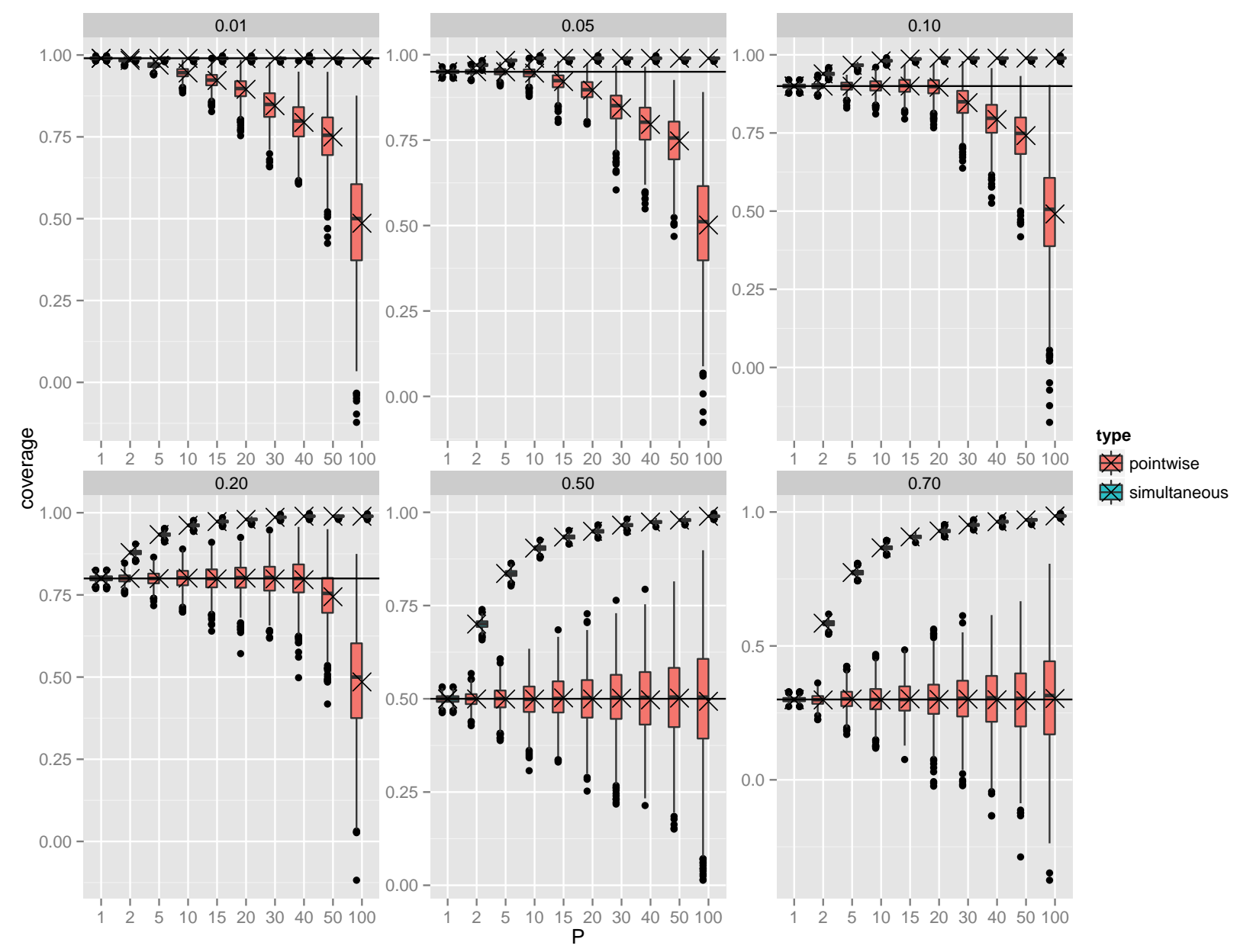

Figure B4: Half-Cauchy prior for $\tau$. Default scale parameters for Bayesian P-splines. Impact of the Bonferroni inequality. Shown are pointwise (red) and simultaneous (blue) coverage probability statements, $\mathbb{P}\left(\left|f\left(\boldsymbol{x}_{p}\right)\right| \leq c, \boldsymbol{x} \in \mathcal{X}_{P}\right)$ and $1-\sum_{p=1}^{P} \mathbb{P}\left(\left|f\left(\boldsymbol{x}_{p}\right)\right| \geq c\right)$, respectively. Results are obtained from averages over 1000 draws for $\tau^{2}$ and 1000 draws for $\boldsymbol{\beta} \mid \tau^{2}$ each. 


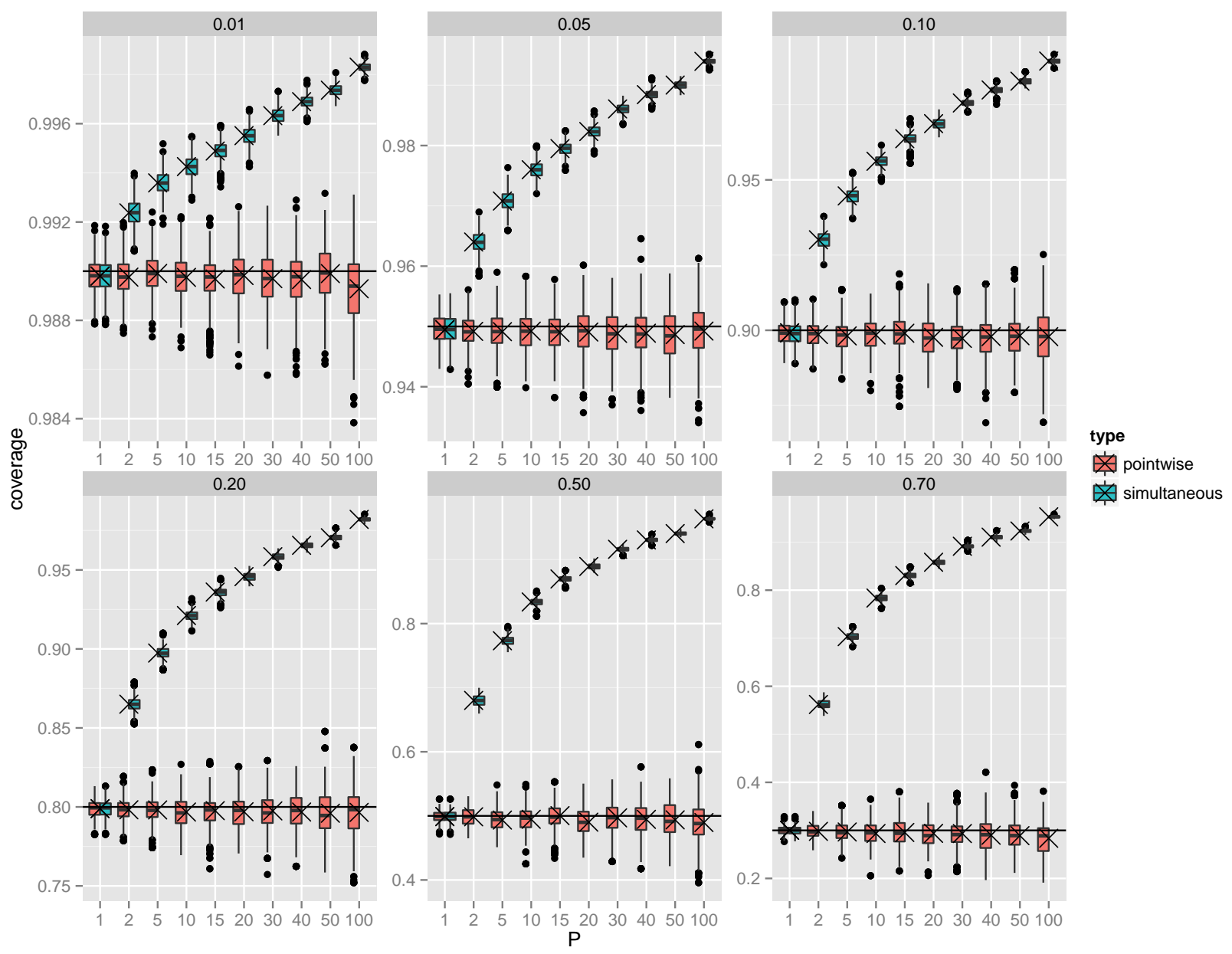

Figure B5: Approximate uniform prior for $\tau$. Default scale parameters for Bayesian P-splines. Impact of the Bonferroni inequality. Shown are pointwise (red) and simultaneous (blue) coverage probability statements, $\mathbb{P}\left(\left|f\left(\boldsymbol{x}_{p}\right)\right| \leq c, \boldsymbol{x} \in \mathcal{X}_{P}\right)$ and $1-\sum_{p=1}^{P} \mathbb{P}\left(\left|f\left(\boldsymbol{x}_{p}\right)\right| \geq c\right)$, respectively. Results are obtained from averages over 1000 draws for $\tau^{2}$ and 1000 draws for $\boldsymbol{\beta} \mid \tau^{2}$ each. 


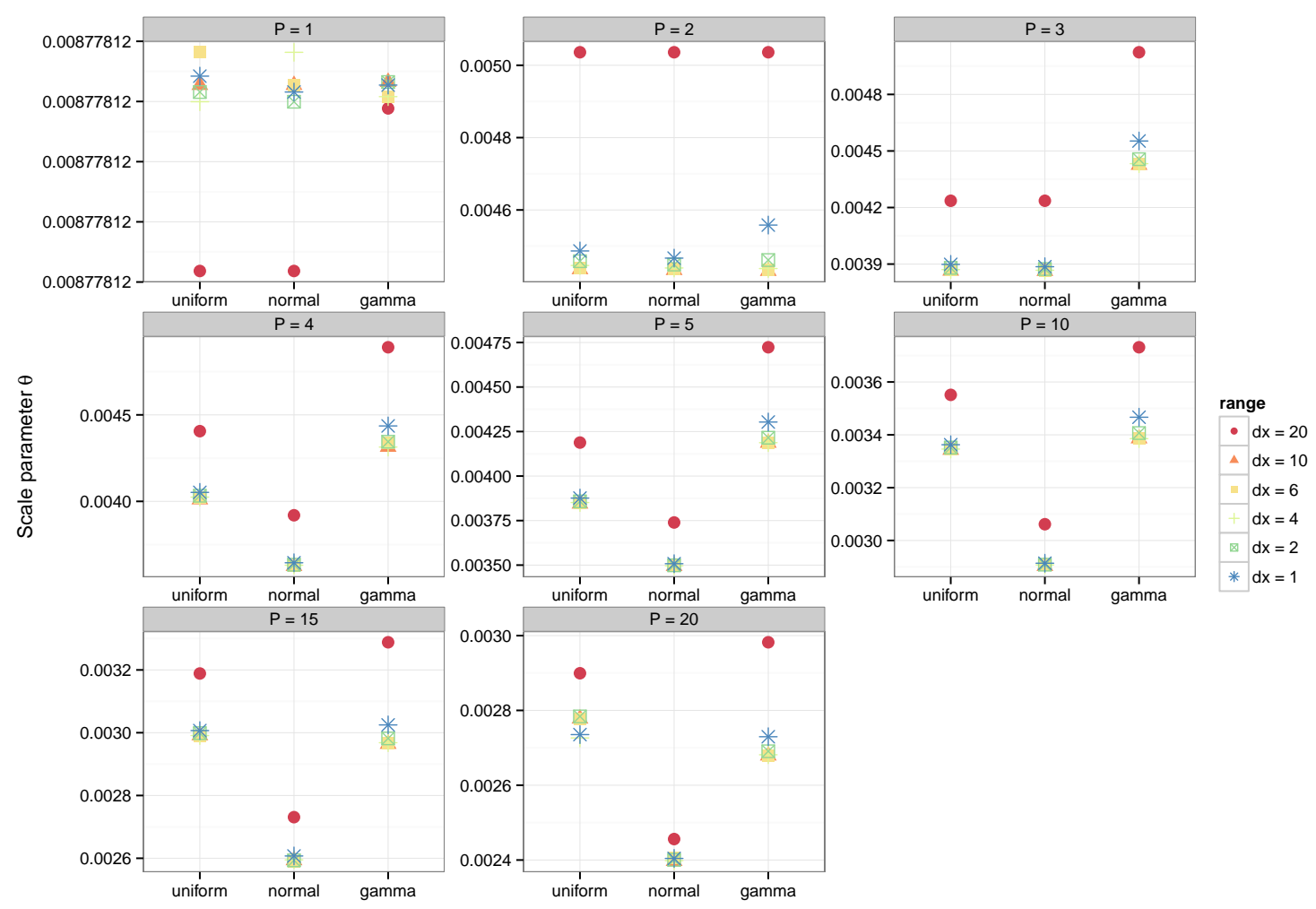

Figure B6: Default scale parameters for Bayesian P-splines. Shown are results from the get_theta function evaluated at covariates generated from various settings.

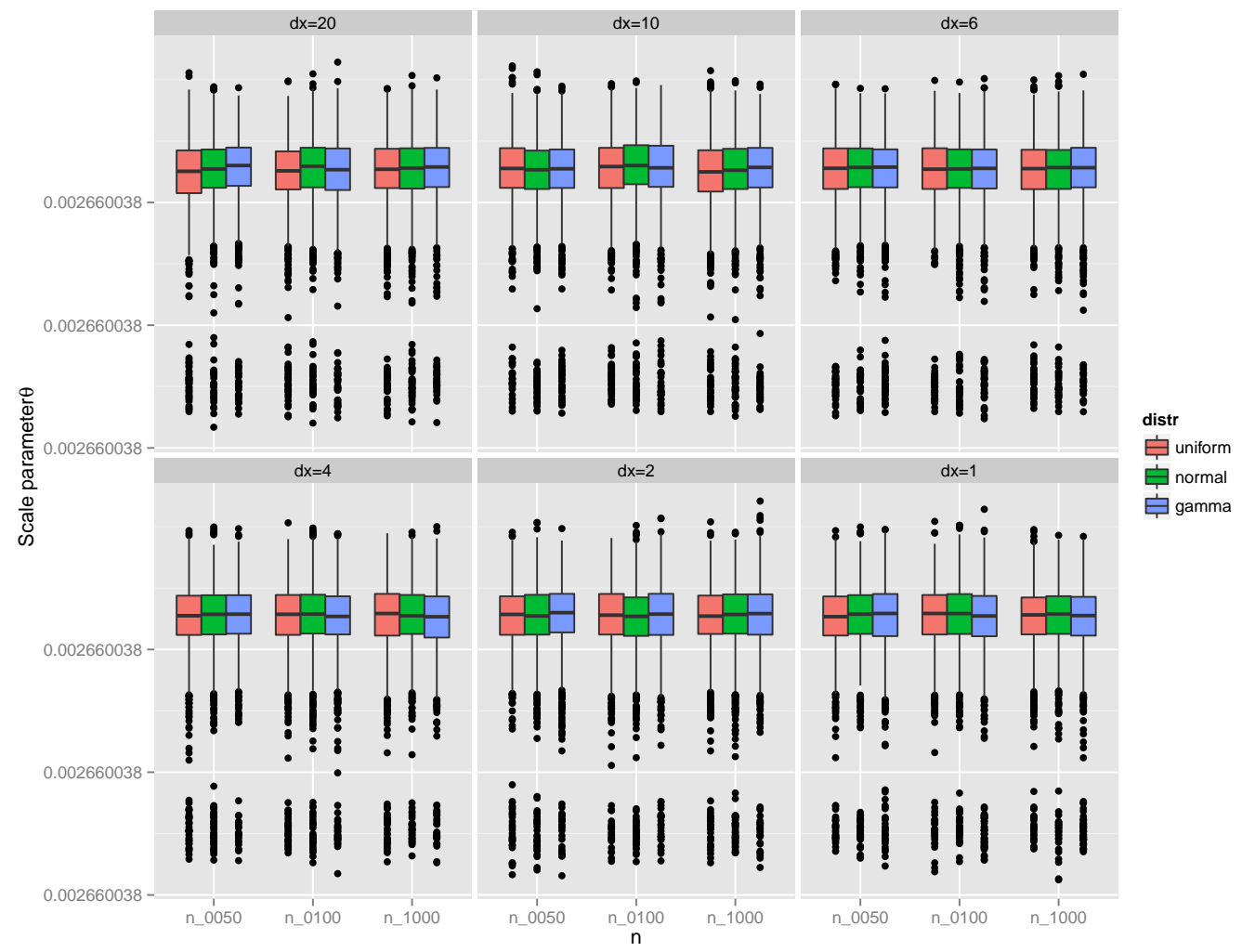

Figure B7: Default scale parameters for Bayesian P-splines. Shown are results from the get_theta function evaluated at the knots of the design matrix for given covariates. 

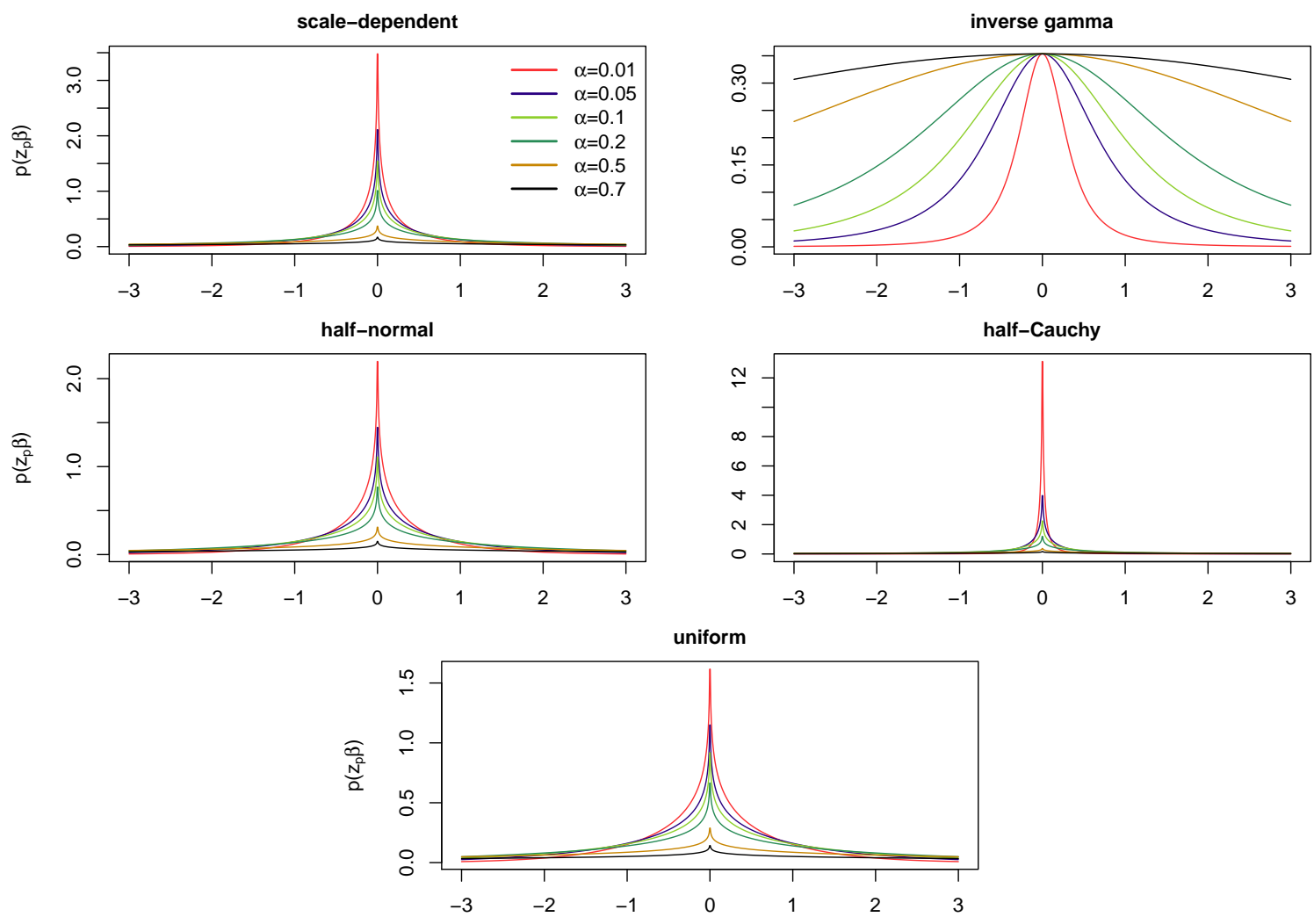

Figure B8: Illustration of marginal densities $p\left(\boldsymbol{z}_{p}^{\prime} \boldsymbol{\beta}\right)$ for different hyperpriors for $\tau^{2}$. The scale parameters $\theta$ of the priors is the value resulting from a pointwise optimisation criterion with $\alpha \in$ $\{0.01,0.05,0.1,0.2,0.5,0.7\}, c=3, P=1$ for a spline component with B-spline basis of degree 3,20 inner knots and second order difference matrix. 


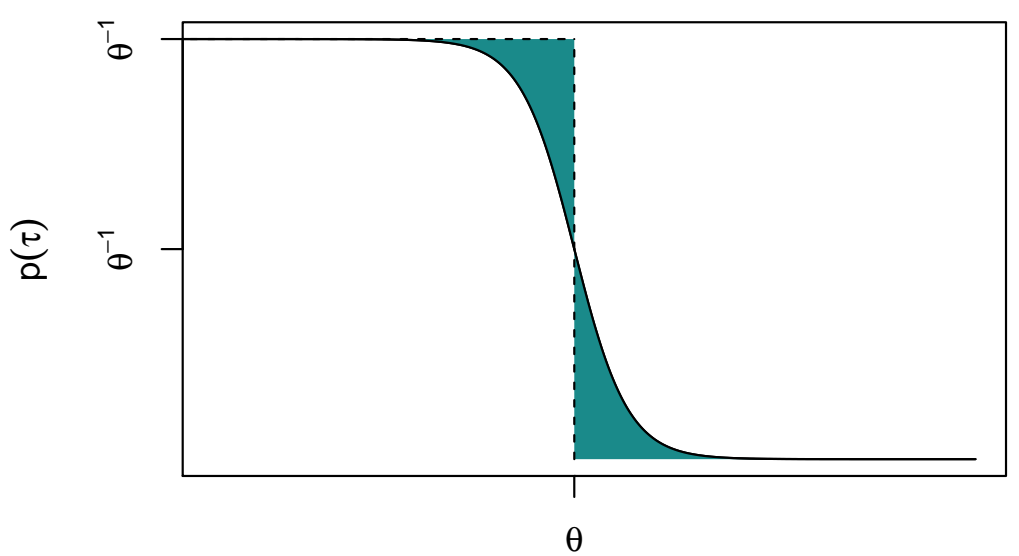

Figure C9: Illustration of the construction of an approximate uniform prior on $(0, \theta)$.

\section{Details on Approximate Uniform Prior for $\tau$}

\section{C.1 Densities for $\tau$ and $\tau^{2}$}

The general idea to obtain a suitable approximation of the uniform prior for $\tau$ is to approximate it with a sigmoid function tending to 1 for $\tau \rightarrow 0$ and to 0 for $\tau \rightarrow \theta$. An illustration is given in Figure C9. The sigmoid function $S(\tau)$ is defined as

$$
S(\tau)=\theta^{-1}\left(1-\frac{\exp (\tau \tilde{c} / \theta-\tilde{c})}{1+\exp (\tau \tilde{c} / \theta-\tilde{c})}\right)
$$

where $\tilde{c}$ should be chosen such that the green area highlighting the deviation between the uniform density and the approximation is small on the one hand and sufficiently large to be numerically tractable for our purposes on the other hand. To obtain a density for $\tau$, we compute the normalising constant as

$$
\begin{aligned}
1 & \stackrel{!}{=} \int_{0}^{\infty} \theta^{-1}\left(1-\frac{\exp (\tau \tilde{c} / \theta-\tilde{c})}{1+\exp (\tau \tilde{c} / \theta-\tilde{c})}\right) \mathrm{d} \tau \\
& =\int_{-\tilde{c}}^{\infty} \tilde{c}^{-1}\left(1-\frac{\exp (z)}{1+\exp (z)}\right) \mathrm{d} z \\
& =\tilde{c}^{-1}[\log (\exp (z))-\log (1+\exp (z))]_{-\tilde{c}}^{\infty} \\
& =\tilde{c}^{-1}\left[\log \left(\frac{\exp (z)}{1+\exp (z)}\right)\right]_{-\tilde{c}}^{\infty} \\
& =\tilde{c}^{-1}\left[\lim \log \left(\frac{\exp (z)}{1+\exp (z)}\right)-\log \left(\frac{\exp (-\tilde{c})}{1+\exp (-\tilde{c})}\right)\right] \\
& =\tilde{c}^{-1}[\underbrace{\log (1)}_{0}+\tilde{c}+\log (1+\exp (-\tilde{c}))] \\
& =1+\tilde{c}^{-1} \log (1+\exp (-\tilde{c}))
\end{aligned}
$$


where the substitution $z=\tau \tilde{c} / \theta-\tilde{c}$ has been used. Consequently, we define the approximate uniform prior as

$$
p_{\text {approx }}(\tau)=\frac{\theta^{-1}}{1+\log (1+\exp (-\tilde{c})) / \tilde{c}}\left(1-\frac{\exp (\tau \tilde{c} / \theta-\tilde{c})}{1+\exp (\tau \tilde{c} / \theta-\tilde{c})}\right) .
$$

Applying the change of variable theorem finally gives the prior for $\tau^{2}$ :

$$
p\left(\tau^{2}\right)=\frac{(2 \theta)^{-1}\left(\tau^{2}\right)^{-1 / 2}}{1+\log (1+\exp (-\tilde{c})) / \tilde{c}}\left(1-\frac{\exp \left(\left(\tau^{2}\right)^{1 / 2} \tilde{c} / \theta-\tilde{c}\right)}{1+\exp \left(\left(\tau^{2}\right)^{1 / 2} \tilde{c} / \theta-\tilde{c}\right)}\right) .
$$

\section{C.2 Choice of $\tilde{c}$}

Our choice of $\tilde{c}$ is based on the idea that the probability for $\tau$ falling into the interval $[0, \theta]$ is sufficiently close to 1 ,i.e.

$$
\begin{aligned}
& p(\tau \leq \theta) \geq 1-\alpha^{*} \\
& \Longleftrightarrow \alpha^{*} \geq \frac{\tilde{c}^{-1} \log (2)}{1+\tilde{c}^{-1} \log (1+\exp (-\tilde{c}))}
\end{aligned}
$$

Hence, for given $\alpha^{*}$ we can choose $\tilde{c}$ as the root of the function

$$
r(\tilde{c})=\frac{\tilde{c}^{-1} \log (2)}{1+\tilde{c}^{-1} \log (1+\exp (-\tilde{c}))}-\alpha^{*} .
$$

Based on several choices for $\alpha^{*}$, we set $\alpha^{*}=0.05$ as the default value. The density for $\tau^{2}$ as a function of $\tilde{c}$ and $\theta$ is implemented in our R-package sdPrior.

\section{C.3 Cumulative Distribution Function of $\tau^{2}$}

Random number generation of $\tau^{2}$ with density (C.2) is realised with the inversion method based on the cumulative distribution function and implemented in our Rpackage sdPrior. The cumulative distribution is simply obtained as

$$
F\left(\tau^{2}\right)=\int_{0}^{\tau^{2}} p(x) \mathrm{d} x
$$

with $p\left(\tau^{2}\right)$ defined in (C.2). Some calculations yield

$$
\begin{aligned}
F\left(\tau^{2}\right) & =\frac{\theta^{-1}}{1+\log (1+\exp (-\tilde{c})) / \tilde{c}}\left[x^{1 / 2}-\frac{\theta}{\tilde{c}} \log \left(1+\exp \left(x^{1 / 2} \tilde{c} / \theta-\tilde{c}\right)\right)\right]_{0}^{\tau^{2}} \\
& =\frac{1}{\tilde{c}+\log (1+\exp (-\tilde{c}))}\left[\frac{\tilde{c}}{\theta}\left(\tau^{2}\right)^{1 / 2}-\log \left(\exp \left(\left(\tau^{2}\right)^{1 / 2} \tilde{c} / \theta\right)+\exp (\tilde{c})\right)\right]+1
\end{aligned}
$$

where for the last equality

$$
\frac{1}{1+\log (1+\exp (-\tilde{c})) / \tilde{c}}=\frac{\tilde{c}}{\tilde{c}+\log (1+\exp (-\tilde{c}))}
$$

and

$$
\log \left(1+\exp \left(\left(\tau^{2}\right)^{1 / 2} \tilde{c} / \theta-\tilde{c}\right)\right)=\log \left(\exp \left(\left(\tau^{2}\right)^{1 / 2} \tilde{c} / \theta\right)+\exp (\tilde{c})\right)-\tilde{c}
$$

have been used. 


\section{Densities for $\tau^{2}$ with Half-Normal and Half- Cauchy Prior for $\tau$}

\section{D.1 Half-Normal Prior for $\tau$}

The density of $\tau$ in case of a half-normal prior with location parameter 0 and scale parameter $\theta>0$ is given by

$$
p(\tau)=\theta^{-1} \sqrt{2 / \pi} \exp \left(-\frac{\tau^{2}}{2 \theta^{2}}\right) .
$$

Applying the change of variable theorem to the transformation $g(\tau)=\tau^{2}$ yields

$$
p\left(\tau^{2}\right)=\frac{\left(\tau^{2}\right)^{1 / 2-1}}{\sqrt{2 \pi \theta^{2}}} \exp \left(-\frac{\tau^{2}}{2 \theta^{2}}\right)
$$

which is a gamma distribution with parameters $a=1 / 2, b=\left(2 \theta^{2}\right)^{-1}$ and density proportional to $p(x) \propto x^{a-1} \exp (-b x), x \geq 0$.

\section{D.2 Half-Cauchy Prior for $\tau$}

The density of $\tau$ in case of a half-Cauchy prior with location parameter 0 and scale parameter $\theta>0$ is given by

$$
p(\tau)=\frac{2}{\pi}\left(1+(\tau / \theta)^{2}\right)^{-1}
$$

Applying the change of variable theorem to the transformation $g(\tau)=\tau^{2}$ yields

$$
p\left(\tau^{2}\right)=\frac{1}{\pi \theta^{2}}\left(1+\frac{\tau^{2}}{\theta^{2}}\right)^{-1}\left(\frac{\tau^{2}}{\theta^{2}}\right)^{-1 / 2}
$$

which is a generalised beta prime distribution with parameters $a=1 / 2, b=1 / 2$, $t=1, q=\theta^{2}$ and density

$$
p(x)=\frac{t(x / q)^{a p-1}\left(1+(x / q)^{t}\right)^{-a-b}}{q \mathrm{~B}(a, b)},
$$

where $\mathrm{B}(a, b)=\pi$ denotes the beta function of $a, b$. 


\section{E Densities on the Distance Scale for the Alterna- tive Hyperprior Structures}

From the proof of Theorem 1 we know that

$$
-\lambda d\left(\tau^{2}\right) \stackrel{\tau_{b}^{2} \rightarrow 0}{\rightarrow}-\left(\frac{\tau^{2}}{\theta}\right)^{1 / 2}
$$

holds with

$$
\theta^{-1 / 2}=\lambda \sqrt{\frac{\kappa}{\tau_{b}^{2}}}
$$

and $\lambda$ such that $\theta^{-1 / 2}$ is kept constant for $\tau_{b}^{2} \rightarrow 0$. Hence, the transformation $d=g\left(\tau^{2}\right)$ is proportional to $\left(\tau^{2}\right)^{1 / 2}$ which means that for alternative hyperpriors defined for $\tau$ we obtain the original densities for the priors on the distance scale $d\left(\tau^{2}\right)$.

\section{E.1 Inverse Gamma Prior for $\tau^{2}$}

The prior for $\tau^{2}$ is an inverse gamma distribution with density

$$
p(x)=\frac{\theta^{a}}{\Gamma(a)} x^{-a-1} \exp \left(-\frac{\theta}{x}\right), \quad x>0,
$$

shape parameter $a>0$, scale parameter $\theta>0$ and $\Gamma(a)$ the gamma function of $a$. Hence, the prior for $d\left(\tau^{2}\right)$ is up to a constant given by

$$
p(d) \propto d^{-2 a-1} \exp \left(-\frac{1}{\lambda^{2} d^{2}}\right) .
$$

In particular, for $a=1$ we obtain

$$
p(d)=\frac{2}{\lambda^{2} d^{3}} \exp \left(-\frac{1}{\lambda^{2} d^{2}}\right) .
$$

\section{E.2 Half-Normal Prior for $\tau$}

Since the prior for $\tau^{2}$ is a gamma distribution with shape parameter $a=1 / 2$ and $b=\left(2 \theta^{2}\right)^{-1}$, we obtain

$$
p(d)=\frac{\lambda}{\sqrt{2 \pi} \theta} \exp \left(-\frac{\lambda^{2}}{2 \theta} d^{2}\right)
$$

which is a half-normal distribution with location parameter 0 and scale parameter $\sqrt{\theta} / \lambda$.

\section{E.3 Half-Cauchy Prior for $\tau$}

Since the prior for $\tau^{2}$ is a generalised beta prime distribution with parameters $a=1 / 2$, $b=1 / 2, t=1, q=\theta^{2}$, we obtain

$$
p(d)=\frac{2 \sqrt{\theta}}{\pi \lambda}\left(1+\frac{\lambda^{2} d^{2}}{\theta}\right)^{-1}
$$

which is a half-Cauchy prior with location parameter 0 and scale parameter $\sqrt{\theta} / \lambda$. 


\section{E.4 Approximate Uniform Prior for $\tau$}

Due to the density (C.2) with scale parameter $\theta$ for $\tau^{2}$ we obtain

$$
p(d)=\frac{(\sqrt{\theta} / \lambda)^{-1}}{1+\tilde{c}^{-1} \log (1+\exp (\tilde{c}))}\left(1-\frac{\exp \left(\frac{\tau \tilde{c}}{\sqrt{\theta} / \lambda}-\tilde{c}\right)}{1+\exp \left(\frac{\tau \tilde{c}}{\sqrt{\theta} / \lambda}-\tilde{c}\right)}\right)
$$

which is a density of the form (C.1) with scale parameter $\sqrt{\theta} / \lambda$ and $\tilde{c}$ from Section C.2. 


\section{F Details on MCMC Inference and IWLS Propos- als}

\section{F.1 Derivations of Proposal Densities}

In this section, we derive explicit formulae for the parameters $\mu_{u}$ and $\sigma_{u}^{2}$ of the proposal densities $\mathrm{N}\left(\mu_{u}, \sigma_{u}^{2}\right)$ for scale-dependent priors for $\tau^{2}$ as well as the alternative priors from Sections $\mathrm{C}$ and $\mathrm{D}$. The strategy is to follow the structure of Section 4.1.2 (dropping the indices $j, k$ ) and to plug in the appropriate prior densities $p\left(\tau^{2}\right)$. In particular, we construct the proposal density for $g\left(\tau^{2}\right)=\log \left(\tau^{2}\right)=u$ in all cases to obtain a transformed variable with values on the complete real line. This is reasonable to avoid invalid proposed values, see also Section F.2. For notational convenience, recall that the distribution of $u$ is obtained with the change of variable theorem as

$$
p(u)=p_{\tau^{2}}\left(g^{-1}(u)\right)\left|\left(g^{-1}(u)\right)^{\prime}\right| .
$$

Hence, the full conditional for $u$ is (up to multiplicative constants not depending on u)

$$
p(u \mid \cdot) \propto p\left(\boldsymbol{\beta} \mid g^{-1}(u)\right) p_{\tau^{2}}\left(g^{-1}(u)\right)\left|\left(g^{-1}(u)\right)^{\prime}\right|
$$

with $\tau^{2}=g^{-1}(u)=\exp (u),\left(g^{-1}(u)\right)^{\prime}=\partial g^{-1}(u) / \partial u=\exp (u)$ and $p_{\tau^{2}}\left(g^{-1}(u)\right)$ the density of $\tau^{2}$ with $g^{-1}(u)$ plugged in. Hence, the log-full conditional is (up to additive constants not depending on $u$ ) given by

$$
l_{u} \equiv \log (p(u \mid \cdot)) \propto \frac{u}{2}(2-\operatorname{rk}(\boldsymbol{K}))-\frac{1}{2 \exp (u)} \boldsymbol{\beta}^{\prime} \boldsymbol{K} \boldsymbol{\beta}+\log \left(p_{\tau^{2}}\left(g^{-1}(u)\right)\right) .
$$

According to Section 4.1.2 we have to compute the first and second derivatives $\frac{\partial l_{u}}{\partial u}$, $\frac{\partial^{2} l_{u}}{\partial u^{2}}$ to obtain the required parameters $\mu_{u}, \sigma_{u}^{2}$

$$
\mu_{u}=\sigma_{u}^{2} \frac{\partial l_{u}}{\partial u}+u \quad \text { and } \quad \sigma_{u}^{2}=-1 / \frac{\partial^{2} l_{u}}{\partial u^{2}}
$$

\section{F.1.1 Scale-Dependent Prior for $\tau^{2}$}

With scale-dependent prior for $\tau^{2}$ (Weibull distribution with density given in (6) ) the density of $u$ is

$$
p(u)=\exp (u)(\exp (u))^{-1 / 2} \exp \left(\left(-\frac{\exp (u)}{\theta}\right)^{1 / 2}\right) .
$$

The full conditional is consequently proportional to

$$
p(u \mid \cdot) \propto \frac{\exp (u)}{(\exp (u))^{\mathrm{rk}(\boldsymbol{K}) / 2}} \exp \left(-\frac{\boldsymbol{\beta}^{\prime} \boldsymbol{K} \boldsymbol{\beta}}{2 \exp (u)}\right)(\exp (u))^{-1 / 2} \exp \left(\left(-\frac{\exp (u)}{\theta}\right)^{1 / 2}\right)
$$

and the log-full conditional is

$$
\log (p(u \mid \cdot)) \propto \frac{1}{2}(1-\operatorname{rk}(\boldsymbol{K})) u-\frac{\boldsymbol{\beta}^{\prime} \boldsymbol{K} \boldsymbol{\beta}}{2 \exp (u)}-\left(\frac{\exp (u)}{\theta}\right)^{1 / 2} .
$$


The first and second derivatives of $\log (p(u \mid \cdot))$ with respect to $u$ are computed as

$$
\begin{aligned}
\frac{\partial l_{u}}{\partial u} & =\frac{1-\operatorname{rk}(\boldsymbol{K})}{2}+\frac{\boldsymbol{\beta}^{\prime} \boldsymbol{K} \boldsymbol{\beta}}{2 \exp (u)}-\left(\frac{\exp (y)}{4 \theta}\right)^{1 / 2} \\
\frac{\partial^{2} l_{u}}{\partial u^{2}} & =-\frac{\boldsymbol{\beta}^{\prime} \boldsymbol{K} \boldsymbol{\beta}}{2 \exp (u)}-\left(\frac{\exp (u)}{16 \theta}\right)^{1 / 2} .
\end{aligned}
$$

\section{F.1.2 Half-Normal Prior for $\tau$}

With half-normal prior for $\tau, \tau^{2}$ has a gamma distribution with density given in Section D.1. Hence, the density of $u$ is

$$
p(u)=\frac{1}{2} \sqrt{\exp (u)} \frac{\sqrt{2}}{\sqrt{\pi} \theta} \exp \left(-\frac{\exp (u)}{2 \theta^{2}}\right) .
$$

The full conditional is consequently proportional to

$$
p(u \mid \cdot) \propto(\exp (u))^{-\operatorname{rk}(\boldsymbol{K}) / 2} \exp \left(-\frac{\boldsymbol{\beta}^{\prime} \boldsymbol{K} \boldsymbol{\beta}}{2 \exp (u)}\right)(\exp (u))^{1 / 2} \exp \left(-\frac{\exp (u)}{2 \theta^{2}}\right)
$$

and the log-full conditional is

$$
\log (p(u \mid \cdot)) \propto-\frac{\operatorname{rk}(\boldsymbol{K})}{2} u-\frac{\boldsymbol{\beta}^{\prime} \boldsymbol{K} \boldsymbol{\beta}}{2 \exp (u)}+\frac{u}{2}-\frac{\exp (u)}{2 \theta^{2}} .
$$

The first and second derivatives of $\log (p(u \mid \cdot))$ with respect to $u$ are computed as

$$
\begin{aligned}
\frac{\partial l_{u}}{\partial u} & =\frac{1}{2}\left(1-\operatorname{rk}(\boldsymbol{K})+\frac{\boldsymbol{\beta}^{\prime} \boldsymbol{K} \boldsymbol{\beta}}{\exp (u)}-\frac{\exp (u)}{\theta^{2}}\right) \\
\frac{\partial^{2} l_{u}}{\partial u^{2}} & =-\frac{1}{2}\left(\frac{\boldsymbol{\beta}^{\prime} \boldsymbol{K} \boldsymbol{\beta}}{\exp (u)}-\frac{\exp (u)}{\theta^{2}}\right) .
\end{aligned}
$$

\section{F.1.3 Half-Cauchy Prior for $\tau$}

With half-Cauchy prior for $\tau, \tau^{2}$ has a generalised beta prime distribution with density given in Section D.2. Hence, the density of $u$ is

$$
p(u)=\frac{1}{\pi} \exp (u)\left(\exp (u) / \theta^{2}\right)^{-1 / 2}\left(1+\exp (u) / \theta^{2}\right)^{-1} .
$$

The full conditional is consequently proportional to

$$
p(u \mid \cdot) \propto(\exp (u))^{-\operatorname{rk}(\boldsymbol{K}) / 2} \exp \left(-\frac{\boldsymbol{\beta}^{\prime} \boldsymbol{K} \boldsymbol{\beta}}{2 \exp (u)}\right)(\exp (u))^{1 / 2} \theta\left(1+\exp (u) / \theta^{2}\right)^{-1}
$$

and the log-full conditional is

$$
\log (p(u \mid \cdot)) \propto-\frac{\operatorname{rk}(\boldsymbol{K})}{2}-\frac{\boldsymbol{\beta}^{\prime} \boldsymbol{K} \boldsymbol{\beta}}{2 \exp (u)}+\frac{u}{2}-\log \left(1+\exp (u) / \theta^{2}\right) .
$$

The first and second derivatives of $\log (p(u \mid \cdot))$ with respect to $u$ are computed as

$$
\begin{aligned}
\frac{\partial l_{u}}{\partial u} & =\frac{1}{2}(1-\operatorname{rk}(\boldsymbol{K}))+\frac{\boldsymbol{\beta}^{\prime} \boldsymbol{K} \boldsymbol{\beta}}{2 \exp (u)}-\frac{\exp (u) / \theta^{2}}{1+\exp (u) / \theta^{2}} \\
\frac{\partial^{2} l_{u}}{\partial u^{2}} & =-\frac{\boldsymbol{\beta}^{\prime} \boldsymbol{K} \boldsymbol{\beta}}{2 \exp (u)}-\frac{\exp (u) / \theta^{2}}{\left(1+\exp (u) / \theta^{2}\right)^{2}} .
\end{aligned}
$$




\section{F.1.4 Approximate Uniform Prior for $\tau$}

With approximate uniform prior for $\tau$ as defined in Section C.1, $\tau^{2}$ has a distribution with density given in (C.2). Hence, the density of $u$ is

$$
\begin{aligned}
p(u) & =\frac{\exp (u)(\exp (u))^{-1 / 2}}{2 \theta\left(1+\frac{1}{\tilde{c}} \log (1+\exp (-\tilde{c}))\right)}\left(1-\frac{\exp \left(\frac{(\exp (u))^{1 / 2} \tilde{c}}{\theta}-\tilde{c}\right)}{1+\exp \left(\frac{(\exp (u))^{1 / 2} \tilde{c}}{\theta}-\tilde{c}\right)}\right) \\
& \propto \frac{\sqrt{\exp (u)}}{\theta\left(1+\exp \left(\frac{\tilde{c} \sqrt{\exp (u)}}{\theta}-\tilde{c}\right)\right)}
\end{aligned}
$$

The full conditional is consequently proportional to

$$
p(u \mid \cdot) \propto(\exp (u))^{-\operatorname{rk}(\boldsymbol{K}) / 2} \exp \left(-\frac{\boldsymbol{\beta}^{\prime} \boldsymbol{K} \boldsymbol{\beta}}{2 \exp (u)}\right) \frac{\sqrt{\exp (u)}}{\theta\left(1+\exp \left(\frac{\tilde{c} \sqrt{\exp (u)}}{\theta}-\tilde{c}\right)\right)}
$$

and the log-full conditional is

$$
\log (p(u \mid \cdot)) \propto-u \frac{\operatorname{rk}(\boldsymbol{K})}{2}-\frac{\boldsymbol{\beta}^{\prime} \boldsymbol{K} \boldsymbol{\beta}}{2 \exp (u)}+\frac{u}{2}-\log \left(1+\exp \left(\frac{\tilde{c} \sqrt{\exp (u)}}{\theta}-\tilde{c}\right)\right) .
$$

The first and second derivatives of $\log (p(u \mid \cdot))$ with respect to $u$ are computed as

$$
\begin{aligned}
\frac{\partial l_{u}}{\partial u} & =\frac{1}{2}-\frac{\operatorname{rk}(\boldsymbol{K})}{2}+\frac{\boldsymbol{\beta}^{\prime} \boldsymbol{K} \boldsymbol{\beta}}{2 \exp (u)}-\frac{\exp \left(\frac{\tilde{c} \sqrt{\exp (u)}}{\theta}-\tilde{c}\right) \frac{\tilde{c} \sqrt{\exp (u)}}{2 \theta}}{1+\exp \left(\frac{\tilde{c} \sqrt{\exp (u)}}{\theta}-\tilde{c}\right)} \\
\frac{\partial^{2} l_{u}}{\partial u^{2}} & =-\frac{\boldsymbol{\beta}^{\prime} \boldsymbol{K} \boldsymbol{\beta}}{2 \exp (u)}-\frac{\exp \left(\frac{\tilde{c} \sqrt{\exp (u)}}{\theta}-\tilde{c}\right) \frac{\tilde{c}^{2} \exp (u)}{4 \theta^{2}}}{\left(1+\exp \left(\frac{\tilde{c} \sqrt{\exp (u)}}{\theta}-\tilde{c}\right)\right)^{2}}-\frac{\exp \left(\frac{\tilde{c} \sqrt{\exp (u)}}{\theta}-\tilde{c}\right) \frac{\tilde{c} \sqrt{\exp (u)}}{4 \theta}}{1+\exp \left(\frac{\tilde{c} \sqrt{\exp (u)}}{\theta}-\tilde{c}\right)} .
\end{aligned}
$$

\section{F.2 Equivalence of Acceptance Probabilities}

In Remark 5 we have mentioned that in distribution $\alpha_{\text {accept }}$ and $\tilde{\alpha}_{\text {accept }}$ are equivalent in the sense that in distribution using the proposal density $q(u)=\mathrm{N}\left(\mu_{u}, \sigma^{2}\right)$ and to back-transform $\tau^{2}=\exp (u)=g^{-1}(u)$ to obtain a current value for $\tau^{2}$ during the MCMC run is in distribution equivalent to constructing a proposal density $\tilde{q}\left(\tau^{2}\right)$ for $\tau^{2}$ for $q$ and to draw a proposal for $\tau^{2}$ from $\tilde{q}$. The reason is as follows: Let as in the previous section $p(u \mid \cdot)$ be the full conditional for $u$, i.e.

$$
p(u \mid \cdot) \propto \frac{\exp (u)}{(\exp (u))^{\mathrm{rk}(\boldsymbol{K}) / 2}} \exp \left(-\frac{\boldsymbol{\beta}^{\prime} \boldsymbol{K} \boldsymbol{\beta}}{2 \exp (u)}\right) p_{\tau^{2}}\left(g^{-1}(u)\right) .
$$

For obtaining the proposal density $q(u)=\mathrm{N}\left(\mu_{u}, \sigma_{u}^{2}\right)$ with parameters given in (F.1) we need to compute the first two derivatives of $l_{u} \equiv \log (p(u \mid \cdot))$ which are

$$
\begin{aligned}
\frac{\partial l_{u}}{\partial u} & =1-\frac{\operatorname{rk}(\boldsymbol{K})}{2}+\frac{\boldsymbol{\beta}^{\prime} \boldsymbol{K} \boldsymbol{\beta}}{2 \exp (u)}+\frac{\partial \log \left(p_{\tau^{2}}\left(g^{-1}(u)\right)\right)}{\partial u} \\
\frac{\partial^{2} l_{u}}{\partial u^{2}} & =-\frac{\boldsymbol{\beta}^{\prime} \boldsymbol{K} \boldsymbol{\beta}}{2 \exp (u)}+\frac{\partial^{2} \log \left(p_{\tau^{2}}\left(g^{-1}(u)\right)\right)}{\partial u^{2}} .
\end{aligned}
$$


Let now $u$ be the proposal and $u^{(c)}=\log \left(\left(\tau^{2}\right)^{(c)}\right)$ the current state. Then the acceptance probability $\alpha_{\text {accept }}$ is given by

$$
\alpha_{\text {accept }}=\frac{p(u \mid \cdot) q\left(u^{(c)} \mid y\right)}{p\left(u^{(c)} \mid \cdot\right) q\left(u \mid u^{(c)}\right)}
$$

and

$$
\begin{aligned}
\log \left(\alpha_{\text {accept }}\right) \propto & (1-\operatorname{rk}(\boldsymbol{K}) / 2)\left(u-u^{(c)}\right)-\frac{\boldsymbol{\beta}^{\prime} \boldsymbol{K} \boldsymbol{\beta}}{2}\left(\frac{1}{\exp (u)}-\frac{1}{\exp \left(u^{(c)}\right)}\right) \\
& -\left(\frac{\partial \log \left(p_{\tau^{2}}\left(g^{-1}(u)\right)\right)}{\partial u}-\frac{\partial \log \left(p_{\tau^{2}}\left(g^{-1}\left(u^{(c)}\right)\right)\right)}{\partial u^{(c)}}\right) \\
& -\frac{1}{2}\left(\sigma_{u}^{2}-\left(\sigma_{u}^{2}\right)^{(c)}\right)-\frac{1}{2}\left(\left(\frac{u^{(c)}-\mu_{u}}{\sigma_{u}^{2}}\right)^{2}-\left(\frac{u-\mu_{u}}{\sigma_{u}^{2}}\right)^{2}\right) .
\end{aligned}
$$

$\tilde{q}\left(\tau^{2}\right)$ for $\tau^{2}$ can be obtained using the change of variable theorem with the backtransformation $\tau^{2}=\exp (u), g\left(\tau^{2}\right)=\log \left(\tau^{2}\right)$ and $\left(g\left(\tau^{2}\right)\right)^{\prime}=\tau^{-2}$ such that $\tilde{q}$ results in a lognormal distribution

$$
\tilde{q}\left(\tau^{2}\right)=\frac{1}{\tau^{2} \sqrt{2 \pi \sigma_{u}^{2}}} \exp \left(-\frac{1}{2}\left(\frac{\log \left(\tau^{2}\right)-\mu_{u}}{\sigma_{u}}\right)^{2}\right)
$$

with parameters $\mu_{u}, \sigma_{u}^{2}$

$$
\mu_{u}=\sigma_{u}^{2} \frac{\partial l_{g\left(\tau^{2}\right)}}{\partial g\left(\tau^{2}\right)}+g\left(\tau^{2}\right) \quad \text { and } \quad \sigma_{u}^{2}=-1 / \frac{\partial^{2} l_{g\left(\tau^{2}\right)}}{\partial\left(g\left(\tau^{2}\right)\right)^{2}}
$$

and derivatives

$$
\begin{aligned}
\frac{\partial l_{g\left(\tau^{2}\right)}}{\partial g\left(\tau^{2}\right)} & =1-\frac{\operatorname{rk}(\boldsymbol{K})}{2}+\frac{\boldsymbol{\beta}^{\prime} \boldsymbol{K} \boldsymbol{\beta}}{2 \tau^{2}}+\frac{\partial \log \left(p_{\tau^{2}}\left(\tau^{2}\right)\right)}{\partial g\left(\tau^{2}\right)} \\
\frac{\partial^{2} l_{g\left(\tau^{2}\right)}}{\partial\left(g\left(\tau^{2}\right)\right)^{2}} & =-\frac{\boldsymbol{\beta}^{\prime} \boldsymbol{K} \boldsymbol{\beta}}{2 \tau^{2}}+\frac{\partial^{2} \log \left(p_{\tau^{2}}\left(\tau^{2}\right)\right)}{\partial\left(g\left(\tau^{2}\right)\right)^{2}}
\end{aligned}
$$

which are in fact in distribution equivalent to $\frac{\partial l_{u}}{\partial u}$ and $\frac{\partial^{2} l_{u}}{\partial u^{2}}$ above. Hence, in distribution the parameters $\mu_{u}$ and $\sigma_{u}^{2}$ are equivalent in both variants. Furthermore, for the acceptance probability $\tilde{\alpha}_{\text {accept }}$ we have

$$
\tilde{\alpha}_{\text {accept }}=\frac{p\left(\tau^{2} \mid \cdot\right) q\left(\left(\tau^{2}\right)^{(c)} \mid \tau^{2}\right)}{p\left(\left(\tau^{2}\right)^{(c)} \mid \cdot\right) q\left(\tau^{2} \mid\left(\tau^{2}\right)^{(c)}\right)}
$$

and

$$
\begin{aligned}
\log \left(\tilde{\alpha}_{\text {accept }}\right) \propto & (1-\operatorname{rk}(\boldsymbol{K}) / 2)\left(\log \left(\tau^{2}\right)-\log \left(\left(\tau^{2}\right)^{(c)}\right)\right)-\frac{\boldsymbol{\beta}^{\prime} \boldsymbol{K} \boldsymbol{\beta}}{2}\left(\frac{1}{\tau^{2}}-\frac{1}{\left(\tau^{2}\right)^{(c)}}\right) \\
& -\left(\frac{\partial \log \left(p_{\tau^{2}}\left(\tau^{2}\right)\right)}{\partial g\left(\tau^{2}\right)}-\frac{\partial \log \left(p_{\tau^{2}}\left(\left(\tau^{2}\right)^{(c)}\right)\right.}{\partial\left(g\left(\tau^{2}\right)\right)^{(c)}}\right) \\
& -\frac{1}{2}\left(\sigma_{u}^{2}-\left(\sigma_{u}^{2}\right)^{(c)}\right)-\frac{1}{2}\left(\left(\frac{\left(\log \left(\tau^{2}\right)\right)^{(c)}-\mu_{u}}{\sigma_{u}^{2}}\right)^{2}-\left(\frac{\log \left(\tau^{2}\right)-\mu_{u}}{\sigma_{u}^{2}}\right)^{2}\right) .
\end{aligned}
$$

Hence, $\alpha_{\text {accept }}$ and $\tilde{\alpha}_{\text {accept }}$ are equivalent in distribution and even the same when random number generation of $\tilde{q}$ is to draw a random number from the corresponding normal distribution and then to take the logarithm. 


\section{F.3 Validity of IWLS Proposals}

Proposition 3. Assume that

(d.1) $\boldsymbol{\beta}^{\prime} \boldsymbol{K} \boldsymbol{\beta}>0 \forall \boldsymbol{\beta} \in \mathbb{R}^{D}$

(d.2) $\theta>0$.

hold. Let $\mathrm{N}\left(\mu_{u}, \sigma_{u}^{2}\right)$ be one of the proposal densities resulting from the general derivation in Section 4.1.2 and with parameters $\mu_{u}, \sigma_{u}^{2}$ from Section F.1. Then, the proposal density has positive variance, i.e.

$$
\sigma_{u}^{2}>0
$$

holds such that the proposal density is well-defined.

Proof. What needs to be done is to check that the negative second derivatives $-\frac{\partial^{2} l_{u}}{\partial u^{2}}$ are positive for all $u \in \mathbb{R}$. In particular, we need to show that

(i)

$$
g_{1}(u)=\frac{\boldsymbol{\beta}^{\prime} \boldsymbol{K} \boldsymbol{\beta}}{2 \exp (u)}+\left(\frac{\exp (u)}{16 \theta}\right)^{1 / 2}
$$

(ii)

$$
g_{2}(u)=\frac{1}{2}\left(\frac{\boldsymbol{\beta}^{\prime} \boldsymbol{K} \boldsymbol{\beta}}{\exp (u)}+\frac{\exp (u)}{\theta^{2}}\right)
$$

(iii)

$$
g_{3}(u)=\frac{\boldsymbol{\beta}^{\prime} \boldsymbol{K} \boldsymbol{\beta}}{2 \exp (u)}+\frac{\exp (u) / \theta^{2}}{\left(1+\exp (u) / \theta^{2}\right)^{2}}
$$

$$
g_{4}(u)=\frac{\boldsymbol{\beta}^{\prime} \boldsymbol{K} \boldsymbol{\beta}}{2 \exp (u)}+\frac{\exp \left(\frac{\tilde{c} \sqrt{\exp (u)}}{\theta}-\tilde{c}\right) \frac{\tilde{c}^{2} \exp (u)}{4 \theta^{2}}}{\left(1+\exp \left(\frac{\tilde{c} \sqrt{\exp (u)}}{\theta}-\tilde{c}\right)\right)^{2}}+\frac{\exp \left(\frac{\tilde{c} \sqrt{\exp (u)}}{\theta}-\tilde{c}\right) \frac{\tilde{c} \sqrt{\exp (u)}}{4 \theta}}{1+\exp \left(\frac{\tilde{c} \sqrt{\exp (u)}}{\theta}-\tilde{c}\right)}
$$

are positive. With assumption (d.1) and $\exp (u)>0$ we always have $\frac{\boldsymbol{\beta}^{\prime} \boldsymbol{K} \boldsymbol{\beta}}{2 \exp (u)}>0$. Furthermore, due to (d.2) and the fact that $\exp (f(u))>0$ for any transformation $f$ of $u$, the positivity of $g_{1}, g_{2}, g_{3}$ and $g_{4}$ follows immediately.

Alternative Strategy of the Proof A more general approach for the proof that can be generalised to other prior structures works as follows: We initially show that $l_{u}$ is strictly concave by proofing that $\log \left(\boldsymbol{\beta} \mid g^{-1}(u)\right)$ is strictly concave and $\log (p(u))$ is concave. From 1. we then have $\frac{\partial^{2} l_{u}}{\partial u^{2}} \leq 0 \forall u \in D$. Finally, we show that the assumption $\frac{\partial^{2} l_{u}}{\partial u^{2}}=0$ for some $u \in D$ leads to a contradiction since in general $\partial^{2} \log \left(\boldsymbol{\beta} \mid g^{-1}(u)\right) / \partial u^{2}<0$ holds and specifically for the considered priors also $\partial^{2} \log (p(u)) / \partial u^{2} \leq 0$. Alternatively (and probably more direct but less general), we could simply check that the negative second derivatives $-\frac{\partial^{2} l_{u}}{\partial u^{2}}$ are positive for all $u \in \mathbb{R}$. 


\section{F.4 Algorithm}

In summary, the posterior distribution is assessed as follows (after specifying appropriate values for thinning and burn in phase):

1: Initialise $\boldsymbol{\beta}_{0, k}^{(0)}, \boldsymbol{\beta}_{j, k}^{(0)},\left(\tau_{j, k}^{2}\right)^{(0)}, j=1, \ldots, J_{k}, k=1, \ldots, K$.

2: for $t=1, \ldots, T$ do

3: $\quad$ for $k=1, \ldots, K$ do

4: $\quad$ Update $\boldsymbol{\beta}_{0, k}^{(t)}$ via IWLS (with $\boldsymbol{K}=\mathbf{0}$ ) as specified in Section 4.1.1.

5: $\quad$ for $j=1, \ldots, J_{k}$ do

6: $\quad$ Update $\boldsymbol{\beta}_{j, k}^{(t)}$ via IWLS as specified in Section 4.1.1.

7: $\quad$ Perform the updating step for $\left(\tau_{j, k}^{2}\right)^{(t)}$ :

8: $\quad$ Set $u^{(c)}=\log \left(\left(\tau_{j, k}^{2}\right)^{(t)}\right)$ and

9: $\quad$ compute $\mu_{u}^{(c)},\left(\sigma_{u}^{2}\right)^{(c)}$ according to Sections 4.1.2 or 4.1.3.

10: $\quad$ Draw $u^{(p)} \sim \mathrm{N}\left(\mu_{u}^{(c)},\left(\sigma_{u}^{2}\right)^{(c)}\right)$

11: $\quad$ Draw $r \sim \mathrm{U}(0,1)$

12: $\quad$ if $r \leq \alpha_{\text {accept }}$ with $\alpha_{\text {accept }}$ from (20) then

13: $\quad$ Set $\left(\tau_{j, k}^{2}\right)^{(t+1)}=\exp \left(u^{(p)}\right)$

14: $\quad \operatorname{else}\left(\tau_{j, k}^{2}\right)^{(t+1)}=\left(\tau_{j, k}^{2}\right)^{(t)}$. 


\section{G Simulation Based Comparison with Alternative Hyperpriors}

As summarised in Section 5 of the main paper, we focus on simulations with nonlinear effects of continuous covariates and compare the performance of scale-dependent priors and alternative prior specifications for the smoothing variances in three relevant scenarios. The first scenario in Section G.1 represents a situation in which the true effect is close to the base model, i.e. close to a linear effect. In the second scenario in Section G.2 we investigate the numerical stability of both priors in a situation with a flat likelihood. The third scenario in Section G.3 is an example when the effect has a strongly nonlinear impact on the response variable. In all scenarios, we use the following settings:

- Sample sizes $n$ are chosen from $\{50,100,250,500,1000\}$.

- Covariates are restricted to one single scalar covariate $x \sim \mathrm{U}(-1,1)$.

- Simulated effects visualised in Figure 2 are

Scenario $1 f(x)=\sin (x)$.

Scenario $2 f(x)=7 \exp (-\exp (5 x))$.

Scenario $3 f(x)=1.5 \sin (1.25 \pi x+0.5) / \exp (x)$.

- Responses

$-y \sim \mathrm{N}(f(x), 1)$ in Scenario 1 and 3 .

- $y \sim \operatorname{Be}(\pi), \pi=\exp (f(x)) /(1+\exp (f(x)))$ in Scenario 2.

- Hyperprior specifications

- $(\epsilon, \epsilon)$-inverse gamma priors, $\operatorname{IG}(\epsilon, \epsilon)$ with $\epsilon \in\{0.001,0.01\}$ for $\tau^{2}$.

- IG $(1, \epsilon), \epsilon=0.005$ as used frequently in the literature as well as $\operatorname{IG}(1, \theta)$ (denoted as $\operatorname{IG}(\alpha)$ in the following) for $\tau^{2}$.

- Flat priors for standard deviations $\tau$ and variances $\tau^{2}$.

- Half-normal $(\mathrm{HN}(\alpha))$, half-Cauchy $(\mathrm{HC}(\alpha))$ and approximate uniform $(\mathrm{U}(\alpha))$ prior with scale parameters $\theta$ for $\tau$.

- Scale-dependent priors $(\operatorname{SD}(\alpha))$ with scale parameters $\theta$ for $\tau^{2}$.

- Scale parameters $\theta$ are computed with fixed values $P=1, c=3$ and sequence $\alpha$ in $\{0.01,0.05,0.1,0.2,0.5,0.7\}$.

For each scenario, $R=1000$ replications are used to compute mean squared errors (MSEs), bias, pointwise $95 \%$ credible intervals and corresponding coverage rates.

\section{G.1 Scenario 1: Close to the Base Model}

Logarithmic MSEs and widths of pointwise credible intervals for a $95 \%$ level have been shown in the main paper Figures 3 and 4 . Corresponding coverage rates are given in Figures G10 and G11. Figures G12 and G14 depict boxplots of bias as well as posterior mean, $2.5 \%$ and $97.5 \%$ estimates for different replications. The replications are chosen to match the $2.5 \%, 10 \%, 50 \%, 90 \%$ and $97.5 \%$ best MSE of all replications. Results are summarised in the following.

(1) MSEs. On average, the MSEs are more robust with different scale parameters $\theta$ for the scale-dependent priors as compared to inverse gamma and flat priors. In addition, Figure 3 indicates that MSEs are slightly smaller with scale-dependent priors even when the sample size is increasing. In general, $\operatorname{IG}(\alpha)$ priors have highest MSEs while half-normal and uniform priors for $\tau$ perform comparably well with scale-dependent 


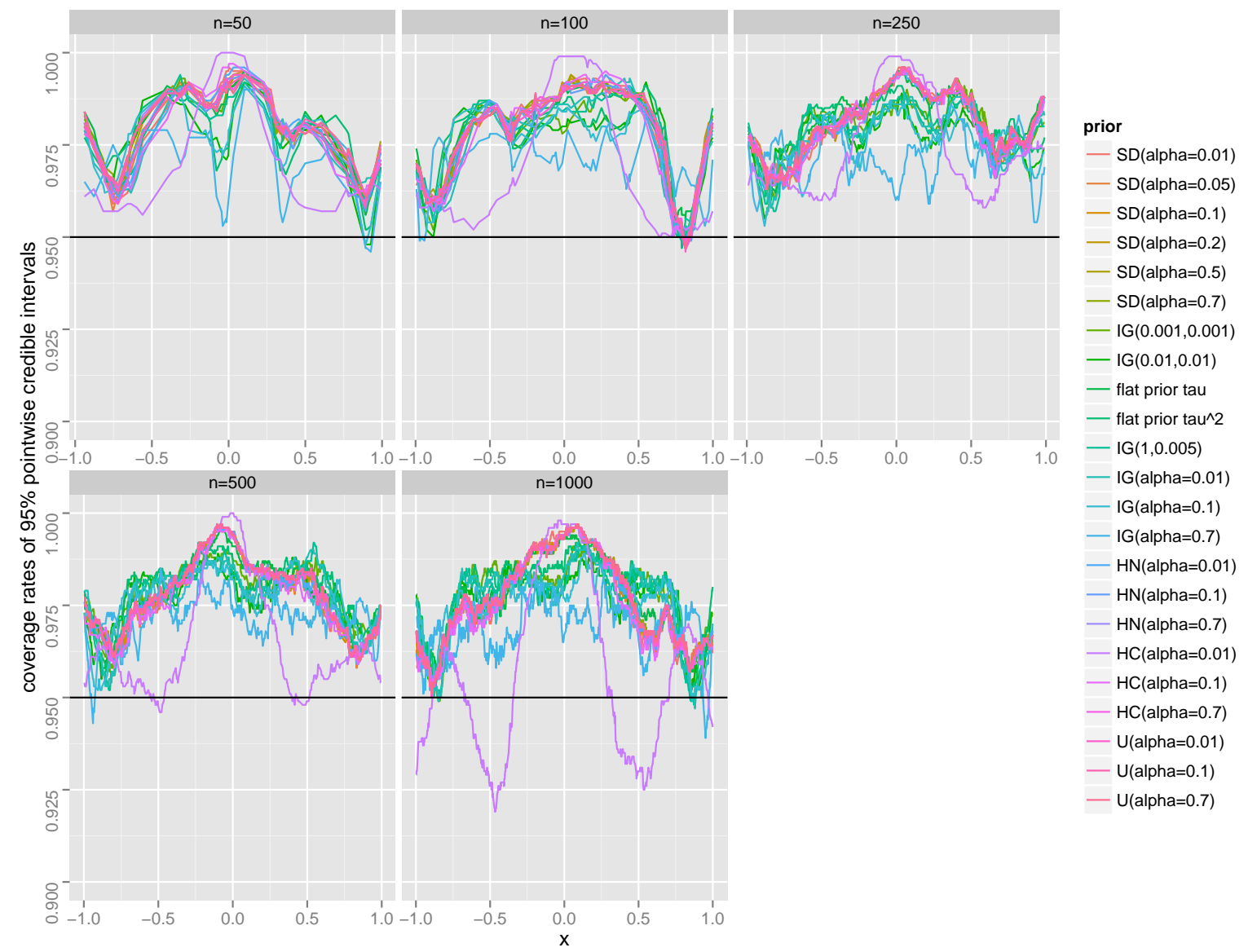

Figure G10: Empirical Evaluation (Scenario 1). Shown are coverage rates of 95\% pointwise credible intervals for different sample sizes $n$ and the different prior settings.

priors in terms of MSEs. Half-Cauchy priors for $\tau$ induce smaller MSEs for $\alpha=0.01$ and similar ones as scale-dependent priors for the remaining values of $\alpha$. Overall, MSEs of scale-dependent, half-normal and uniform priors is very robust with respect to $\alpha$ in this setting.

(2) Widths of credible intervals. Being close to the base model in this setting, the scale-dependent prior outperforms the inverse gamma priors with respect to widths of pointwise credible intervals as shown in Figure 4. One explanation is that the inverse gamma prior tends to estimate too wiggly functions in our setting while the scale-dependent prior benefits from penalisation towards the base model. Again, the scale-dependent priors yield results which are very robust across the different scale parameters $\theta$. In comparison with the remaining alternative priors, credible intervals fit to the behaviour found for MSEs.

(3) Coverage rates. Positively, the narrower credible intervals of scale-dependent priors still maintain the desired coverage level as can be seen in Figures G10, G11, For all priors despite half-Cauchy ones, the coverage rates indicate a slightly too conservative behaviour which frequently occurs when using MCMC simulations. For half-Cauchy priors we see that the partly smallest MSEs and widths of pointwise credible intervals may result in situations where these priors estimate too narrow credible intervals. Hence, the apparently good performance is at least partly offset by this observation. 


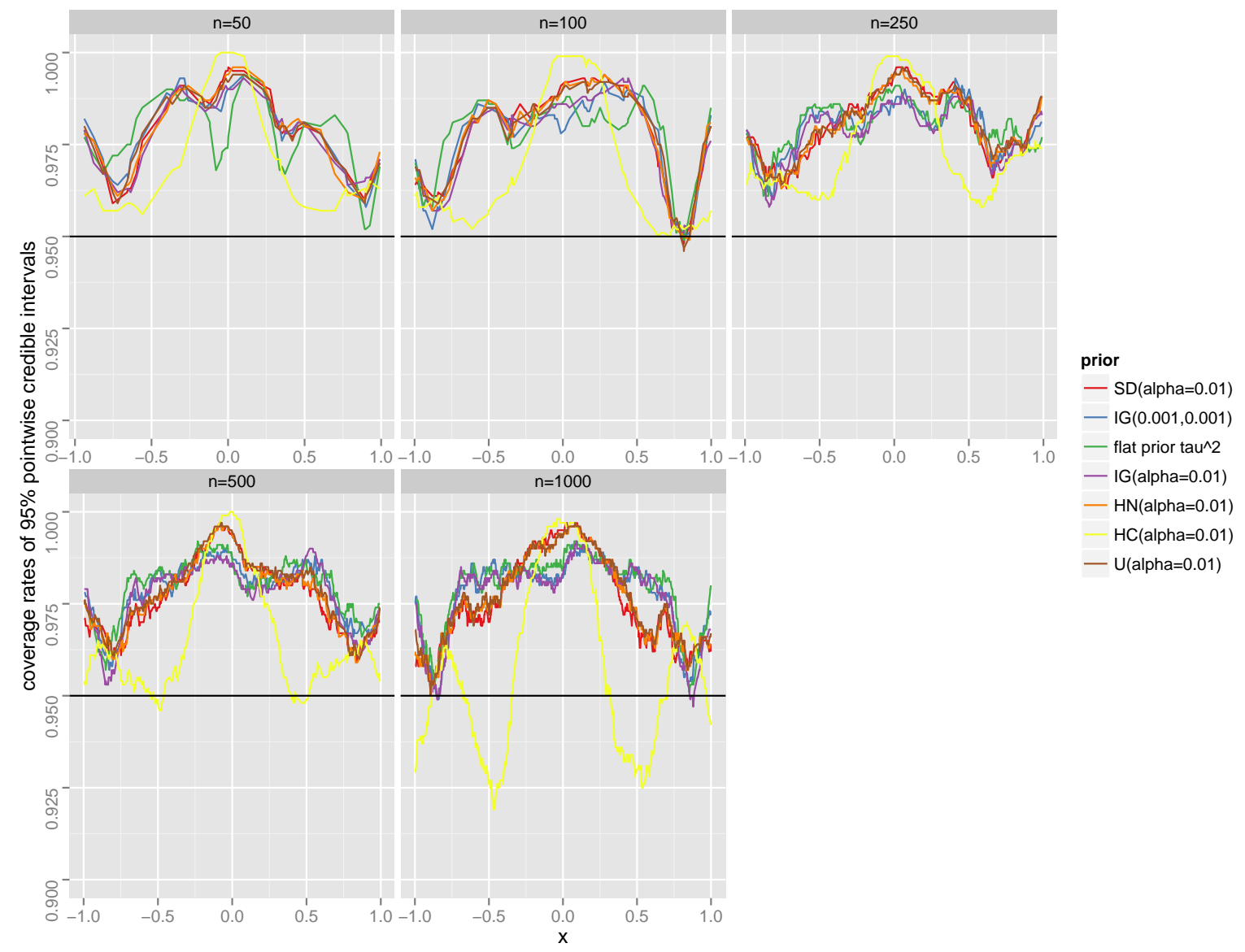

Figure G11: Empirical Evaluation (Scenario 1). Shown are coverage rates of 95\% pointwise credible intervals for different sample sizes $n$ and selected prior settings.

(4) Bias. The bias in this setting is negligibly small for all settings and very similar with all priors.

(5) Selected replications. As expected, Figures G13 and G14 reflect the improvement for estimating the true underlying function (plotted with a solid black line) with increasing sample size.

(6) Flat vs. weakly informative inverse gamma priors. All figures for this simulation indicate a worse performance of flat priors for $\tau$ or $\tau^{2}$ compared to $\operatorname{IG}(\epsilon, \epsilon)$ priors. $\mathrm{IG}(\alpha)$ priors seem to be the worst option in situations close to the base model.

\section{G.2 Scenario 2: Flat Likelihood}

Logarithmic MSEs have been shown in Figure 5 of the main paper. Widths of pointwise credible intervals at a 95\% level are visualised in Figure G15. Corresponding coverage rates are given in Figures G16 and G17. Figures G18 to G20 depict boxplots of bias as well as posterior mean, $2.5 \%$ and $97.5 \%$ estimates for different replications. The replications are chosen to match the $2.5 \%, 10 \%, 50 \%, 90 \%$ and $97.5 \%$ best MSE of all replications.

(1) MSEs. In general, the MSEs in Figure 5 suggest that a moderate number of observations is required to get reasonable estimates in this setting. Still, the scaledependent priors yield smaller MSEs as compared to the $\operatorname{IG}(\varepsilon, \varepsilon)$ priors or flat priors 


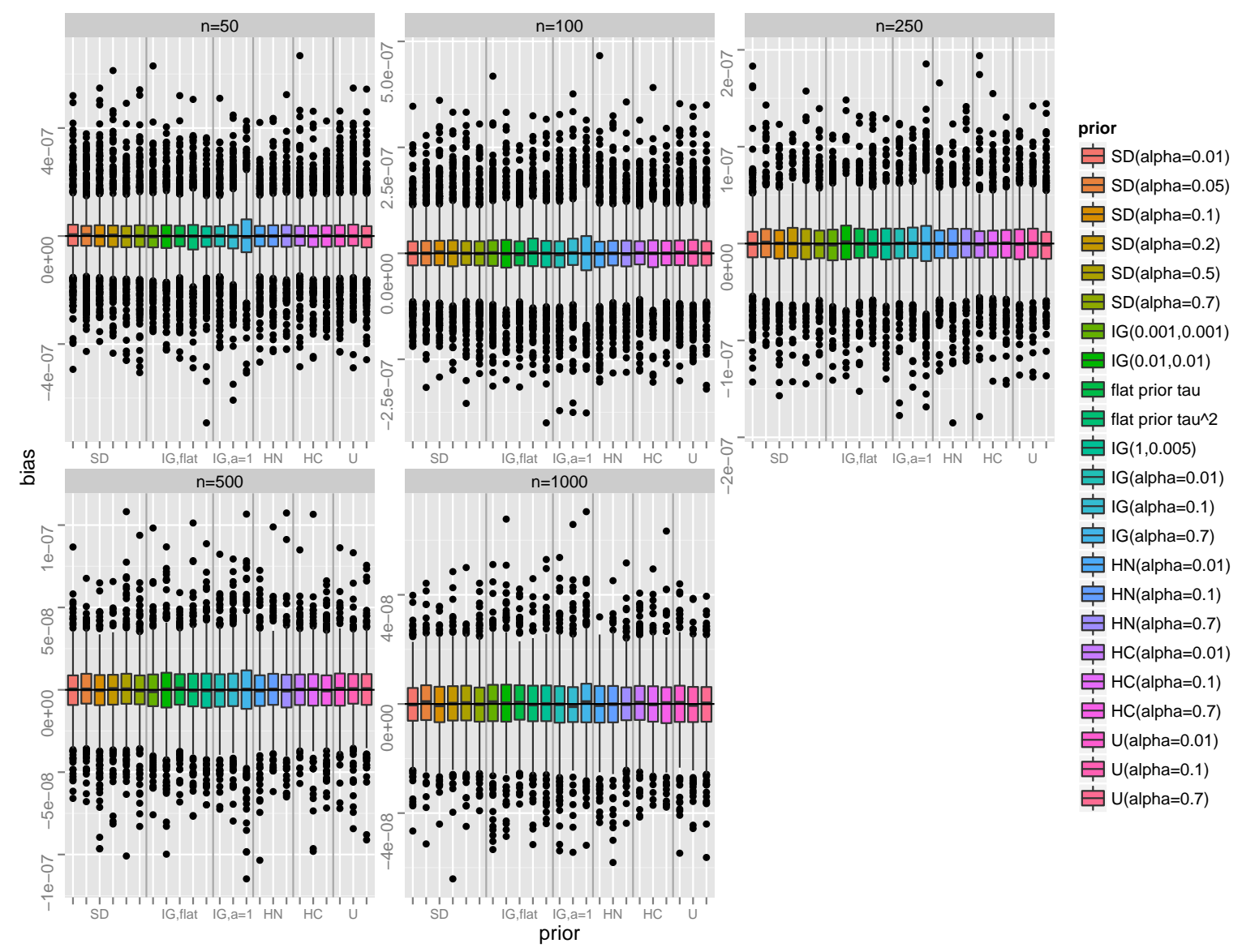

Figure G12: Empirical Evaluation (Scenario 1). Shown are boxplots of bias for different sample sizes $n$ and the different prior settings.

for standard deviations and variances at least when the data sets are small. In contrast to the first scenario, $\operatorname{IG}(\alpha)$ priors perform similar to scale-dependent, halfnormal, half-Cauchy and uniform priors in terms of MSEs.

(2) Widths of credible intervals. In line with the MSEs, credible intervals are extremely wide for $n \leq 250$ supporting the need of moderate sample sizes to estimate such an extreme scenario appropriately well. For all considered sample sizes $n$ however, flat priors for $\tau$ and $\tau^{2}$ perform worst.

(3) Coverage rates. The estimation problems are also reflected in the coverage rates with nominal $95 \%$ level. Scale-dependent priors yield better coverage rates as compared to half-normal, half-Cauchy and uniform priors.

(4) Numerical instabilities. Except for $\operatorname{IG}(\epsilon, \epsilon)$ and flat priors, numerical instabilities occur in less than $1 \%$ of the replications despite the quite extreme data situation with a flat likelihood. Software crashes are observed most frequently with flat priors (up to $26 \%$ ).

\section{G.3 Scenario 3: Strongly Nonlinear Effect}

Logarithmic MSEs and widths of pointwise credible intervals at a 95\% level are visualised in Figures G21 and G22. Corresponding coverage rates are given in Figures G23 and G24. Figures G25 to G27 depict boxplots of bias as well as posterior mean, 2.5\% 


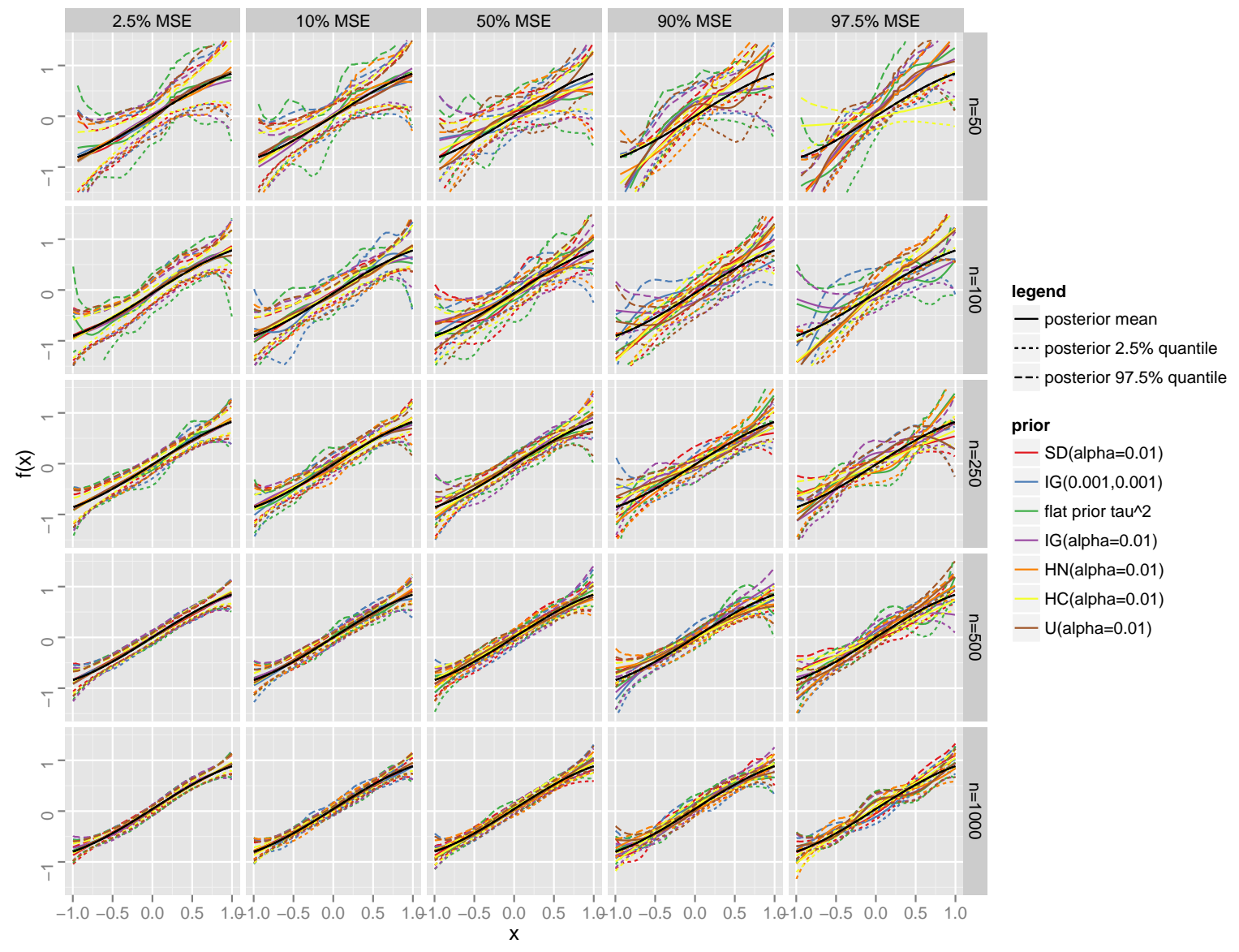

Figure G13: Empirical Evaluation (Scenario 1). Shown are posterior mean, 2.5\% and $97.5 \%$ estimates for selected replications of different sample sizes $n$ and the different prior settings. The replications are chosen to match the $2.5 \%, 10 \%, 50 \%, 90 \%$ and $97.5 \%$ best MSE of the corresponding 1000 replications. The true function is given by the solid black line.

and $97.5 \%$ estimates for different replications. The replications are chosen to match the $2.5 \%, 10 \%, 50 \%, 90 \%$ and $97.5 \%$ best MSE of all replications.

(1) MSEs. Compared to Scenario 1, where MSEs with scale-dependent priors for the smoothing variances were smaller than the ones of inverse gamma priors, MSEs of Scenario 3 are similar for all types of priors. Some sensitivity with respect to $\alpha$ is visible for $\operatorname{IG}(\alpha)$ and $\mathrm{U}(\alpha)$ priors.

(2) Widths of credible intervals. Although MSEs are similar, a slight preference for scale-dependent priors with respect to widths of credible intervals compared to $\operatorname{IG}(\epsilon, \epsilon)$ and flat priors is visible. Similar as for MSEs, $\operatorname{IG}(\alpha)$ and $\mathrm{U}(\alpha)$ are more sensitive with respect to $\alpha$ compared to scale-dependent and half-Cauchy priors.

(3) Coverage rates. Coverage rates of pointwise credible intervals are close to the nominal levels for scale-dependent, $\operatorname{IG}(\epsilon, \epsilon)$, flat and half-normal priors. As already partly found in the previous scenarios, half-normal, half-Cauchy, uniform and $\operatorname{IG}(\alpha)$ priors perform worse as compared to scale-dependent priors. Particularly, the smaller credible intervals of the alternative priors are partly offset by the fact that the corresponding coverage rates are too low for $x \leq-0.5$.

(4) Bias. Approximately unbiased estimates are obtained with a strongly nonlinear effect as is indicated in Figure G25. 


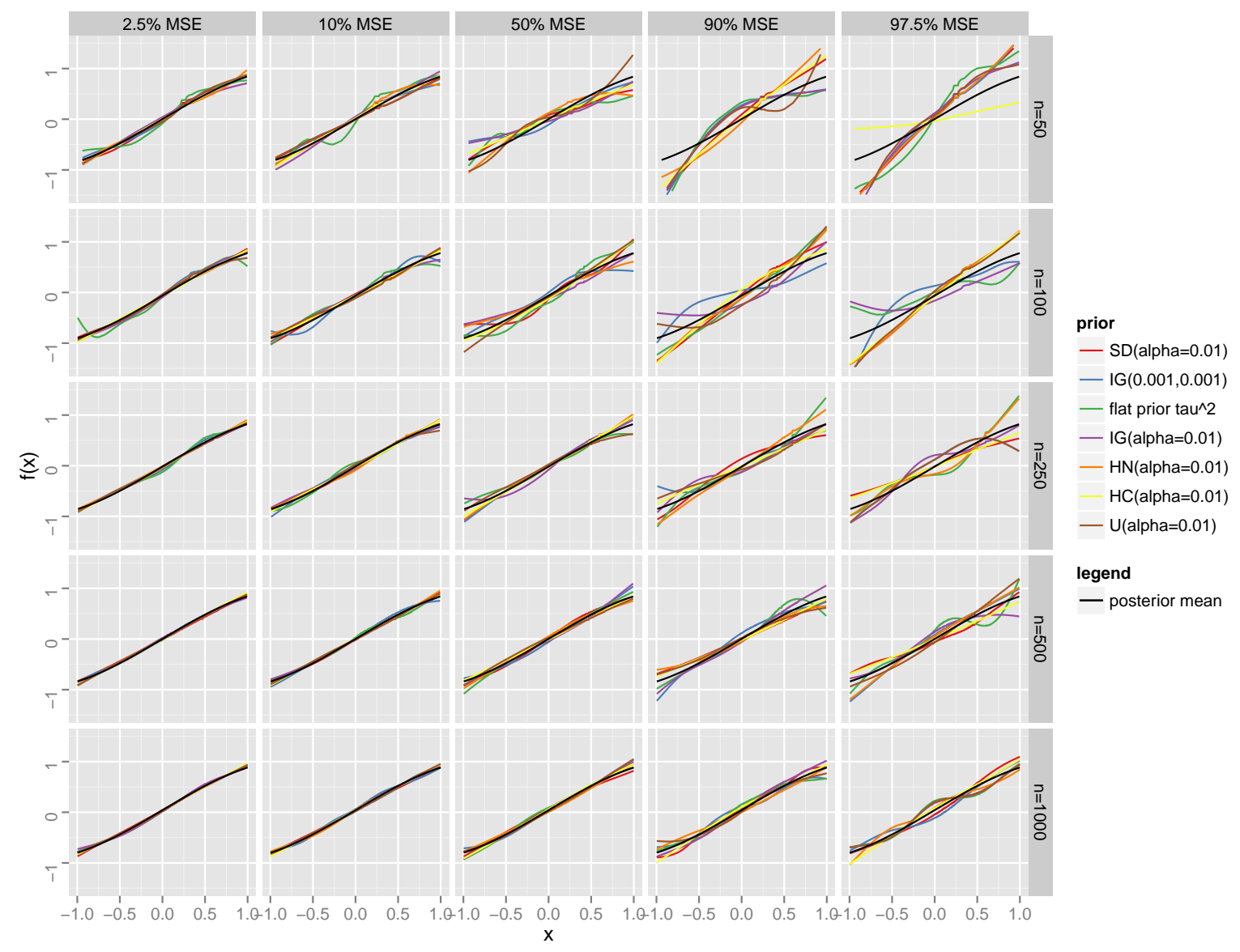

Figure G14: Empirical Evaluation (Scenario 1). Shown are posterior mean estimates for selected replications of different sample sizes $n$ and selected prior settings. The replications are chosen to match the $2.5 \%, 10 \%, 50 \%, 90 \%$ and $97.5 \%$ best MSE of the corresponding 1000 replications. The true function is given by the solid black line.

(5) Selected replications. As expected, Figure G26 reflects the improvement for estimating the true underlying function (plotted with a solid black line) with increasing sample size. Even for small sample sizes, the relatively complicated effect can be estimated reasonably well in all settings. 


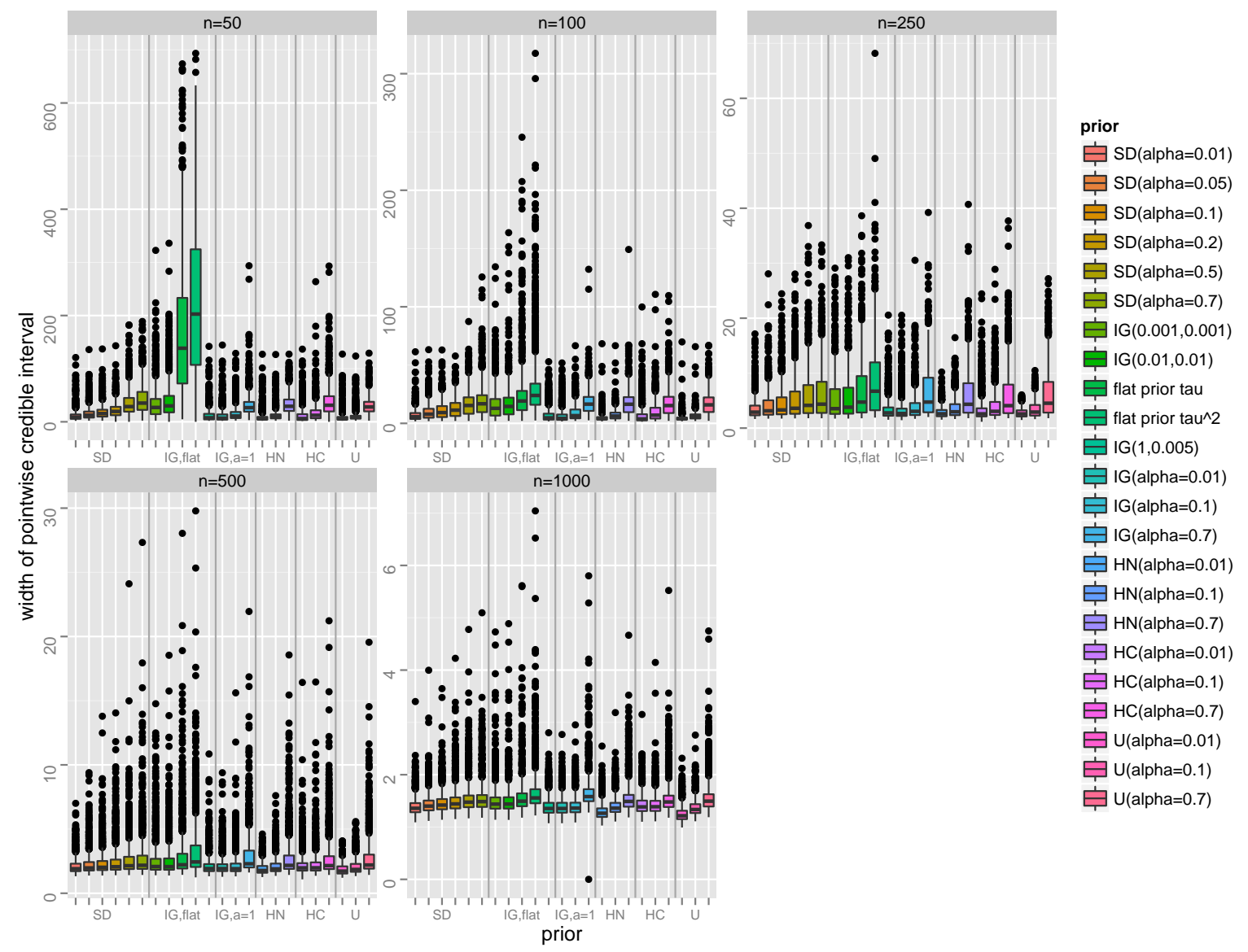

Figure G15: Empirical Evaluation (Scenario 2). Shown are widths of pointwise credible intervals for different sample sizes $n$ and the different prior settings. 


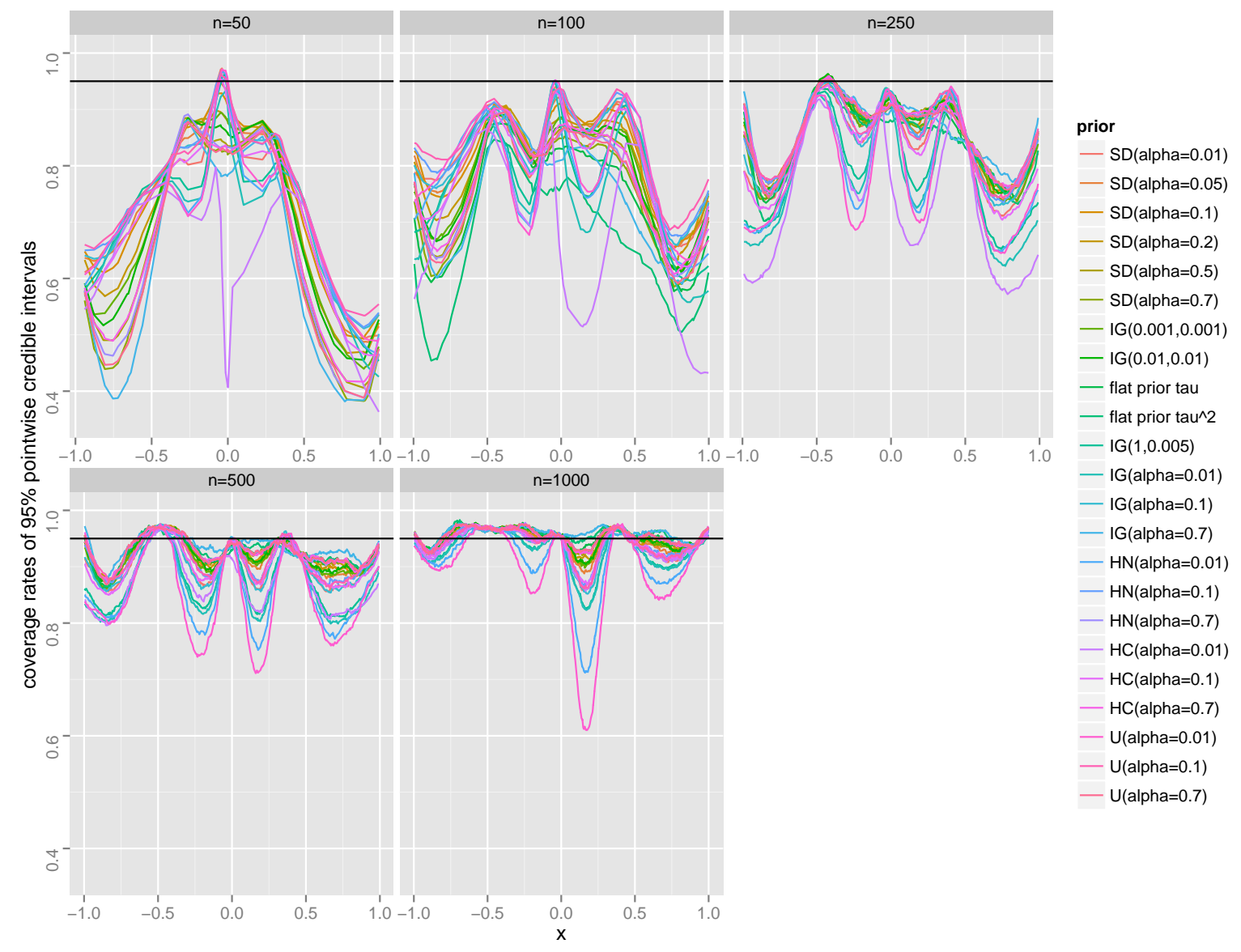

Figure G16: Empirical Evaluation (Scenario 2). Shown are coverage rates of 95\% pointwise credible intervals for different sample sizes $n$ and the different prior settings. 


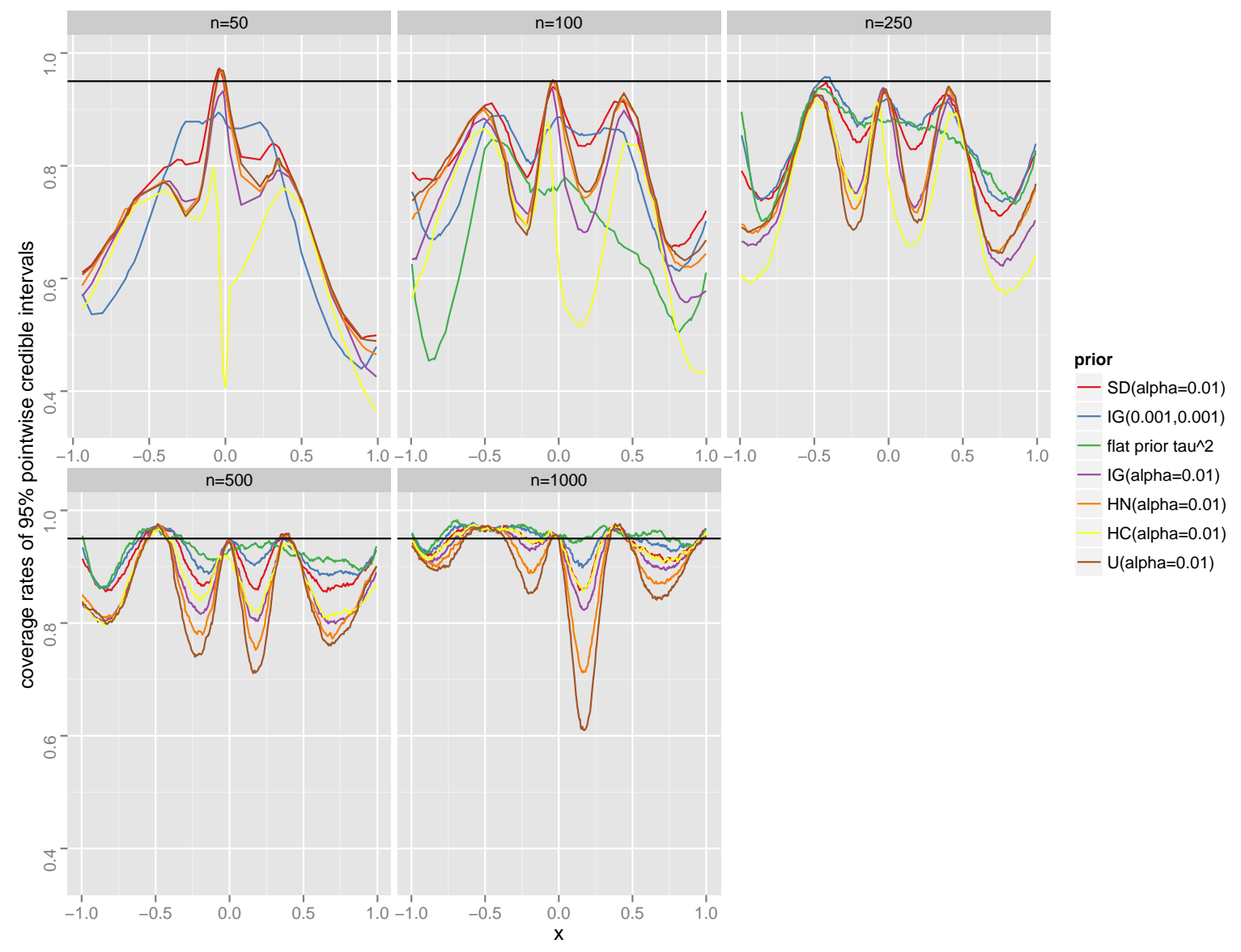

Figure G17: Empirical Evaluation (Scenario 2). Shown are coverage rates of 95\% pointwise credible intervals for different sample sizes $n$ and selected prior settings. 


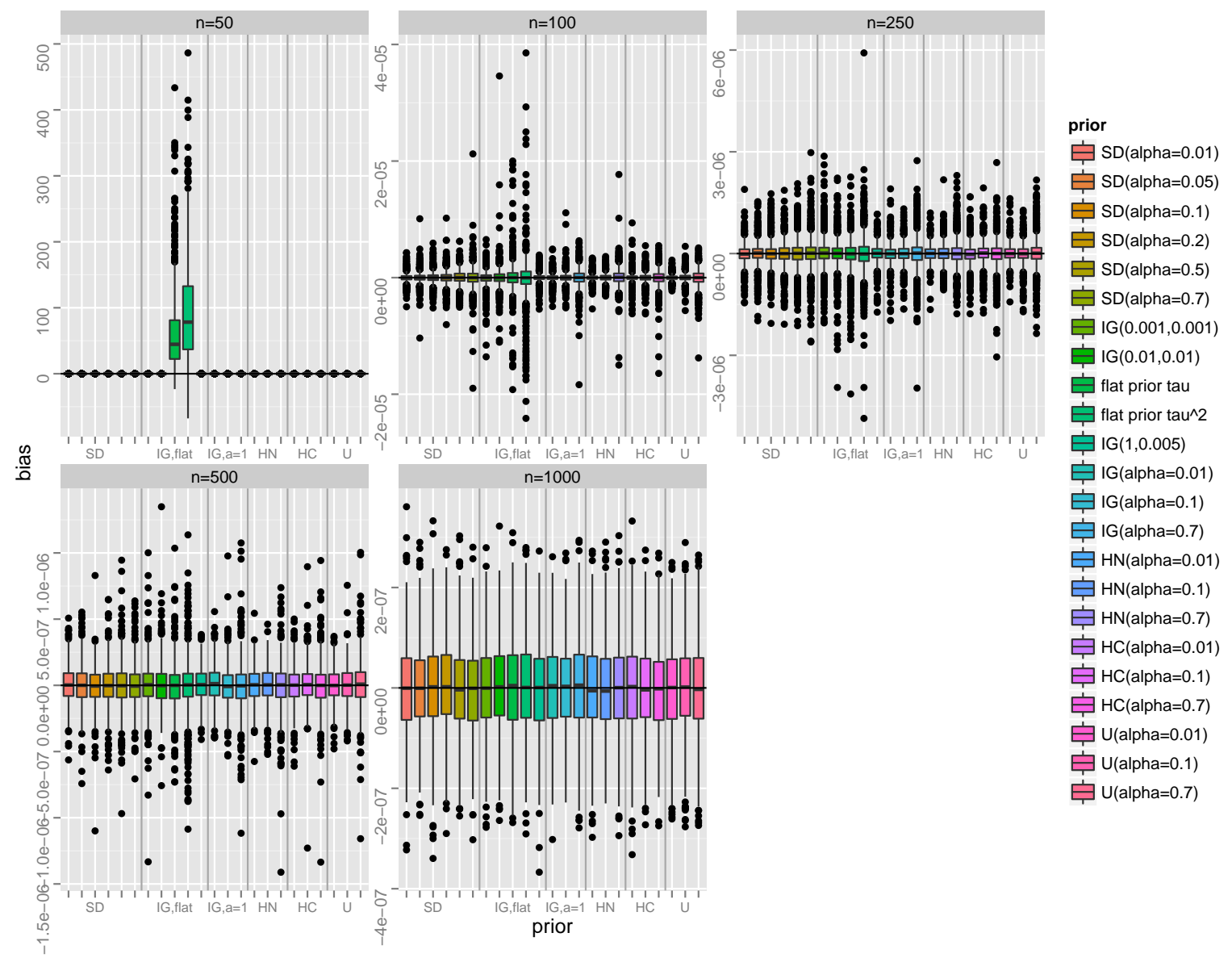

Figure G18: Empirical Evaluation (Scenario 2). Shown are boxplots of bias for different sample sizes $n$ and the different prior settings. 


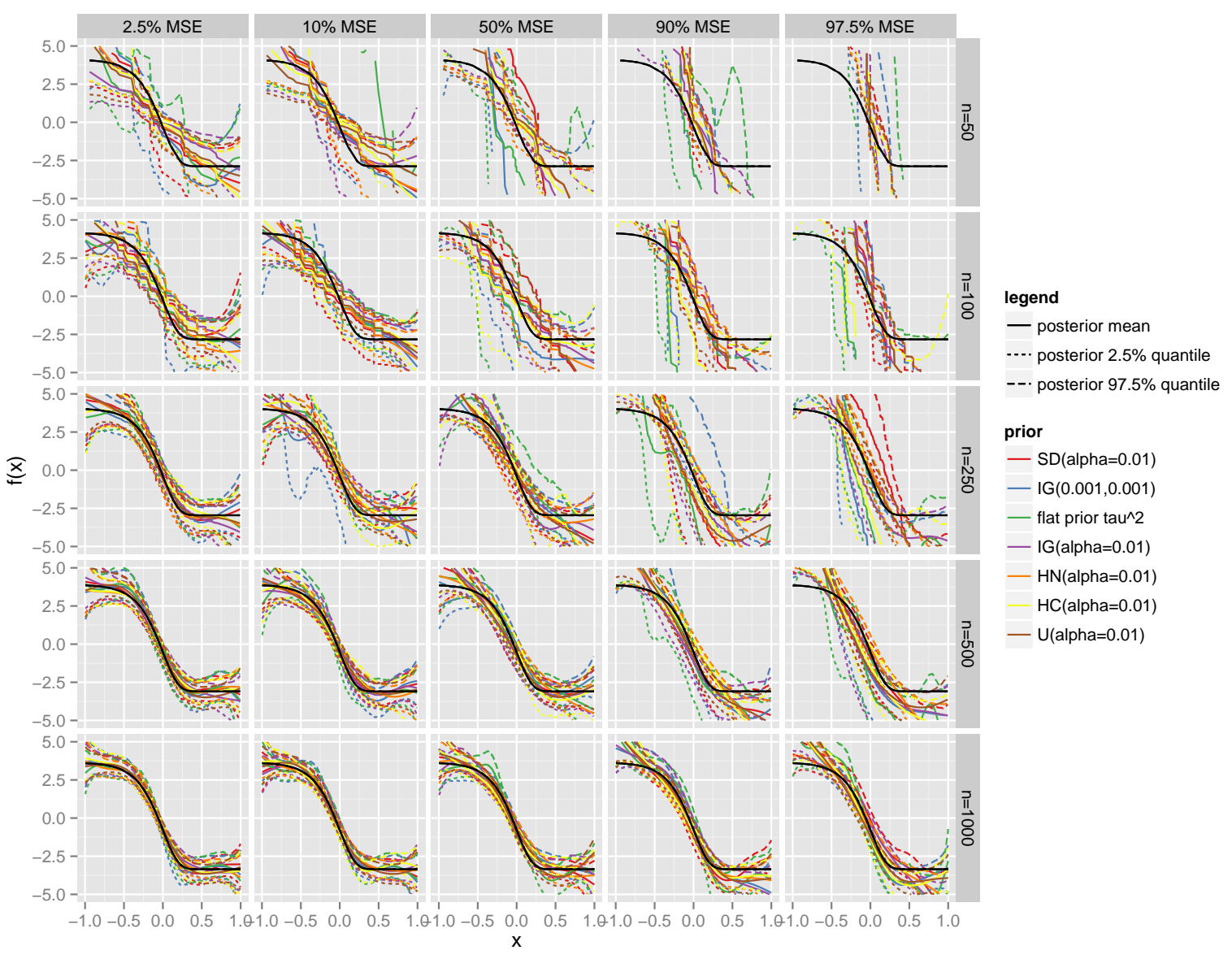

Figure G19: Empirical Evaluation (Scenario 2). Shown are posterior mean, 2.5\% and $97.5 \%$ estimates for selected replications of different sample sizes $n$ and the different prior settings. The replications are chosen to match the $2.5 \%, 10 \%, 50 \%, 90 \%$ and $97.5 \%$ best MSE of the corresponding 1000 replications. The true function is given by the solid black line. 


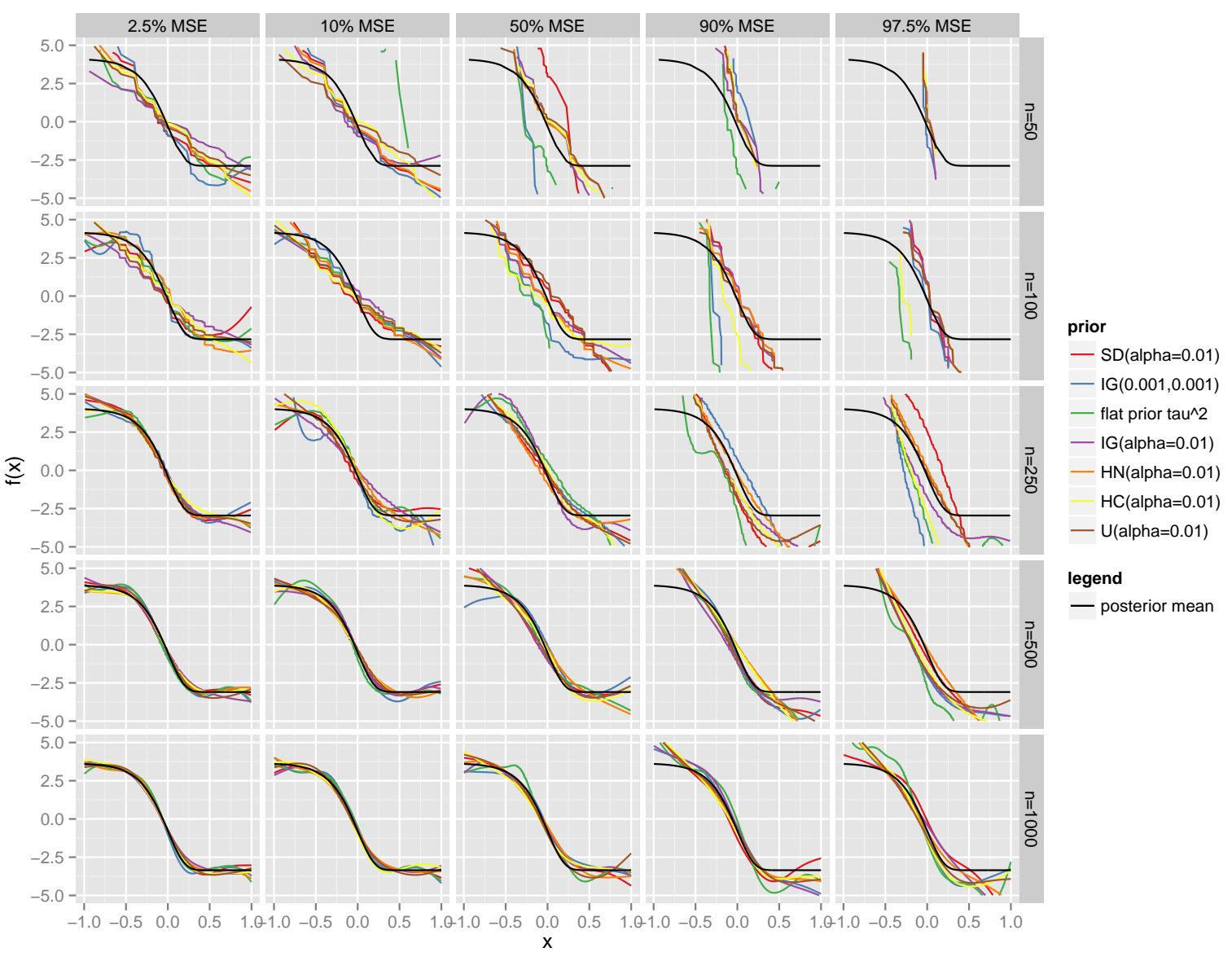

Figure G20: Empirical Evaluation (Scenario 2). Shown are posterior mean estimates for selected replications of different sample sizes $n$ and selected prior settings. The replications are chosen to match the $2.5 \%, 10 \%, 50 \%, 90 \%$ and $97.5 \%$ best MSE of the corresponding 1000 replications. The true function is given by the solid black line. 


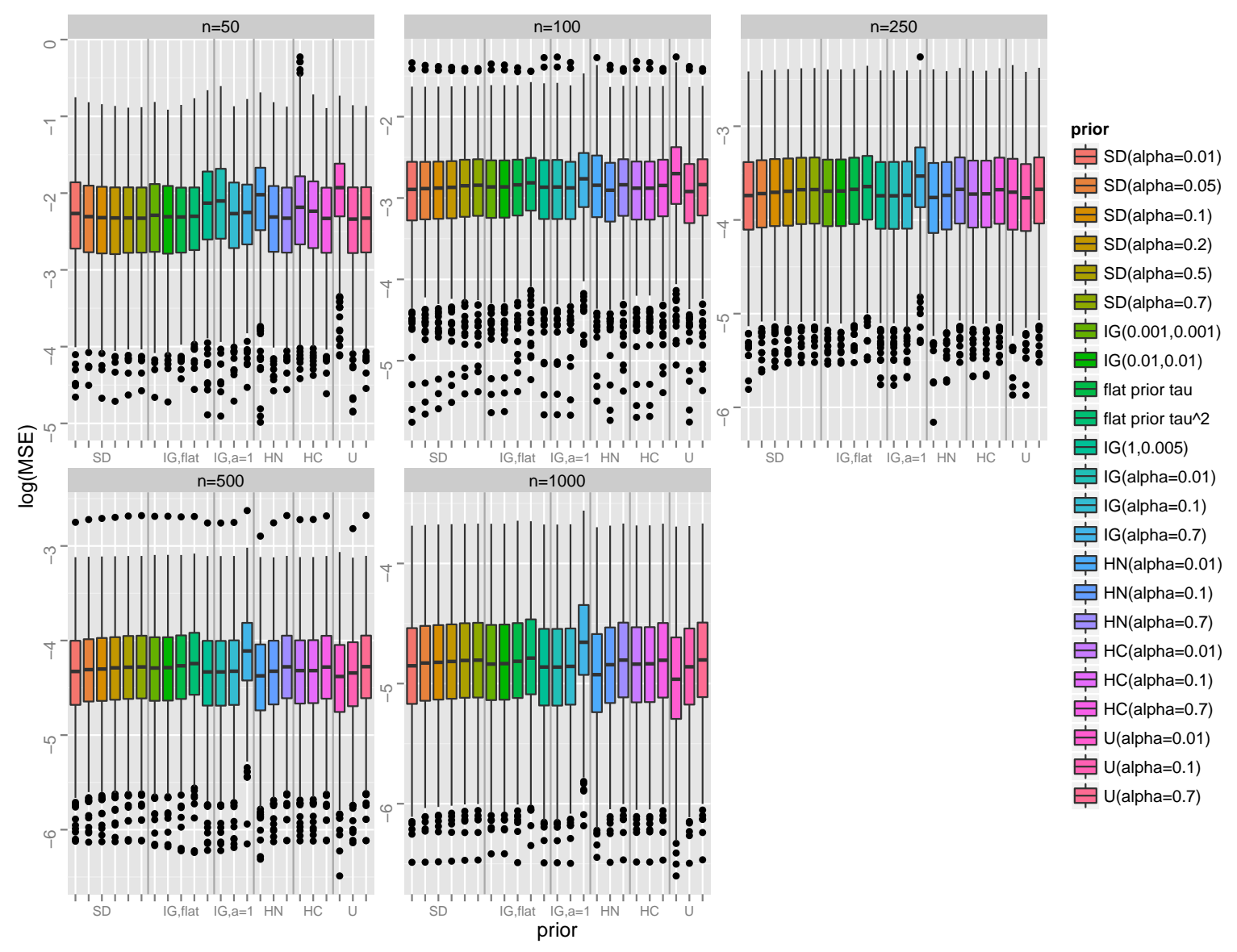

Figure G21: Empirical Evaluation (Scenario 3). Shown are boxplots of log(MSE) for different sample sizes $n$ and the different prior settings. 


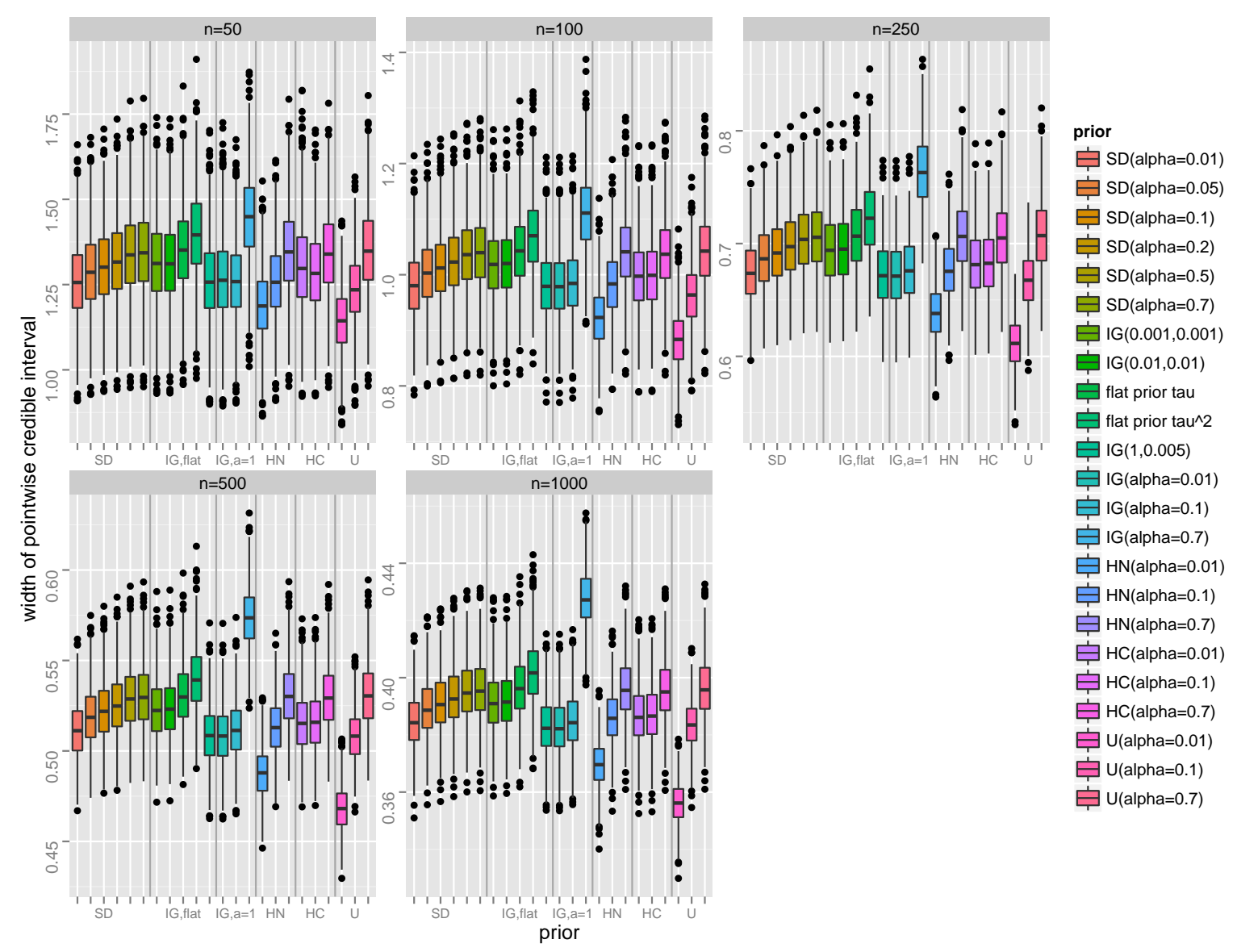

Figure G22: Empirical Evaluation (Scenario 3). Shown are widths of pointwise credible intervals for different sample sizes $n$ and the different prior settings. 


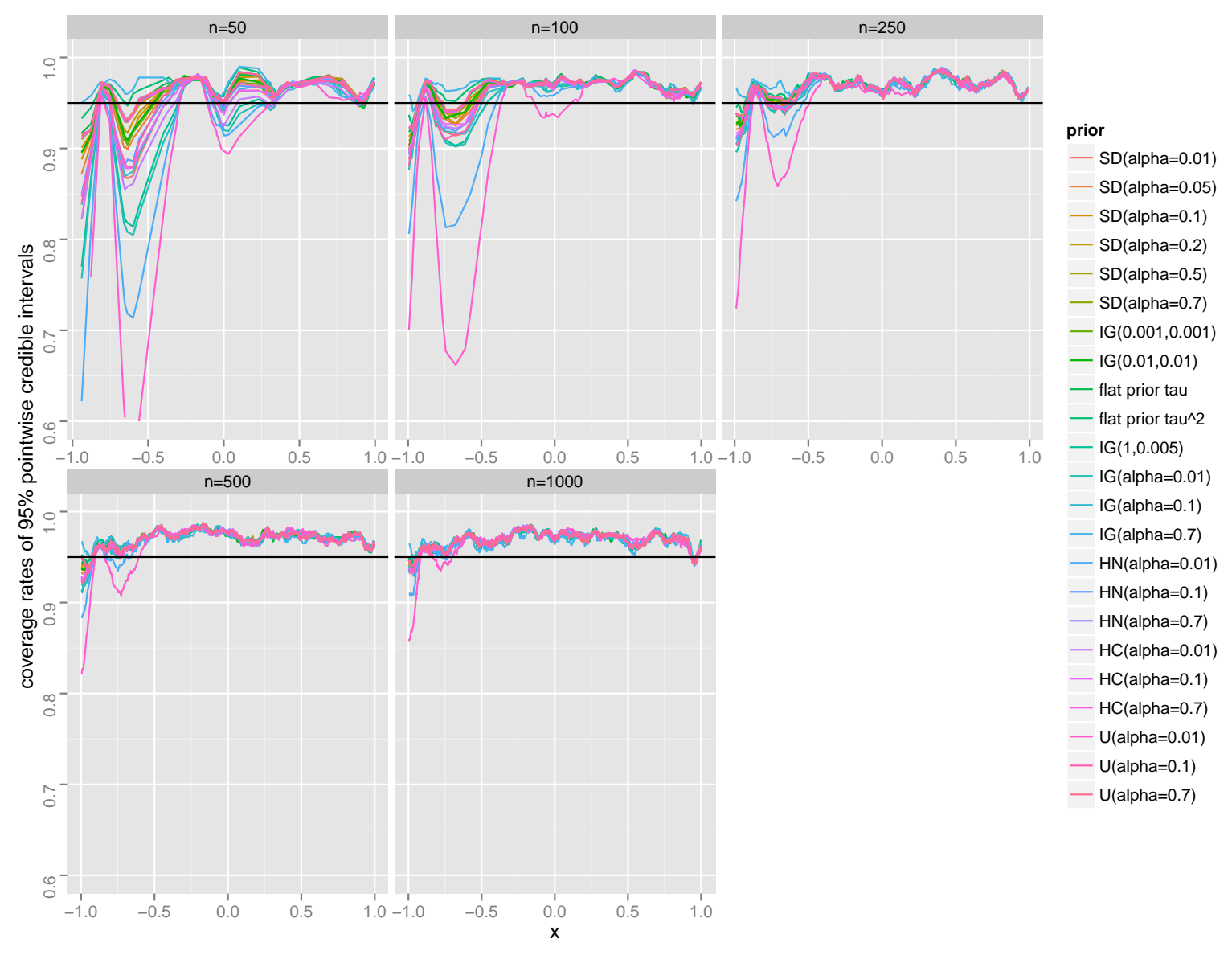

Figure G23: Empirical Evaluation (Scenario 3). Shown are coverage rates of 95\% pointwise credible intervals for different sample sizes $n$ and the different prior settings. 


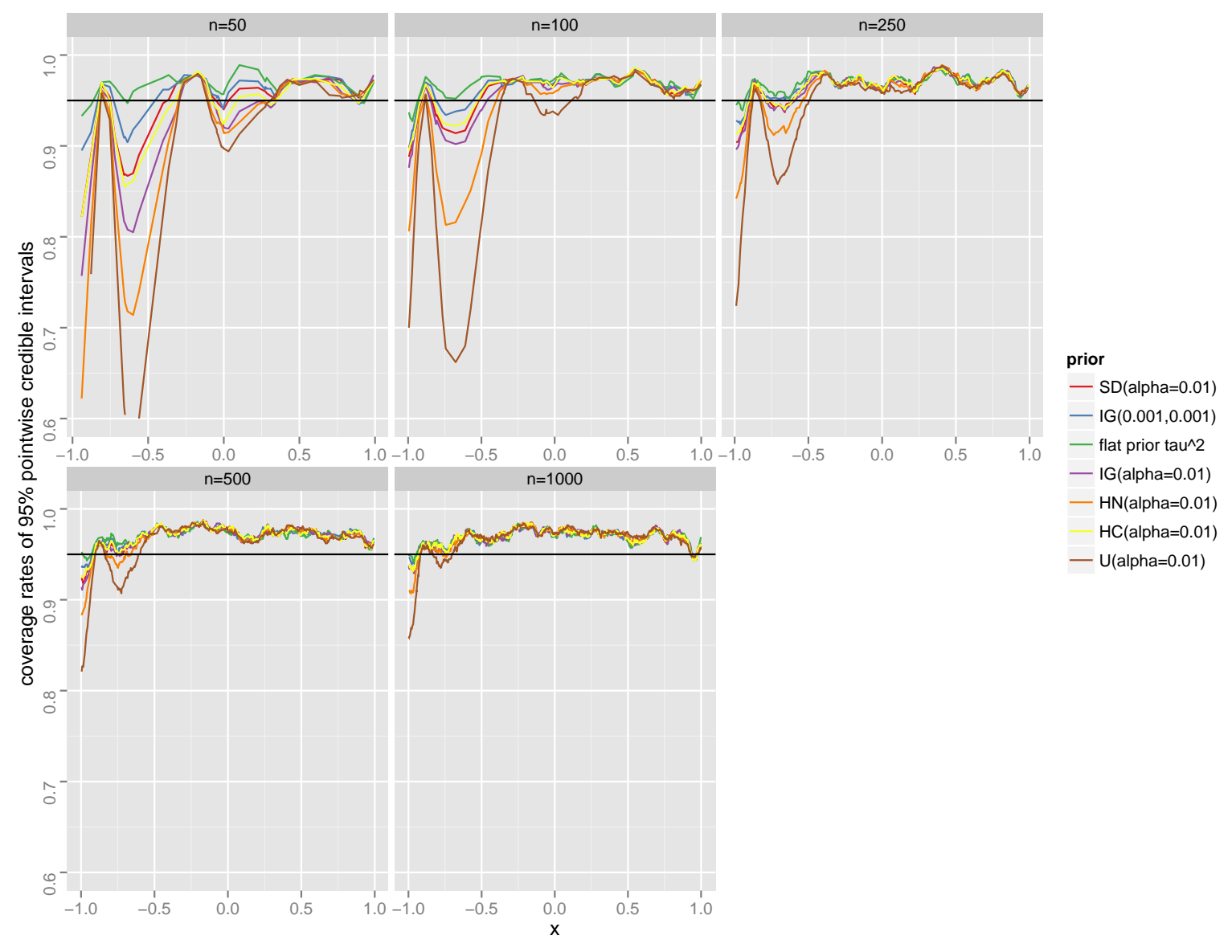

Figure G24: Empirical Evaluation (Scenario 3). Shown are coverage rates of 95\% pointwise credible intervals for different sample sizes $n$ and selected prior settings. 


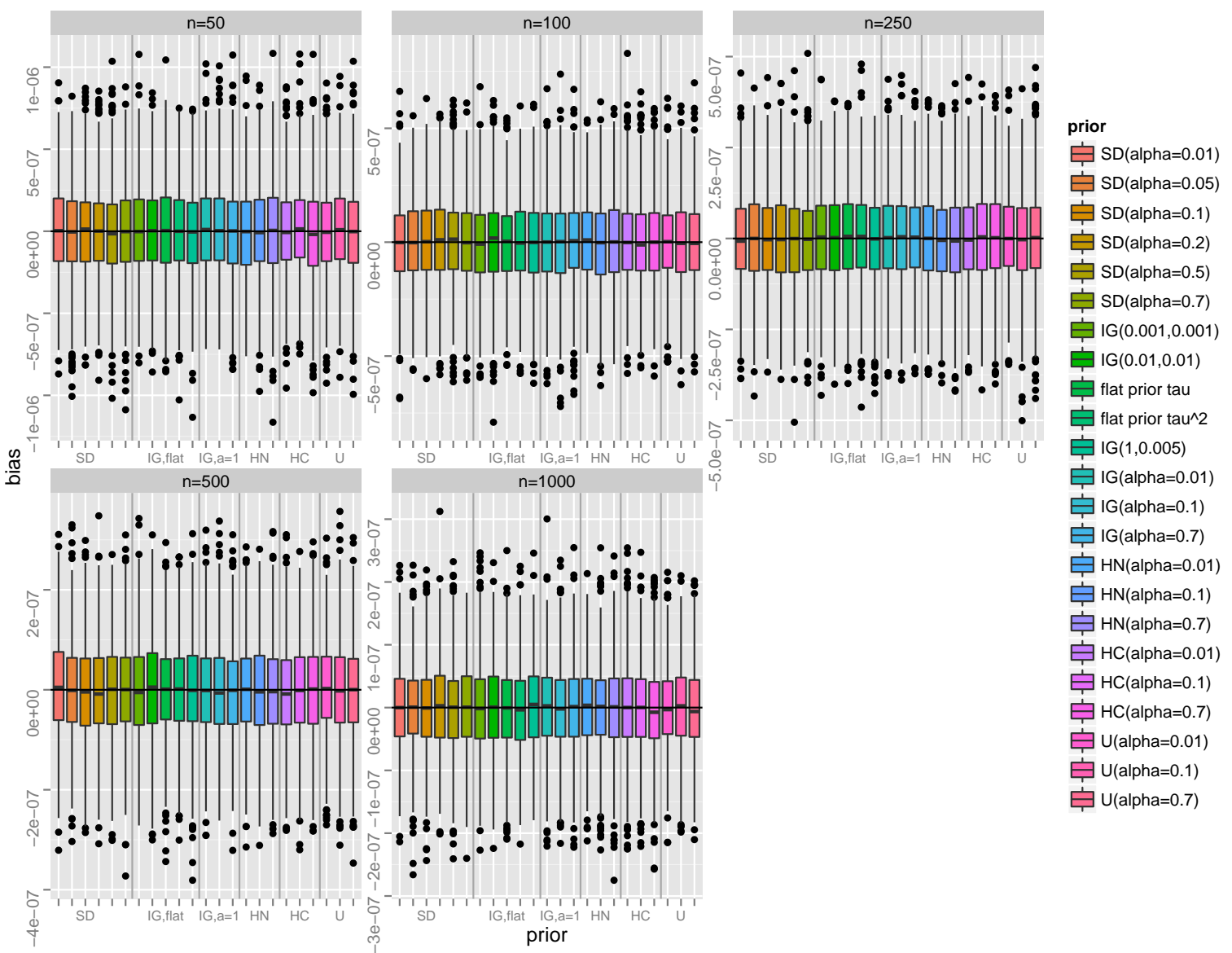

Figure G25: Empirical Evaluation (Scenario 3). Shown are boxplots of bias for different sample sizes $n$ and the different prior settings. 


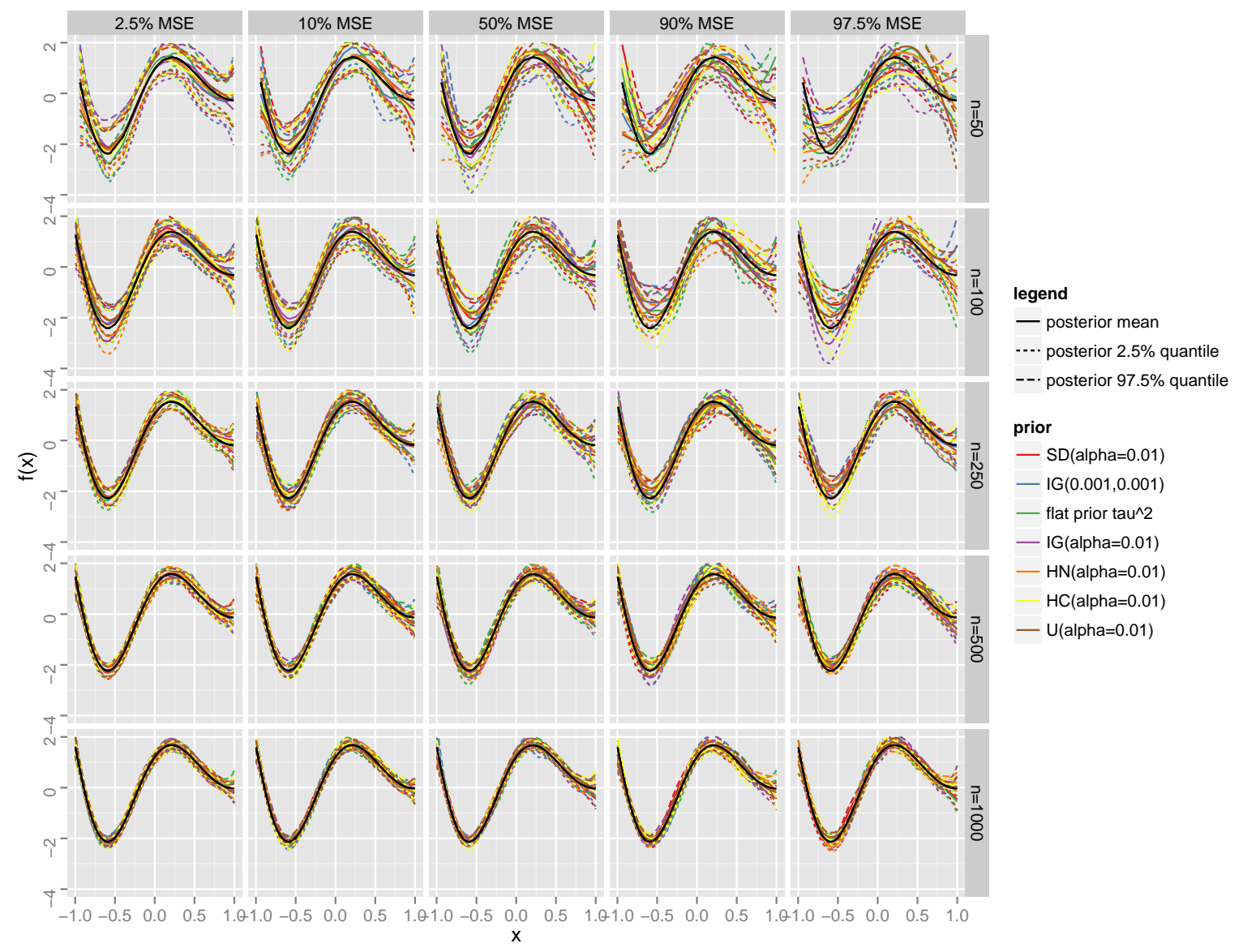

Figure G26: Empirical Evaluation (Scenario 3). Shown are posterior mean, 2.5\% and $97.5 \%$ estimates for selected replications of different sample sizes $n$ and the different prior settings. The replications are chosen to match the $2.5 \%, 10 \%, 50 \%, 90 \%$ and $97.5 \%$ best MSE of the corresponding 1000 replications. The true function is given by the solid black line. 


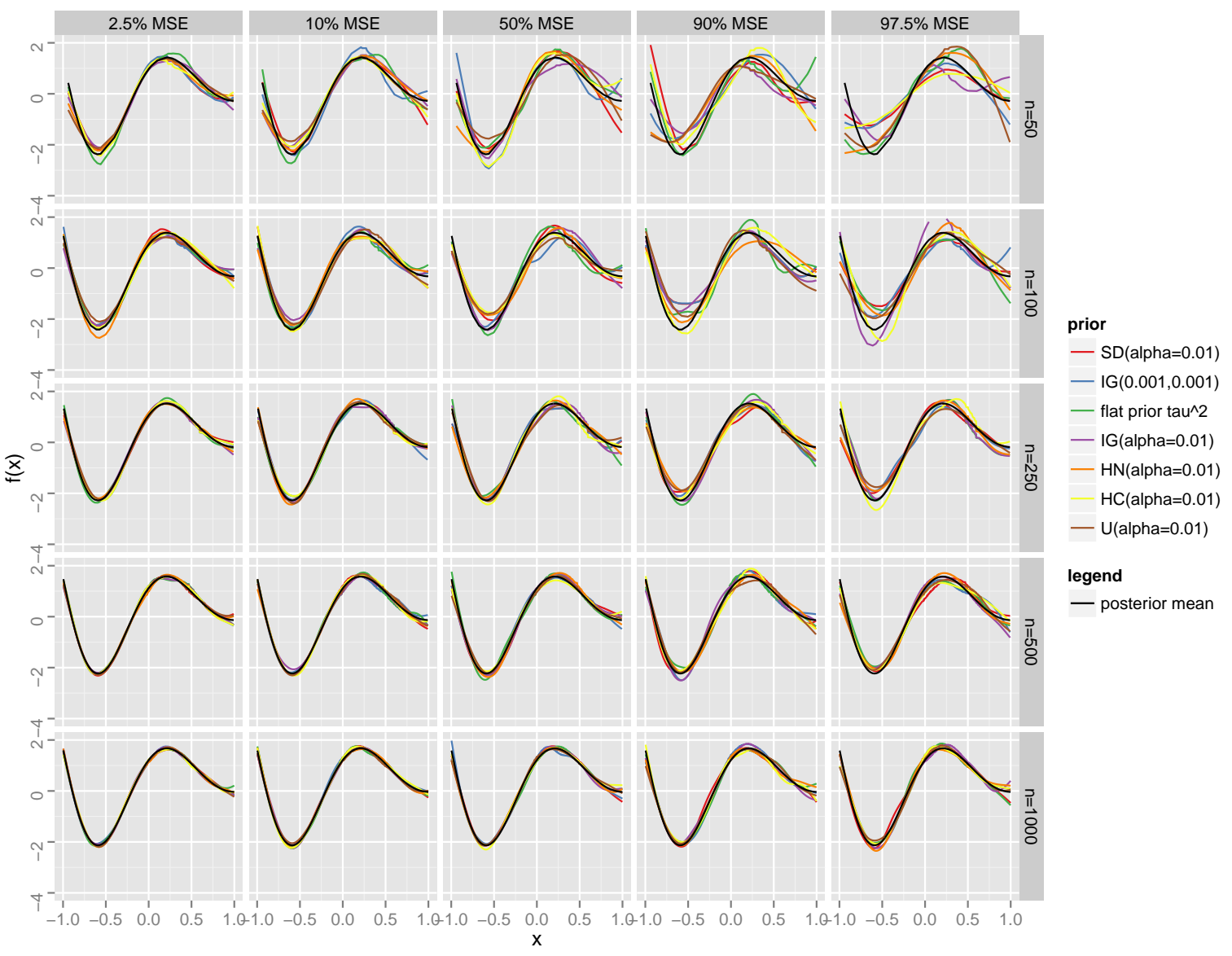

Figure G27: Empirical Evaluation (Scenario 3). Shown are posterior mean estimates for selected replications of different sample sizes $n$ and selected prior settings. The replications are chosen to match the $2.5 \%, 10 \%, 50 \%, 90 \%$ and $97.5 \%$ best MSE of the corresponding 1000 replications. The true function is given by the solid black line. 


\section{H Patent Citations}
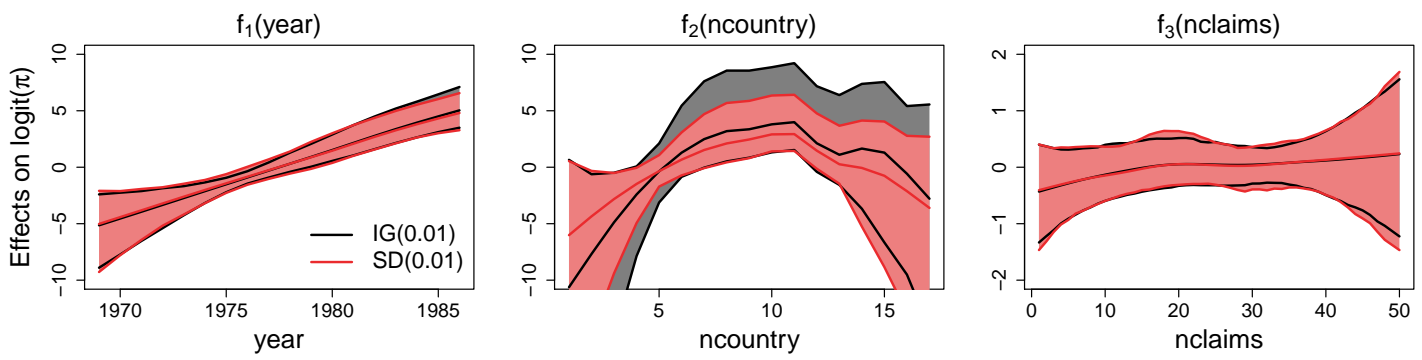

Figure H28: Patent citations. Comparison of estimated nonlinear effects of continuous covariates year, ncountry and nclaims on logit $(\pi)$. Shown are posterior means and $95 \%$ credible intervals with scale-dependent priors (red) and inverse gamma priors, $a=1$ (black), $\alpha=0.01, c=3$.
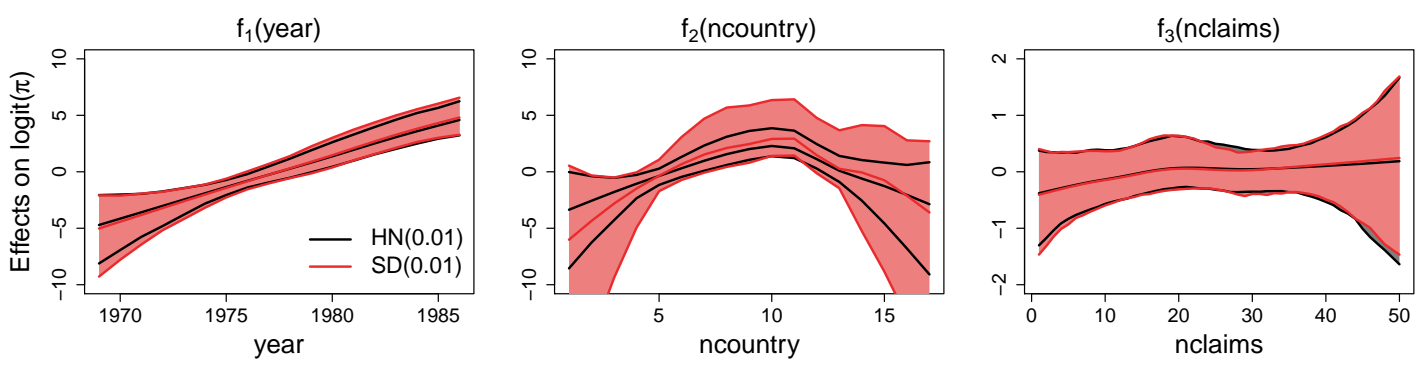

Figure H29: Patent citations. Comparison of estimated nonlinear effects of continuous covariates year, ncountry and nclaims on logit $(\pi)$. Shown are posterior means and $95 \%$ credible intervals with scale-dependent priors (red) and half-normal priors for $\tau$ (black), $\alpha=0.01, c=3$.
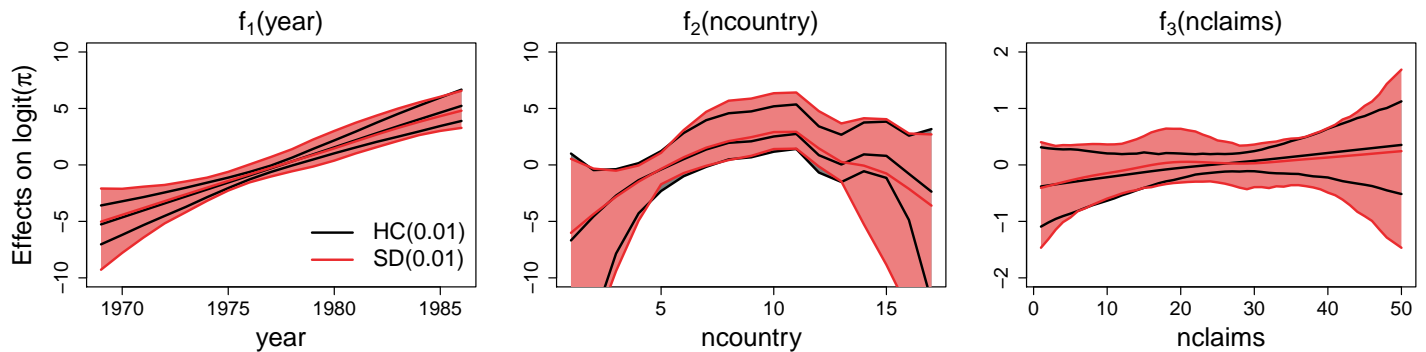

Figure H30: Patent citations. Comparison of estimated nonlinear effects of continuous covariates year, ncountry and nclaims on logit $(\pi)$. Shown are posterior means and $95 \%$ credible intervals with scale-dependent priors (red) and half-Cauchy priors for $\tau$ (black), $\alpha=0.01, c=3$.
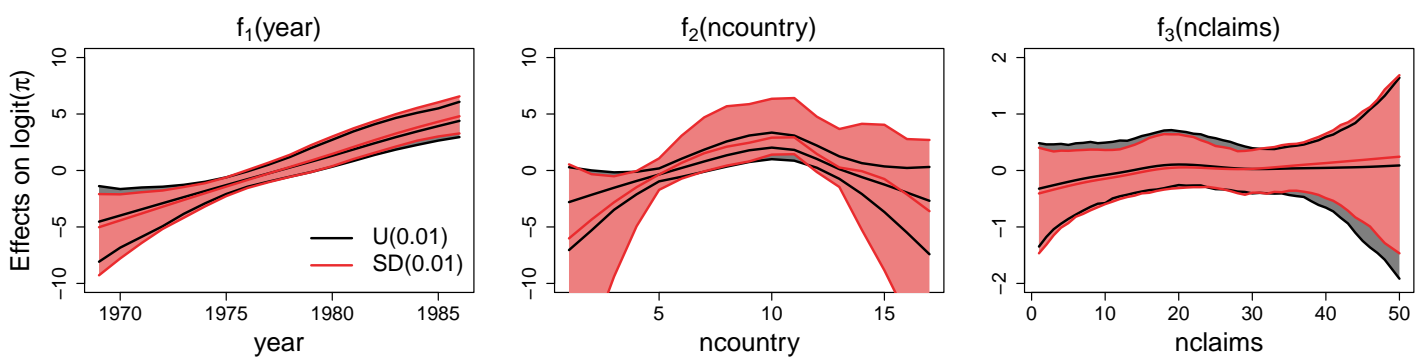

Figure H31: Patent citations. Comparison of estimated nonlinear effects of continuous covariates year, ncountry and nclaims on logit $(\pi)$. Shown are posterior means and $95 \%$ credible intervals with scale-dependent priors (red) and approximate uniform priors for $\tau$ (black), $\alpha=0.01, c=3$. 


\section{Childhood Undernutrition in Zambia}
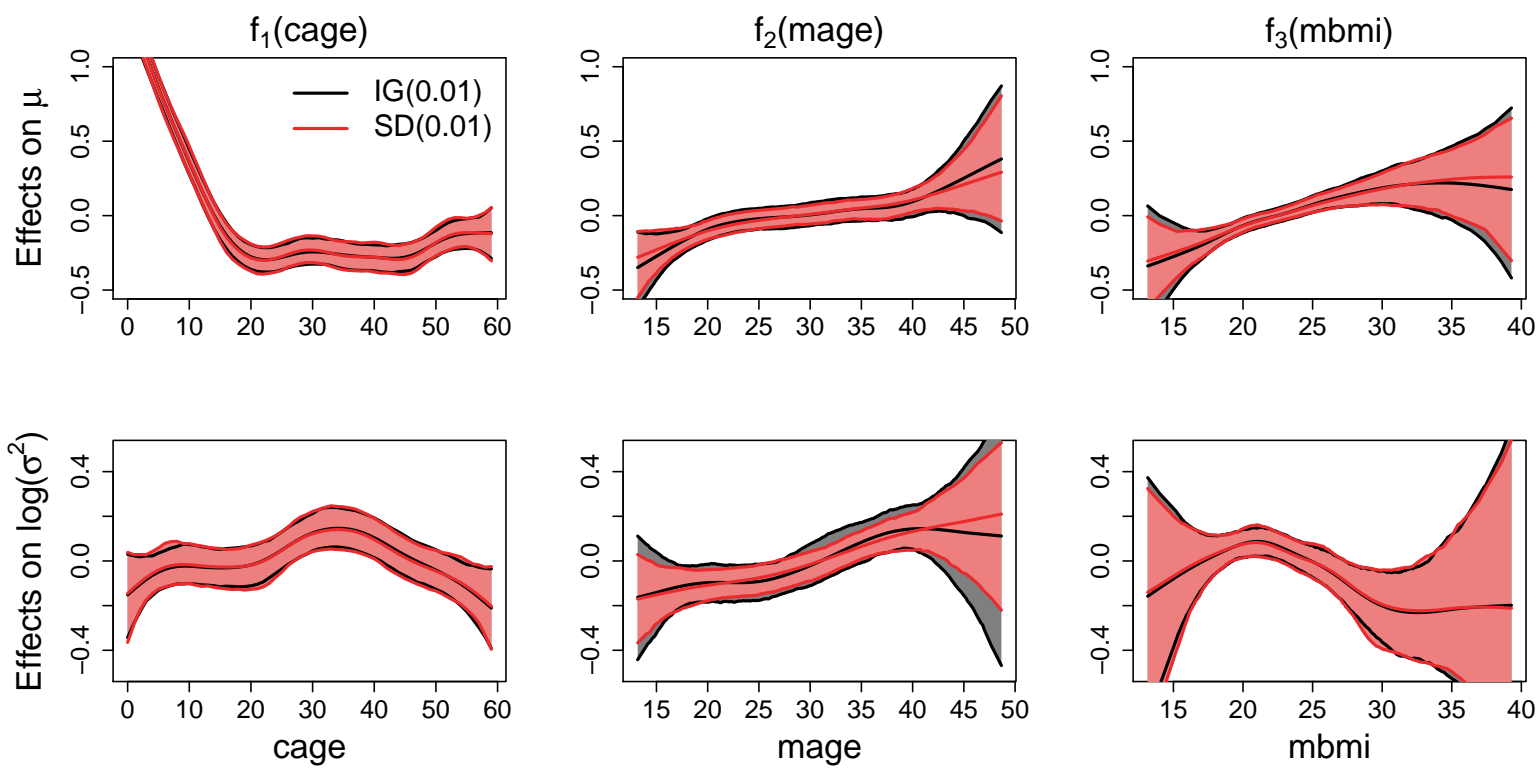

Figure I32: Childhood undernutrition. Comparison of estimated nonlinear effects of continuous covariates cage, mage and mbmi with scale-dependent priors (red) and inverse gamma priors, $a=1$ (black), $\alpha=0.01, c=3$. Shown are posterior means and $95 \%$ credible intervals on $\mathbb{E}($ zscore $)$ (top) and on $\log \left(\sigma^{2}\right)$ (bottom).
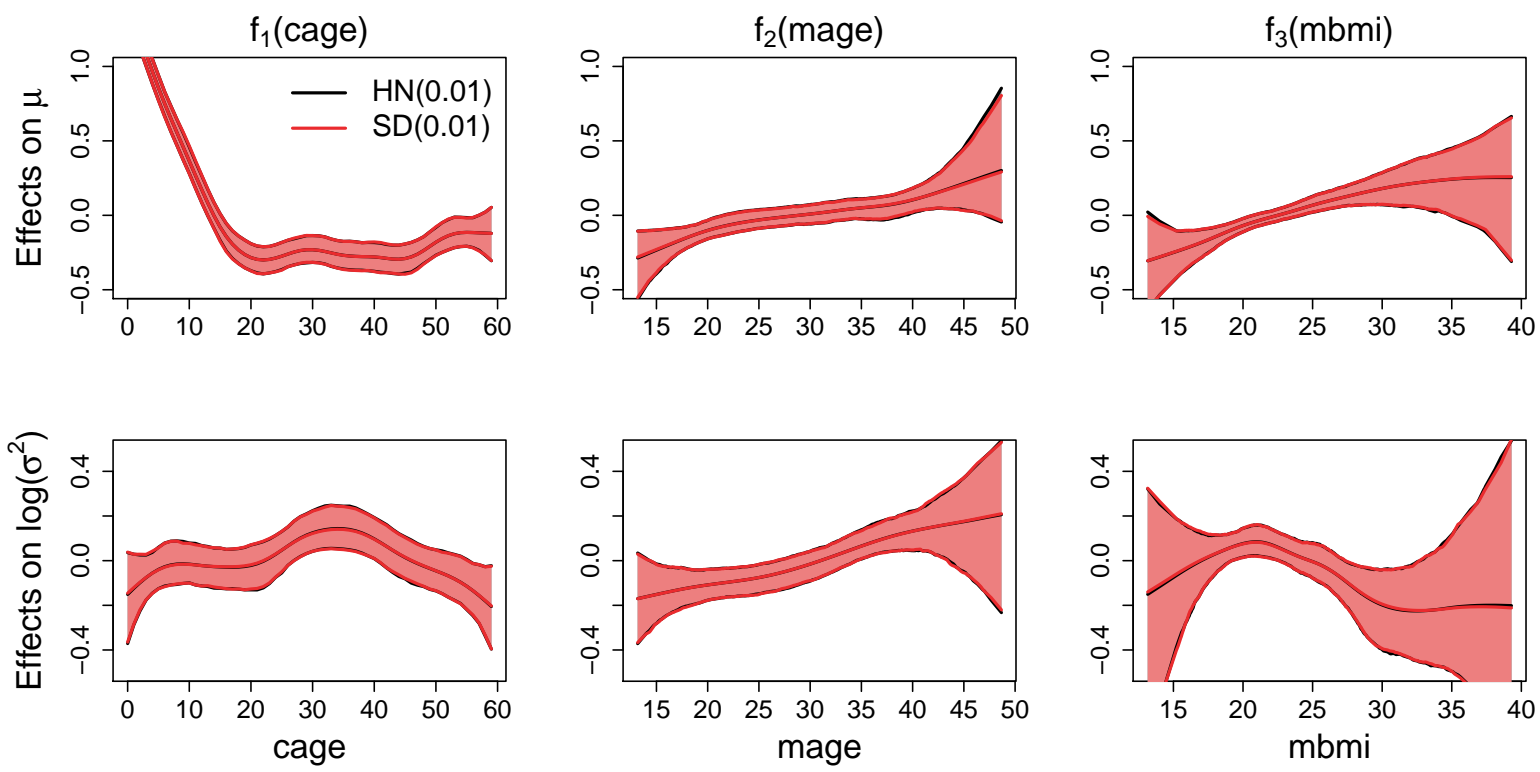

Figure I33: Childhood undernutrition. Comparison of estimated nonlinear effects of continuous covariates cage, mage and mbmi with scale-dependent priors (red) and half-normal priors for $\tau$ (black), $\alpha=0.01, c=3$. Shown are posterior means and $95 \%$ credible intervals on $\mathbb{E}$ (zscore) (top) and on $\log \left(\sigma^{2}\right)$ (bottom). 

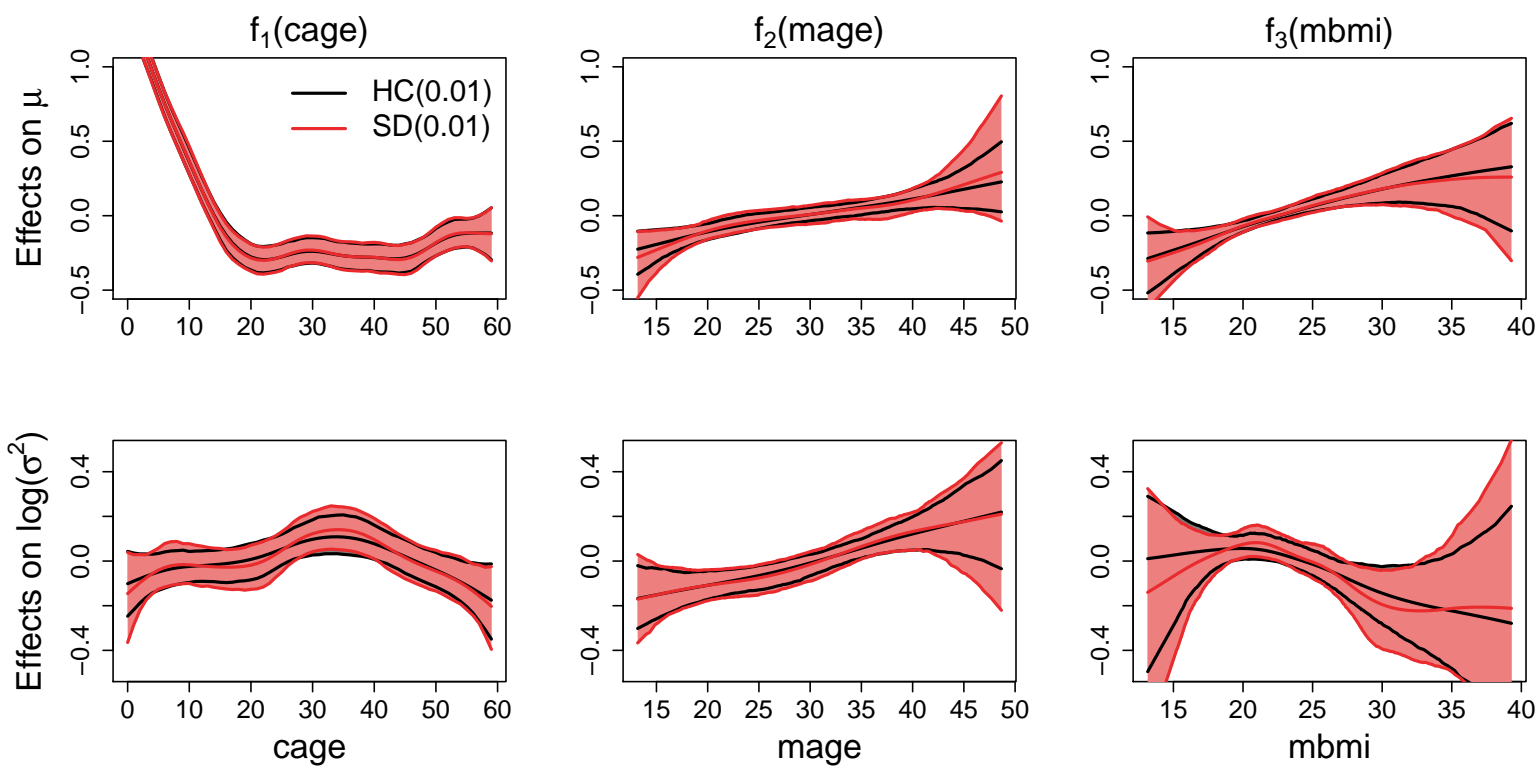

Figure I34: Childhood undernutrition. Comparison of estimated nonlinear effects of continuous covariates cage, mage and mbmi with scale-dependent priors (red) and half-Cauchy priors for $\tau$ (black), $\alpha=0.01, c=1$. Shown are posterior means and $95 \%$ credible intervals on $\mathbb{E}($ zscore) (top) and on $\log \left(\sigma^{2}\right)$ (bottom).
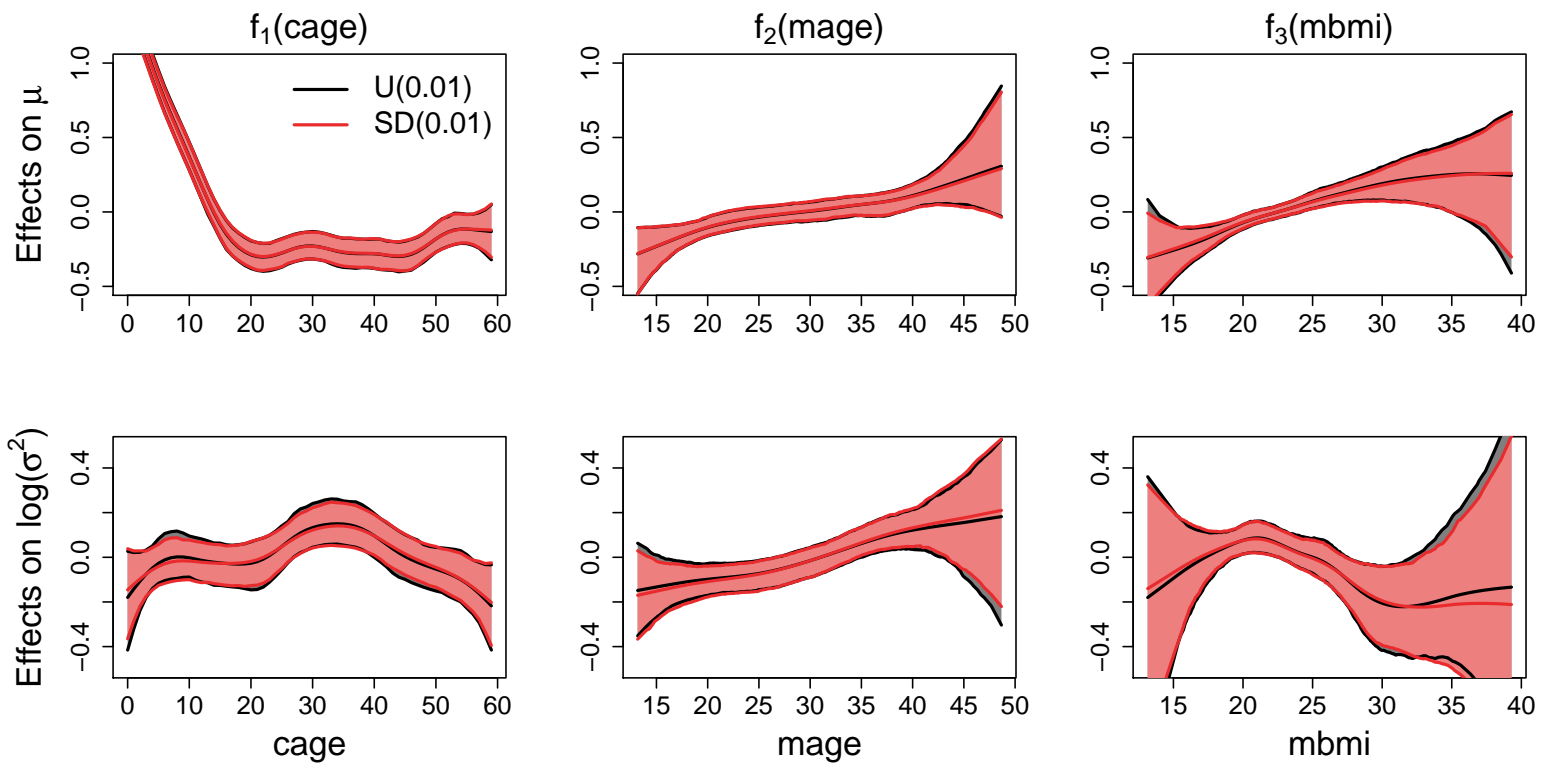

Figure I35: Childhood undernutrition. Comparison of estimated nonlinear effects of continuous covariates cage, mage and mbmi with scale-dependent priors (red) and approximate uniform priors for $\tau$ (black), $\alpha=0.01, c=3$. Shown are posterior means and $95 \%$ credible intervals on $\mathbb{E}($ zscore $)$ (top) and on $\log \left(\sigma^{2}\right)$ (bottom). 


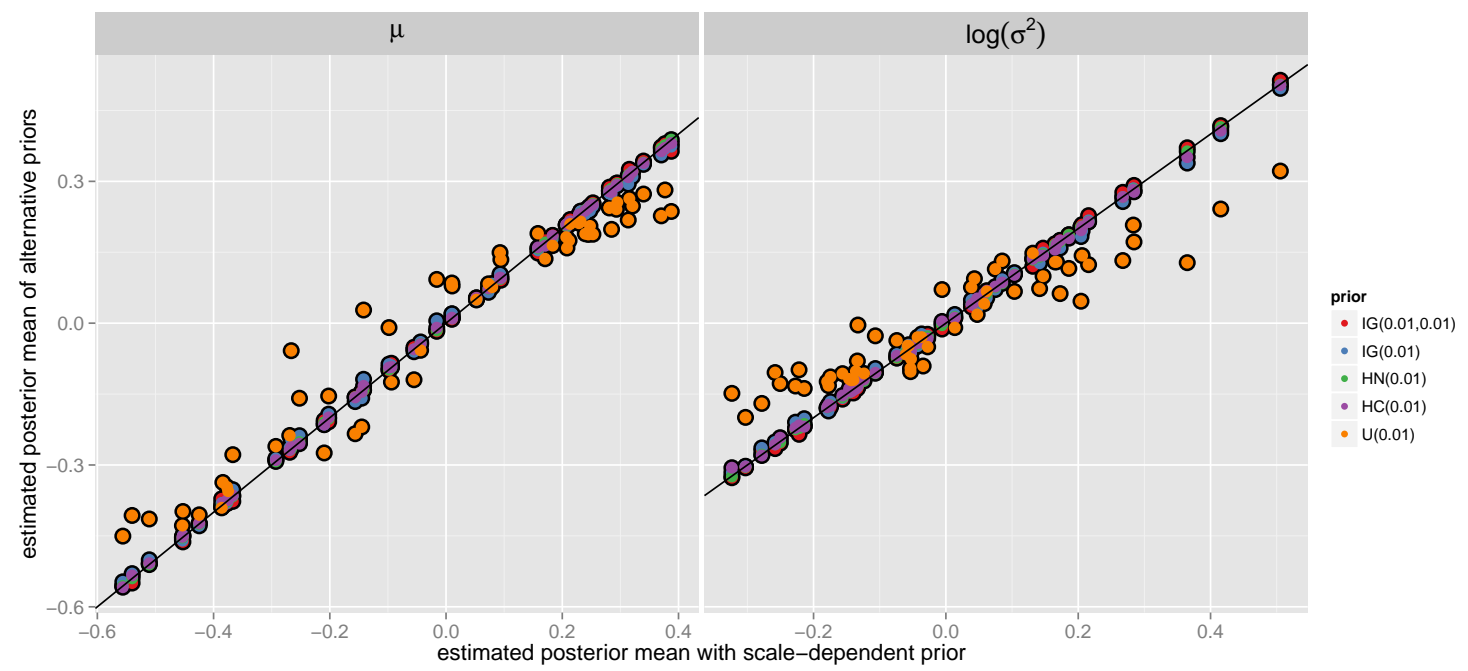

Figure I36: Childhood undernutrition. Comparison of estimated spatial effects with alternative priors (y-axis) and scale-dependent priors (x-axis), $\alpha=0.01, c=3$. Shown are posterior estimates on $\mathbb{E}($ zscore $)$ (left) and on $\log \left(\sigma^{2}\right)$ (right).

Spatial effect on $\mu, I G(0.01)$

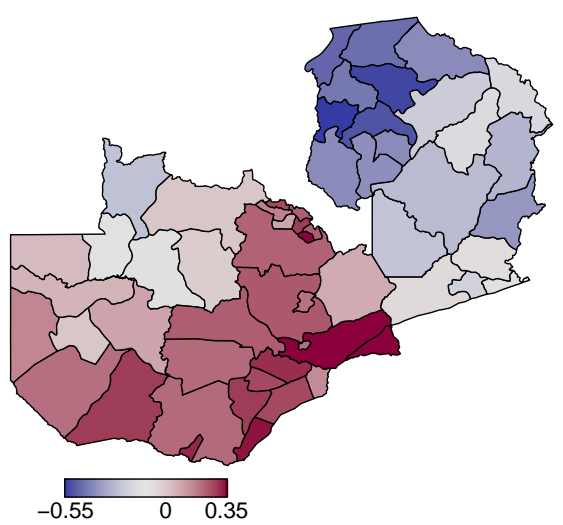

Spatial effect on $\log \left(\sigma^{2}\right), I G(0.01)$

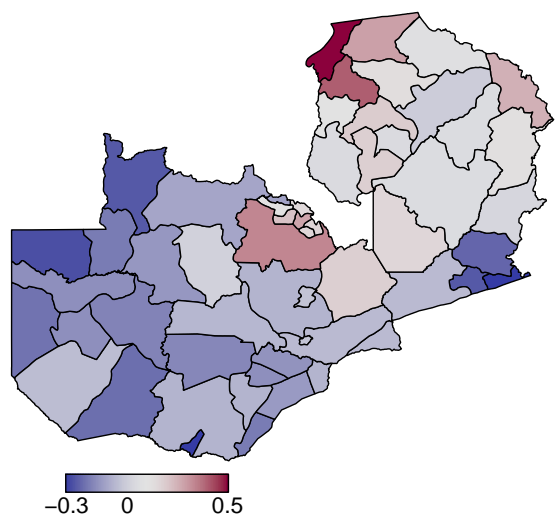

Spatial effect on $\mu, \mathrm{HN}(0.01)$

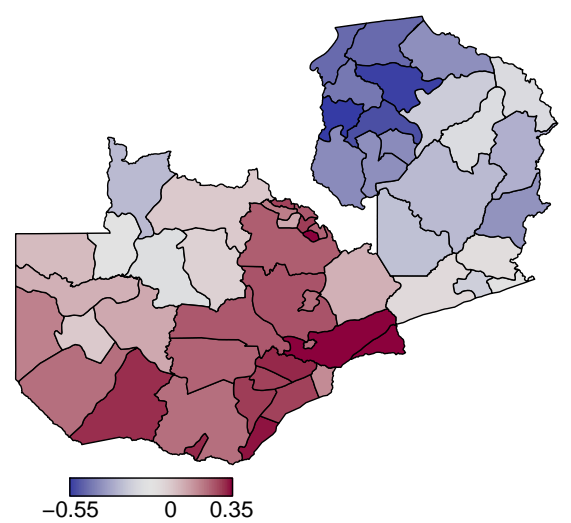

Spatial effect on $\log \left(\sigma^{2}\right), \mathrm{HN}(0.01)$

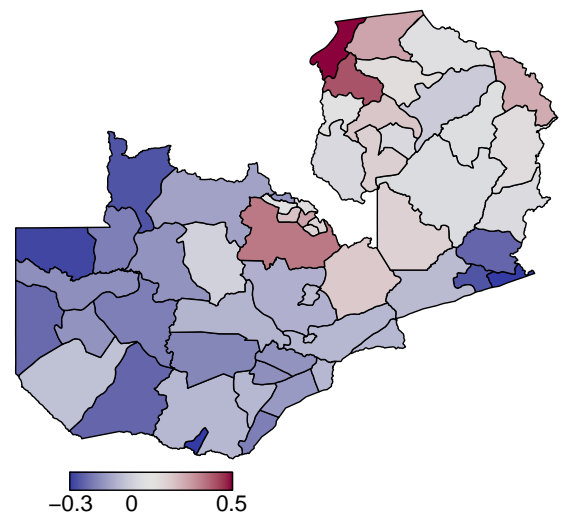

Figure I37: Childhood undernutrition. Comparison of estimated spatial effects with inverse gamma priors, $a=1$ (left) and half-normal priors (right), $\alpha=0.01, c=3$. Shown are posterior estimates on $\mathbb{E}($ zscore $)$ (top) and on $\log \left(\sigma^{2}\right)$ (bottom). 
Spatial effect on $\mu, \mathrm{HC}(0.01)$

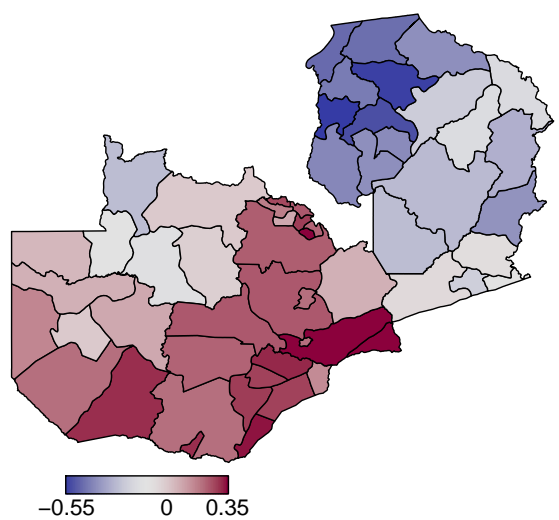

Spatial effect on $\log \left(\sigma^{2}\right), \mathrm{HC}(0.01)$

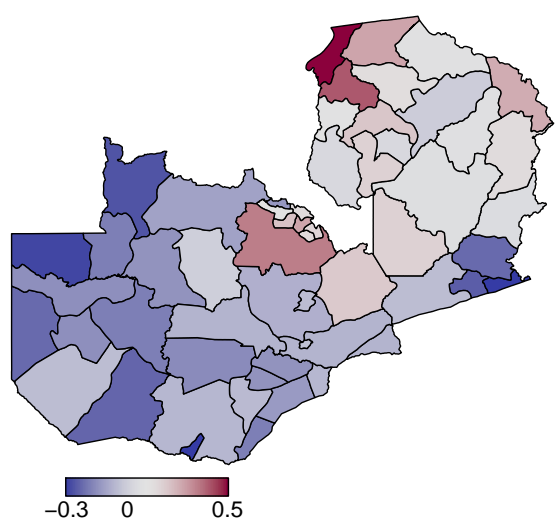

Spatial effect on $\mu, U(0.01)$

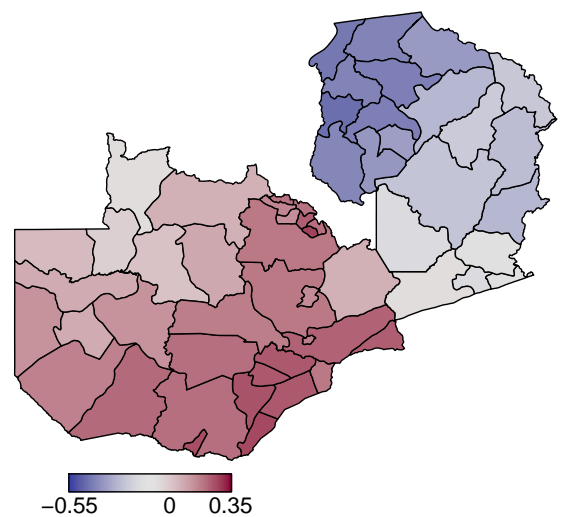

Spatial effect on $\log \left(\sigma^{2}\right), U(0.01)$

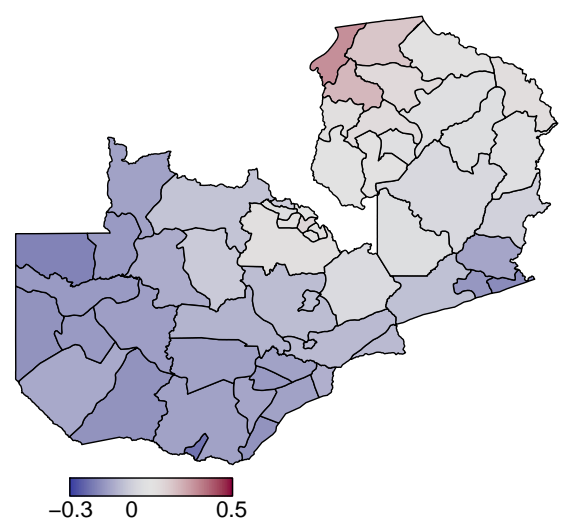

Figure I38: Childhood undernutrition. Comparison of estimated spatial effects with half-Cauchy priors for $\tau$ (left) and approximate uniform priors (right), $\alpha=0.01, c=3$ (right). Shown are posterior estimates on $\mathbb{E}$ (zscore) (top) and on $\log \left(\sigma^{2}\right)$ (bottom). 


\section{References}

Klein, N., Kneib, T. and Lang, S. (2015). Bayesian generalized additive models for location, scale and shape for zero-inflated and overdispersed count data, Journal of the American Statistical Association 110: 405-419. doi:10.1080/01621459.2014.912955.

Sun, D., Tsutakawa, R. K. and He, Z. (2001). Propriety of posteriors with improper priors in hierarchical linear mixed models, Statistica Sinica 11: 77-95. 